Análise empírica de dados multinomiais 

SERVIÇO DE PÓS-GRADUAÇÃO DO ICMC-USP

Data de Depósito:

Assinatura:

\title{
Análise empírica de dados multinomiais ${ }^{1}$
}

\author{
Renata Pelissari
}

Orientador: Prof. Dr. Dorival Leão P. Júnior

Dissertação apresentada ao Instituto de Ciências Matemáticas e de Computação - ICMC-USP, como parte dos requisitos para obtenção do título de Mestre em Ciências - Ciências de Computação e Matemática Computacional.

\author{
USP - São Carlos \\ Julho/2009
}

\footnotetext{
${ }^{1}$ Este trabalho contou com o apoio financeiro do CNPq e da Fapesp.
} 

Aos meus pais,

Antonio e Sandra com amor ... 



\section{Agradecimentos}

Agradeço a Deus por me capacitar e me dar força para concluir mais essa etapa na minha vida.

Aos meus pais, Antonio Pelissari e Sandra Barbosa Pelissari, pelo esforço, dedicação, apoio incondicional e educação. Sempre priorizaram meus estudos, independente das circunstâncias, e graças a eles cheguei até aqui. À minha irmã Fernanda e ao meu cunhado Rafael, pela ajuda em todos as áreas da minha vida. Amo vocês!

Ao Prof. Dorival Leão, pela orientação deste trabalho, pela amizade e disponibilidade, sempre quando precisei. Agradeço também pelo conhecimento que adquiri com você e pela sua influência intensa e positiva na minha vida profissional.

Ao meu namorado Kleber, pelo incentivo, carinho, compreensão e atenção, e por sempre ter uma palavra de conforto e estímulo nos momentos que mais precisava. Obrigada também por me ajudar nas programações e nas correções.

Aos meus professores de graduação, especialmente à Prof. Sandra Godoy pela orientação de iniciação científica durante minha graduação e pela amizade.

Aos meus amigos de longe e de perto, novos ou antigos, que de alguma forma contribuíram com este trabalho.

Aos professores de estatística do ICMC, Mariana Cúri, Reiko Aoki, Mário de Castro, Marinho Andrade, Vicente Garibay, pela atenção e ajuda durante o mestrado. Aos funcionários do ICMC pela dedicação.

À Fapesp e ao CNPq pelo apoio financeiro durante o desenvolvimento deste trabalho. 


\section{Resumo}

Em diversas análises estatísticas, nos deparamos com dados multinomiais, dos quais precisamos analisar o comportamento ao longo do tempo e sua relação com fatores determinantes. Os métodos clássicos para modelos de regressão multinomiais consistem em utilizar a estrutura de modelos lineares generalizados para desenvolver tais modelos McCullagh \& Nelder (1989). No entanto, este enfoque apresenta algumas desvantagens como não admiter a incidência de zeros em nenhuma categoria, a hipótese da proporcionalidade da razão de chances e o fato de não serem modelos adequados para análise de dados censurados. Com o objetivo de analisar dados multinomiais com essas características propomos um modelo que é uma extensão do modelo de intensidade multiplicativo desenvolvido por Aalen (1978) e apresentado em Fleming \& Harrington (2005), para variáveis aleatórias multinomiais. Com isso, ao invés de modelarmos as probabilidades associadas às categorias, como nos métodos clássicos, modelamos a função intensidade associada à variável aleatória multinomial. Através do critério martingale, estimamos os parâmetros do modelo ajustado e propomos testes de hipóteses para estes parâmetros para uma e duas populações. O teste para comparação de duas populações é baseado na estatística de logrank. 


\section{Abstract}

In several applications, we want to analyze the behavior of multinomial datas over the time and its relationship with important factors. The classic methods commonly used for multinomial regression models are based in the generalized linear model framework. However, this models presents some disadvantages such that: it does not admit the incidence of zeros in any category, the assumption of proportionality of odds ratio and the fact that they are not appropriate models to analyze censored data. For multinomial data analyses with this characteristics, we propose a model that it is an extension of the multiplicative intensity model developed by Aalen to random multinomial variables. Therefore, instead of modeling the categorical probabilities, as in the classics methods, we modeled the intensity fuction associated with the multinomial variable. Using the martingale criterion, we estimate the models parameters and propose hypothesis testing for these parameters for one and two populations. The test for comparing two populations is based in the logrank statistics. 


\section{Sumário}

1 Introdução 1

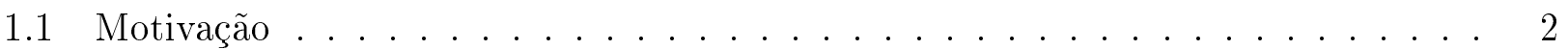

1.2 Estrutura da Dissertação . . . . . . . . . . . . . . . . . . 4

2 Modelo Multinomial $\quad 5$

2.1 Introdução . . . . . . . . . . . . . . . . . . 5

2.2 Espaço de Cantor . . . . . . . . . . . . . . . . . . . . 5

2.3 Variável Aleatória . . . . . . . . . . . . . . . . . 10

2.4 Produto Finito do Espaço Mensurável $\left(S^{\infty}, \mathcal{A}\right) \ldots \ldots$. . . . . . . . . 12

2.5 Probabilidade Condicional . . . . . . . . . . . . . . . . 13

2.6 Cálculo Estocástico . . . . . . . . . . . . . . . . 15

2.6.1 Martingale Array Difference ................. 19

2.7 Integral Estocástica . . . . . . . . . . . . . . . . . 19

2.8 Decomposição de Doob-Meyer . . . . . . . . . . . . . . . . 21

2.8.1 Processo Variação Quadrática . . . . . . . . . . . . . 23

3 Modelo de Intensidade Multiplicativo $\quad 27$

3.1 Introdução . . . . . . . . . . . . . . . . . 27

3.2 Desenvolvimento do Modelo . . . . . . . . . . . . . . 27

3.3 Inferência no Modelo de Intensidade Multiplicativo . . . . . . . . . . . . 33

3.3.1 Propriedades dos estimadores para amostras finitas . . . . . . . . 37

3.3.2 Propriedades assintóticas . . . . . . . . . . . . . 42 
4 Distribuição Assintótica de Integrais Estocásticas 45

4.1 Amostra Única . . . . . . . . . . . . . . . . . . . 45

4.2 Duas Amostras . . . . . . . . . . . . . . . . . 52

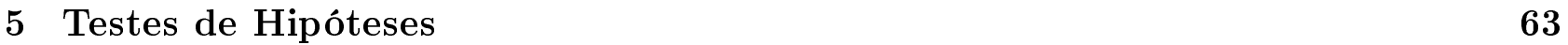

5.1 Introdução . . . . . . . . . . . . . . . . . . 63

5.2 Teste de Hipóteses para uma População . . . . . . . . . . . . . . . . . 63

5.3 Teste de Hipóteses para Duas amostras . . . . . . . . . . . . . . 65

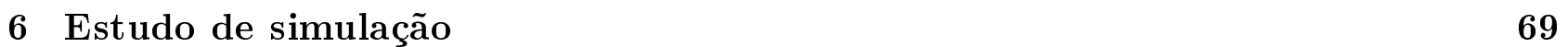

6.1 Introdução . . . . . . . . . . . . . . . . . . 69

6.2 Modelo na ausência de censura . . . . . . . . . . . . . . . . 69

6.2.1 Avaliação da qualidade dos estimadores . . . . . . . . . . . . . 71

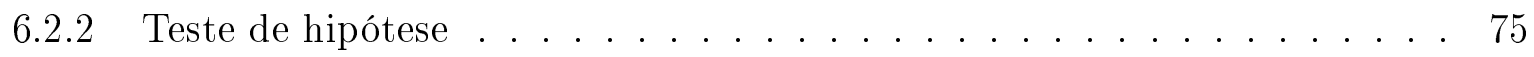

6.3 Análise na presença de censura ..................... 78

6.3.1 Avaliação da qualidade dos estimadores . . . . . . . . . . . . . . 80

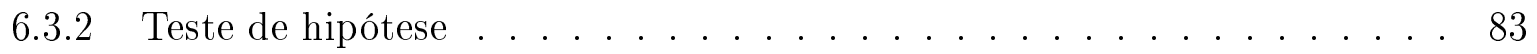

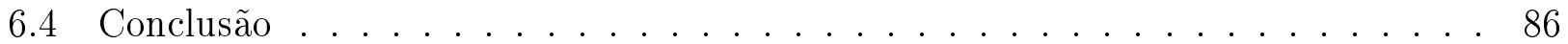

7 Aplicação $\quad 87$

7.1 Introdução . . . . . . . . . . . . . . . . . . 87

7.2 Análise Descritiva da Evasão dos Alunos de Graduação da USP . . . . . . . . 87

7.3 Aplicação do Modelo de Intensidade Multiplicativo . . . . . . . . . . . . 95

7.3.1 Comparação entre anos . . . . . . . . . . . . . 96

7.3 .2 Comparação entre sexos . . . . . . . . . . . . 104

7.3.3 Comparação entre períodos .................... 106

7.3.4 Comparação entre áreas . . . . . . . . . . . . . 108

7.3.5 Portal Evasão . . . . . . . . . . . . . . . . . 112

7.4 Conclusão . . . . . . . . . . . . . . . . . . . 114

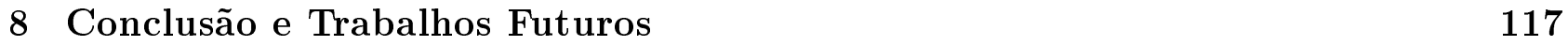


$\begin{array}{lr}\text { A Espaço de Cantor } & 119\end{array}$

Referências Bibliográficas 


\section{Capítulo 1}

\section{Introdução}

Em diversas situações práticas temos o interesse de analisar o comportamento de dados multinomiais (polytomous ou polytochomous) ao longo do tempo e sua relação com fatores determinantes. Uma variável aleatória é multinomial, conforme McCullagh \& Nelder (1989), se esta assume um número finito de valores, aos quais nos referimos como categorias ou classes.

Modelos de regressão multinomial são utilizados para identificar possíveis relações entre a variável resposta multinomial e os fatores associados. Métodos clássicos para modelos de regressão multinomial, como podemos encontrar em McCullagh \& Nelder (1989) e Hosmer \& Lemeshow (1989), consistem em utilizar a estrutura de modelos lineares generalizados para desenvolver modelos de regressão multinomiais. Dependendo da classificação dada à variável multinomial (intervalar, nominal ou ordinal) são propostas diferentes funções de ligação e estratégias para a locação de efeitos.

No entanto, este enfoque apresenta algumas desvantagens, sendo uma delas o fato de que o modelo logístico multinomial não admite a incidência de zeros em nenhuma categoria. Uma possível solução é a forma diferenciada de se lidar com o modelo multinomial proposta por Begg \& Gray (1984) e estendida por Rom \& Cohen (1995) que consiste em ajustar diversos modelos binários, ou seja, os parâmetros de uma regressão logísitca multinomial são estimados utilizando regressões binárias.

Outra hipótese importante assumida na modelagem clássica é a proporcionalidade da razão de chances (odds ratio). Contudo, em muitos conjuntos de dados esta hipótese é inadequada, o que nos leva a desenvolver métodos apropriados para tratar o caso em que a razão de chances 
não são proporcionais. Outra desvantagen dos métodos clássicos de regressão multinomial importante neste estudo é o fato de que esses modelos não são adequados para análise de dados censurados.

Com o objetivo de analisar dados multinomiais com as características descritas acima, categorias nulas, razão de chances não proporcionais e dados censurados, propomos um modelo que é uma extensão do modelo de intensidade multiplicativo desenvolvido por Aalen (1978) e apresentado em Fleming \& Harrington (2005), para variáveis aleatórias multinomiais. O modelo sugerido é também adequado para análise de dados multinomiais que atendem às hipóteses necessárias no modelo de regressão multinomial clássico.

Estimamos os parâmetros do modelo utilizando o critério de martingale e desenvolvemos um estudo das propriedades assintóticas e de amostras finitas. Os estimadores encontrados correspondem aos estimadores empíricos da função de distribuição acumulada, que coincide com o estimador de Kaplan-Meier. Propomos testes de hipóteses para comparação dos parâmetros do modelo por meio da teoria assintótica desenvolvida. Realizamos também estudos de simulação para avaliar as propriedades dos estimadores e a qualidade dos testes de hipóteses. Finalmente, aplicamos o modelo proposto para analisar o comportamento da evasão dos alunos de graduação da Universidade de São Paulo.

\subsection{Motivação}

Utilizamos o modelo proposto neste trabalho para analisar o conjunto de dados reais referente a alunos de graduação da Universidade de São Paulo. O interesse é estudar o tempo até a evasão dos alunos de graduação. Para isso, consideremos o conjunto de dados da graduação da USP (Fonte: JúpiterWeb) referente aos alunos ingressantes por vestibular entre 1999 e 2008.

Neste conjunto de dados temos informações sobre o aluno ingressante e sobre o curso no qual ele está matriculado. A variável de interesse é a que determina a última ocorrência do aluno até o fechamento do segundo semestre de 2008, que é a data do fim do estudo. A última ocorrência pode ser dividida em 5 classes: conclusão, evasão, em andamento, falecimento, migração. Na Tabela 1.1 temos a quantidade de alunos em cada uma dessas classes por ano e o número de ingressantes em cada ano. 
Tabela 1.1: Quantidade de alunos em cada uma das classes de última ocorrência (em andamento, conclusão, evasão, falecimento e migração) por ano e número de ingressantes em cada ano.

\begin{tabular}{c|rrrrrr|c}
\hline & \multicolumn{5}{|c|}{ Última ocorrência } & Número de \\
Ano de ingresso & Em andamento & Conclusão & Evasão & Falecimento & Migração & ingressantes \\
\hline 1999 & 126 & 5096 & 1560 & 6 & 282 & 7070 \\
2000 & 255 & 5148 & 1481 & 12 & 311 & 7207 \\
2001 & 422 & 5445 & 1248 & 7 & 336 & 7458 \\
2002 & 921 & 5465 & 1278 & 11 & 318 & 7993 \\
2003 & 1900 & 5055 & 1179 & 9 & 381 & 8524 \\
2004 & 3932 & 3343 & 1102 & 5 & 388 & 8770 \\
2005 & 6970 & 1205 & 1180 & 6 & 416 & 9777 \\
2006 & 8848 & 13 & 877 & 6 & 425 & 10169 \\
2007 & 9169 & 2 & 671 & 4 & 343 & 10189 \\
2008 & 9657 & 1 & 485 & 1 & 169 & 10313 \\
\hline
\end{tabular}

O termo evasão foi definido pela Pró-Reitoria de graduação da USP e se refere ao aluno que se desligou da USP a seu pedido ou por motivos administrativos (3 semestres sem matrícula, jubilamento, transferência externa, outros). Migração se refere ao aluno que efetuou transferência interna e o termo em adamento se refere ao aluno ativo no fim do estudo.

O objetivo deste trabalho é analisar o número de semestres até a ocorrência de evasão. Para isso, definimos que o início da contagem do número de semestres até a evasão será sempre o ano em que o aluno ingressou na USP e, a data limite, quando o estudo se encerra, é o $2^{\circ}$ semestre de 2008 .

O evento de interesse é a evasão. Os eventos migração, transferência, falecimento e conclusão, correspondem aos eventos de censura. A cada aluno associamos um variável aleatória multinomial que determina o número de semestres até a última ocorrência. Iremos então avaliar o comportamento desta variável aleatória multinomial ao longo do tempo de forma a estudar o tempo até a evasão e sua relação com atributos associados ao aluno, como sexo, período e área. 


\subsection{Estrutura da Dissertação}

A presente dissertação de mestrado está dividida em oito capítulos. No segundo capítulo, contruímos um modelo probabilístico para acomodar uma variável aleatória multinomial e apresentamos definições e resultados de probabilidade, esperança condicional, cálculo estocástico, integral estocástica e decomposição de Doob-Meyer, que serão utilizados nos capítulos posteriores.

No terceiro capítulo, apresentamos o modelo de intensidade multiplicativo proposto neste trabalho, obtemos os estimadores dos parâmetros desse modelo e estudamos suas propriedades de amostras finitas e assintóticas. O quarto capítulo contém um estudo da distribuição assintótica de integrais estocásticas. Este estudo foi de fundamental importância para o desenvolvimento de testes de hipóteses para comparações dos parâmetros do modelo, obtidos no quinto capítulo.

No sexto capítulo, avaliamos numericamente a qualidade das aproximações utilizadas nas inferências sobre os parâmetros do modelo de intensidade multiplicativo por meio um estudo de simulação. O interesse nesse capítulo é comprovar, através do estudo de simulação, as proriedades dos estimadores provadas no capítulo anterior, além de avaliar a qualidade dos testes de hipóteses.

No sétimo capítulo, analisamos o conjunto de dados de alunos de graduação da Universidade de São Paulo utilizando o modelo de intensidade multiplicativo. Finalmente, o oitavo capítulo apresenta conclusões dos resultados obtidos nesse trabalho e propostas de trabalhos futuros. 


\section{Capítulo 2}

\section{Modelo Multinomial}

\subsection{Introdução}

Se a resposta associada a um indivíduo ou ítem assume um número finito de valores, temos que a resposta é multinomial (McCullagh \& Nelder (1989)). Os valores que a resposta assume são denominados categorias ou intervalos de classe. As técnicas utilizadas para analisar dados multinomiais dependem do tipo de dado analisado. Os principais tipos de dados multinomiais são: nominal, ordinal e intervalar. O modelo aqui desenvolvido pode ser aplicado para qualquer um destes tipos.

A seguir será contruido um modelo probabilístico para acomodar uma variável aleatória multinomial.

\subsection{Espaço de Cantor}

Consideremos $\eta$ uma função de distribuição de probabilidade tomando valores em $\{1,2, \ldots, k\}$, para $k \in \mathbb{N}$, tal que

- $\eta(i) \geq 0, \forall i=1, \ldots, k$;

- $\eta(1)+\ldots+\eta(k)=1$. 
Na seqüência, construímos uma variável aleatória multinomial a partir da distribuição $\eta$. Seja $S=\{0,1\}$ o espaço formado por dois elementos, 0 e 1 , e

$$
S^{\infty}=S \times S \times \ldots
$$

O espaço $S^{\infty}$ é conhecido como espaço de Cantor. Uma descrição detalhada deste espaço pode ser encontrada em Leao et al. (2005). Seja $\pi_{k}: S^{\infty} \rightarrow S$ a projeção na $k$-ésima coordenada $(k \in \mathbb{N})$. A partir da projeção coordenada, defimos a classe de cilindros de $S^{\infty}$ com base em $S$

$$
\mathcal{H}=\left\{\pi_{k}^{-1}\left(\left\{\omega_{k}\right\}\right): \omega_{k} \in S \text { e } k \in \mathbb{N}\right\}
$$

Da definição de $\mathcal{H}$ obtemos os seguintes resultados:

1. $\pi_{k}^{-1}(\{0\}) \cap \pi_{k}^{-1}(\{1\})=\emptyset, \forall k \in \mathbb{N}$.

2. $\pi_{k}^{-1}(\{0\}) \cup \pi_{k}^{-1}(\{1\})=S^{\infty}, \forall k \in \mathbb{N}$.

Na classe de cilindros de dimensão $1, \mathcal{H}$, definimos a função de probabilidade $P: \mathcal{H} \rightarrow[0,1]$ como

$$
P\left[\pi_{k}^{-1}\left(\left\{\omega_{k}\right\}\right)\right]=\frac{1}{2}
$$

A partir de $\mathcal{H}$, construímos a classe $\Delta$

$$
\begin{aligned}
\Delta & =\left\{\pi_{v_{1}}^{-1}\left(\left\{\omega_{v_{1}}\right\}\right) \cap \ldots \cap \pi_{v_{n}}^{-1}\left(\left\{\omega_{v_{n}}\right\}\right):\left(\omega_{v_{1}}, \ldots, \omega_{v_{n}}\right) \in S^{n}, v_{i} \in \mathbb{N}, i=1, \ldots, n \text { e } n \in \mathbb{N}\right\} \cup\left\{\emptyset, S^{\infty}\right\} \\
& =\left\{D \subset S^{\infty}: D=B_{1} \cap B_{2} \cap \ldots \cap B_{n}, \quad B_{i} \in \mathcal{H}, \quad i=1, \ldots, n, \quad n \in \mathbb{N} ; \emptyset, S^{\infty}\right\} .
\end{aligned}
$$

Lema 2.1. A classe $\Delta$ é uma semi-álgebra, ou seja,

(i) $\emptyset$ e $S^{\infty} \in \Delta$;

(ii) $\Delta$ é fechada pela interseção finita;

(iii) $S e C \in \Delta$, então $C^{C} \notin \Delta$. Porém,

$$
C^{c}=D_{1} \cup D_{2} \cup \ldots \cup D_{n}, \quad D_{i} \cap D_{l}=\emptyset, \quad i \neq l \text { e } D_{i} \in \Delta .
$$

Demonstração. (i) Definição. 
(ii) Sejam $\Delta_{1}, \Delta_{2}, \ldots, \Delta_{n} \in \Delta$ tal que

$$
\begin{aligned}
\Delta_{1} & =B_{1}^{1} \cap B_{2}^{1} \cap \ldots \cap B_{k_{1}}^{1} \\
\Delta_{2} & =B_{1}^{2} \cap B_{2}^{2} \cap \ldots \cap B_{k_{2}}^{2} \\
& \vdots \\
\Delta_{n} & =B_{1}^{n} \cap B_{2}^{n} \cap \ldots \cap B_{k_{n}}^{n}, \quad B_{l}^{i} \in \mathcal{H}, \quad l=1, \ldots, k_{i}, \quad i=1, \ldots, n, \quad k_{i}, n \in \mathbb{N} .
\end{aligned}
$$

Assim,

$$
\bigcap_{i=1}^{n} \Delta_{i}=\bigcap_{i=1}^{n} \bigcap_{l=1}^{k_{i}} B_{l}^{i}
$$

Portanto, $\bigcap_{i=1}^{n} \Delta_{i}$ pode ser escrita como interseção finita de elementos de $\mathcal{H}$. Logo, $\bigcap_{i=1}^{n} \Delta_{i} \in \Delta$.

(iii) Consideremos $C \in \Delta$. Então,

$$
C=B_{1} \cap B_{2} \cap \ldots \cap B_{n}, \quad B_{i} \in \mathcal{H}, \quad i=1, \ldots, n, \quad n \in \mathbb{N} .
$$

Assim,

$$
C^{c}=S^{\infty}-\left\{B_{1} \cap B_{2} \cap \ldots \cap B_{n}\right\} .
$$

Como $B_{i} \in \mathcal{H}$, para determinado $\omega_{k_{i}} \in S, \quad k_{i} \in \mathbb{N}, \quad i=1, \ldots, n$ e $n \in \mathbb{N}$, obtemos

$$
B_{i}=\pi_{k_{i}}^{-1}\left(\left\{\omega_{k_{i}}\right\}\right) .
$$

Portanto,

$$
\begin{aligned}
C^{c} & =S^{\infty}-\left\{\pi_{k_{1}}^{-1}\left(\left\{\omega_{k_{1}}\right\}\right) \cap \ldots \pi_{k_{n}}^{-1}\left(\left\{\omega_{k_{n}}\right\}\right)\right\} \\
& =\left\{\mathbf{x}=\left(x_{n}\right)_{n} \in S^{\infty}: \exists j, \quad j \in\{1, \ldots, n\}, \text { tal que } x_{k_{j}} \neq \omega_{k_{j}}\right\}
\end{aligned}
$$

Consideremos agora o conjunto

$$
\Gamma=S^{n}-\left\{\left(\omega_{k_{1}}, \omega_{k_{2}}, \ldots, \omega_{k_{n}}\right)\right\} .
$$

Daí temos que

$$
C^{c}=\bigcup_{\mathfrak{H} \in \Gamma}\left\{\mathbf{x}=\left(x_{n}\right)_{n} \in S^{\infty}: \pi_{k_{i}}(\mathbf{x})=\mathfrak{H}_{i}, \quad i=1, \ldots, n, \quad n \in \mathbb{N}\right\} .
$$

Portanto, $C^{c}$ é união finita e disjunta de elementos de $\Delta$. 
Podemos estender a probabilidade $P$ para a semi-álgebra de cilindros $\Delta, P: \Delta \rightarrow[0,1]$ de tal forma que $P(\emptyset)=0, P\left(S^{\infty}\right)=1$ e

$$
P(B)=\prod_{i=1}^{j} P\left[\pi_{k_{i}}^{-1}\left(\left\{\omega_{k_{i}}\right\}\right)\right]=\left(\frac{1}{2}\right)^{j},
$$

para todo $\left(\omega_{v_{1}}, \ldots, \omega_{v_{j}}\right) \in S^{j}$ e $C \in \Delta$.

Para obtermos uma álgebra gerada pelos cilindros, basta aplicar as operações de conjunto definidas no item (iii) do Lema 2.1 aos elementos de $\Delta$. Assim, denotamos por $\mathcal{A}$ a classe formada pela união finita disjunta de elementos de $\Delta$,

$$
\mathcal{A}=\left\{A \in S^{\infty}: A=\bigcup_{i=1}^{n} C_{i}, \quad C_{i} \in \Delta, \quad C_{k} \cap C_{j}=\emptyset, \quad k, j=1, \ldots, n, \quad k \neq j\right\} .
$$

Lema 2.2. A classe $\mathcal{A}$ é a álgebra gerada por $\Delta$, ou seja,

(i) $S^{\infty}, \emptyset \in \mathcal{A}$.

(ii) A classe $\mathcal{A}$ é fechada pela complementação.

(iii) A classe $\mathcal{A}$ é fechada pela interseção finita.

(iv) Toda álgebra $\mathcal{B}$ de conjuntos de $S^{\infty}$ que contém $\Delta$ também contém $\mathcal{A}$.

Demonstração. (i) $S^{\infty}, \emptyset \in \mathcal{A}$, pois $\Delta \in \mathcal{A}$.

(ii) Mostremos que se $A \in \mathcal{A}$ então $A^{c} \in \mathcal{A}$.

Considere $A \in \mathcal{A}$. Assim,

$$
A=\bigcup_{i=1}^{n} C_{i}, \quad C_{i} \in \Delta, \quad C_{k} \cap C_{j}=\emptyset, \quad k, j=1, \ldots, n, \quad k \neq j .
$$

Conseqüentemente,

$$
A^{c}=\bigcap_{i=1}^{n} C_{i}^{c}
$$

Pelo item (iii) do Lema 2.1 temos que

$$
C_{i}^{c}=\bigcup_{j_{i}=1}^{m_{i}} D_{j_{i}}^{i}, D_{j_{i}}^{i} \in \Delta, D_{l}^{i} \cap D_{s}^{i}=\emptyset, l \neq s, j_{i}=1, \ldots, m_{i}, m_{i} \in \mathbb{N}, i=1, \ldots, n .
$$


Portanto,

$$
\begin{aligned}
A^{c} & =\bigcap_{i=1}^{n}\left(\bigcup_{j_{i}=1}^{m_{i}} D_{j}^{i}\right)=\left\{\bigcup_{j_{1}=1}^{m_{1}} D_{j_{1}}^{1}\right\} \cap \ldots \cap\left\{\bigcup_{j_{n}=1}^{m_{n}} D_{j_{n}}^{n}\right\} \\
& =\bigcup_{\left\{j_{1}, \ldots, j_{n}\right\} \in M}\left(D_{j_{1}}^{1} \cap \ldots \cap D_{j_{n}}^{n}\right)
\end{aligned}
$$

sendo $M=\left\{1, \ldots, m_{1}\right\} \times \ldots \times\left\{1, \ldots, m_{n}\right\}$.

Observe que os conjuntos

$$
D_{j_{1}}^{1} \cap \ldots \cap D_{j_{n}}^{n}
$$

com $\left(j_{1}, j_{2}, \ldots, j_{n}\right) \in M$, são disjuntos. Além disso, como $\Delta$ é fechada pela interseção, obtemos que

$$
D_{j_{1}}^{1} \cap \ldots \cap D_{j_{n}}^{n} \in \Delta
$$

$\operatorname{para}\left(j_{1}, \ldots, j_{n}\right) \in M$

Assim, temos que $A^{c}$ é união finta disjunta de elementos de $\Delta$, ou seja, $A^{c} \in \mathcal{A}$.

(iii) Sejam $A, B \in \mathcal{A}$. Então,

$$
A=\bigcup_{i=1}^{n_{A}} C_{i}^{A}, \quad B=\bigcup_{j=1}^{n_{B}} C_{i}^{C}
$$

$\operatorname{com} C_{i}^{A} \in \Delta, \quad C_{j}^{B} \in \Delta, \quad C_{s}^{L} \cap C_{l}^{L}=\emptyset$ para $s \neq l, \quad s, l \in\left\{1, \ldots, n_{L}\right\}, \quad L=A, B$.

Assim temos que

$$
A \cap B=\left[\bigcup_{i=1}^{n_{A}} C_{i}^{A}\right] \cap\left[\bigcup_{j=1}^{n_{B}} C_{j}^{B}\right]=\bigcup_{i=1}^{n_{A}} \bigcup_{j=1}^{n_{B}}\left[C_{i}^{A} \cap C_{j}^{B}\right] .
$$

Note que $\left[C_{i_{1}}^{A} \cap C_{j_{1}}^{B}\right] \cap\left[C_{i_{2}}^{A} \cap C_{j_{2}}^{B}\right]=\emptyset$, se $i_{1} \neq i_{2}$ ou $j_{1} \neq j_{2}$. Além disso, $\Delta$ é fechada pela interseção finita, portanto, $A \cap B$ é união finita disjunta de elementos de $\Delta$. Logo, $A \cap B \in \mathcal{A}$. Portanto, $\mathcal{A}$ é fechada pela interseção finita.

Na seqüência construímos uma distribuição de probabilidade sobre a álgebra gerada pelos cilindros. Consideremos a probabilidade definida em 2.1, $P: \Delta \rightarrow[0,1]$ tal que

$$
P\left[\pi_{v_{1}}^{-1}\left(\left\{\omega_{v_{1}}\right\}\right) \cap \ldots \cap \pi_{v_{j}}^{-1}\left(\left\{\omega_{v_{j}}\right\}\right)\right]=\left(\frac{1}{2}\right)^{j},
$$

Assim, tomando $C \in \mathcal{A}$, sabemos que existe uma coleção finita $\left\{D_{i}\right\}_{i=1}^{n} \subset \Delta$ tal que 
(i) $D_{i} \cap D_{l}=\emptyset, i \neq l$

(ii) $\bigcup_{i=1}^{n} D_{i}=C$.

Desta forma, a função de conjunto $P$ é estendida sobre a álgebra de cilindros $\mathcal{A}$ por

$$
P(C)=\sum_{i=1}^{n} P\left(D_{i}\right)
$$

Observemos que a função $P$ está bem definida. De fato, consideremos $\sum_{i=1}^{n} D_{i}=\sum_{j=1}^{m} B_{j}$ para $m, n \in \mathbb{N}$ e $\left\{D_{i}\right\}_{i=1}^{n},\left\{B_{i}\right\}_{j=1}^{m} \in \Delta$. Então,

$$
\sum_{i=1}^{n} P\left(D_{i}\right)=\sum_{i=1}^{n} \sum_{j=1}^{m} P\left[D_{i} \cap B_{j}\right]=\sum_{j=1}^{m} \sum_{i=1}^{n} P\left[D_{i} \cap B_{j}\right]=\sum_{j=1}^{m} B_{j} .
$$

Finalmente, via o Teorema de extensão de Caratheodory, obtemos uma única probabilidade sobre a $\sigma$-álgebra $\mathcal{F}$ gerada pela álgebra $\mathcal{A}$. Como as variáveis aleatórias que serão estudadas nesta dissertação são multinomiais, todas as análises realizadas na seqüência serão desenvolvidas sobre a álgebra $\mathcal{A}$. Com isso, denotamos por $\left(S^{\infty}, \mathcal{A}, P\right)$ nosso espaço de probabilidade.

\subsection{Variável Aleatória}

Nesta seção construímos uma variável aleatória associada à distribuição de probabilidade $\eta$. Primeiramente, definimos que uma partição $\mathcal{K}$ do $S^{\infty}$ é uma classe finita de conjuntos $K_{1}, \ldots, K_{n}$ satisfazendo

(i) $K_{i} \in \mathcal{A}, \quad i=1, \ldots, n$;

(ii) $\cup_{i=1}^{n} K_{i}=S^{\infty}$;

(iii) $K_{j} \cap K_{l}=\emptyset, \quad j, l=1, \ldots, n$ e $j \neq l$.

Denotamos por $S(\mathcal{A})$ conjunto de todas as funções $X: S^{\infty} \rightarrow \mathbb{R}$ tais que

$$
X=\sum_{i=1}^{n} c_{i} \mathbb{1}_{\left\{K_{i}\right\}}
$$

com $K_{1}, \ldots, K_{n}$ uma partição do $S^{\infty}$ e $c_{i} \in \mathbb{R}, \quad i=1, \ldots, n$. Os elementos de $S(\mathcal{A})$ são denominados variáveis aleatórias (ou funções mensuráveis). 
Teorema 2.1. Consideremos o espaço de probabilidade $\left(S^{\infty}, \mathcal{A}, P\right)$.

(i) Dada uma variável aleatória $X$ assumindo valores no espaço finito $\left\{c_{1}, \ldots, c_{n}: c_{i} \in\right.$ $\mathbb{R}$ e $n \in \mathbb{N}\}$ existe uma função $\eta$ associada à $X$ tal que

$$
P\left[\omega: X(\omega)=c_{i}\right]=\eta(i)=\frac{j_{i}}{2^{j}},
$$

para $j_{i} \in\left\{0,1, \ldots, 2^{j}\right\}$ e $j \in \mathbb{N}$.

(ii) Dada uma distribuição de probabilidade discreta $\eta$ sobre o espaço finito $\left\{c_{1}, \ldots, c_{n}: c_{i} \in\right.$ $\mathbb{R}$ e $n \in \mathbb{N}\}$ satisfazendo $\eta(i)=\frac{j_{i}}{2^{j}}$ para $j_{i} \in\left\{0,1, \ldots, 2^{j}\right\}$ e $j \in \mathbb{N}$, existe uma variável aleatória $X: S^{\infty} \rightarrow \mathbb{R}$ satisfazedo

$$
P\left[\omega: X(\omega)=c_{i}\right]=\eta(i)
$$

Demonstração. Consideremos $\left\{B_{1}, B_{2}, \ldots, B_{2^{j}}\right\}$ uma enumeração qualquer tal que

$$
\Delta_{j}=\left\{\pi_{1}^{-1}\left(\left\{\omega_{1}\right\}\right) \cap \ldots \cap \pi_{1}^{-1}\left(\left\{\omega_{j}\right\}\right):\left(\omega_{1}, \omega_{2}, \ldots, \omega_{J}\right) \in S^{j}\right\}=\left\{B_{1}, B_{2}, \ldots, B_{2^{j}}\right\} .
$$

Tome,

$$
\begin{aligned}
A_{1} & =B_{1} \cup \cdots \cup B_{j_{1}} \\
& \vdots \\
A_{i} & =B_{j_{1}+\cdots+j_{i-1}} \cup \cdots \cup B_{j_{1}+\cdots+j_{i}},
\end{aligned}
$$

para $i=1,2, \ldots, n$. Assim, obtemos uma variável aleatória $X$

$$
X(\omega)=\sum_{i=1}^{n} i \mathbb{1}_{\left\{A_{i}\right\}}(\omega)
$$

tal que

$$
\eta(i)=P\left[w \in S^{\infty}: X(w)=i\right]=P\left(A_{i}\right)=\frac{j_{i}}{2^{j}} .
$$

A probabilidade imagem $P$ definida em (2.2) é a distribuição de probabilidade discreta $\eta(i)$ associada à variável aleatória $X$ definida anteriormente.

Por outro lado, dado $X=\sum_{i=1}^{n} c_{i} \mathbb{1}_{\left\{K_{i}\right\}}$ uma variável aleatória obtemos que $\eta(i)=P\left[K_{i}\right]$.

Observe que a representação da variável aleatória $X$ não é única: qualquer permutação dos elementos de $\Delta_{j}$ nos conduz a uma variável aleatória distinta. Por isso, fixamos a enumeração definida por $\Delta_{j}$. 


\subsection{Produto Finito do Espaço Mensurável $\left(S^{\infty}, \mathcal{A}\right)$}

A classe de variáveis aleatória que vamos estudar evolui ao longo do tempo através de uma estrutura que envolve espaços mensuráveis produto. Assim, vamos contruir esses espaços e estudar algumas de suas propriedades. Denotamos por

$$
\left(S^{\infty}\right)^{n}=\underbrace{S^{\infty} \times \ldots \times S^{\infty}}_{n \text { vezes }}
$$

o produto cartesiano do $S^{\infty}$ com ele mesmo $n$ vezes.

Considere $\pi_{i}:\left(S^{\infty}\right)^{n} \rightarrow S^{\infty}$ a projeção na $i$-ésima coordenada, para $i=1, \ldots, n$. Seja $A$ um conjunto arbitrário de $\left(S^{\infty}\right)^{n}, L \subset\{1,2, \cdots, n\}$ e $\omega_{u} \in S^{\infty}$, para todo $u \in L$. Definimos a seção de $A \operatorname{em}\left\{\omega_{u}: u \in L\right\}$, por

$$
A_{\left\{\omega_{u} ; u \in L\right\}}=\left\{\left\{\omega_{i} ; i \in L^{c}\right\}:\left(\omega_{1}, \cdots, \omega_{n}\right) \in A\right\}
$$

Para todo $\left\{\omega_{u}: u \in L\right\}$ fixos, a transformação $A \rightarrow A_{\left\{\omega_{u}: u \in L\right\}}$ preserva as operações de união e interseção finitas, e complementação. Na realidade, se tomarmos $\left\{A_{k}\right\}$ uma família finita qualquer de subconjuntos de $\left(S^{\infty}\right)^{n}$, temos que

$$
\left(\bigcap_{i=1}^{k} A_{i}\right)_{\left\{\omega_{u}: u \in L\right\}}=\bigcap_{i=1}^{k}\left(A_{i}\right)_{\left\{\omega_{u}: u \in L\right\}}
$$

com o mesmo sendo válido para as operações de união finita e complementaçãao. Por exemplo, se tomarmos $n=2$, isto é, $\left(S^{\infty}\right)^{2}$ e $A \subset\left(S^{\infty}\right)^{2}$, obtemos que

$$
A_{\omega_{2}}=\left\{\omega_{1} \in S^{\infty}:\left(\omega_{1}, \omega_{2}\right) \in A\right\}
$$

com $\omega_{2} \in S^{\infty}$. Consideremos o conjunto $L \subset\{1,2, \cdots, n\}$ e $\alpha(L)$ a cardinalidade deste conjunto. Outra operação que utilizaremos é a projeção de subconjuntos $A \subset\left(S^{\infty}\right)^{n}$ sobre $\left(S^{\infty}\right)^{\alpha(L)}$, que definiremos por

$$
\operatorname{Proj}_{\left(S^{\infty}\right)^{\alpha(L)}}[A]=\left\{\left\{\omega_{u}: u \in L\right\}: A_{\left\{\omega_{u} ; u \in L\right\}} \neq \emptyset\right\}
$$

A construção de uma álgebra sobre o espaço $\left(S^{\infty}\right)^{n}$ segue as mesmas linhas de contrução da topologia produto, trocando-se continuidade por mensurabilidade. Para isto, dizemos que um retângulo em $\left(S^{\infty}\right)^{n}$ é um subconjunto, na forma

$$
R=R_{1} \times R_{2} \times \cdots \times R_{n}=\prod_{i=1}^{n} R_{i}=\left\{\left(\omega_{1}, \cdots, \omega_{n}\right): \omega_{i} \in R_{i}\right\}
$$


com $R_{i} \subset S^{\infty}, i=1, \ldots, n$. Observamos que $R$ é vazio se, e somente se, $R_{i}=\emptyset$ para algum $i=0,1, \cdots, n$. Ao aplicarmos operação de seção em um retângulo $R$ de $\left(S^{\infty}\right)^{n}$, obtemos que

$$
R_{\left\{\omega_{u}: u \in L\right\}}=\left\{\begin{array}{ccc}
\prod_{j \in S^{c}} R_{j}, & ; \quad\left\{\omega_{u} ; u \in L\right\} \in \prod_{u \in L} R_{u} \\
\emptyset & ; \quad\left\{\omega_{u} ; u \in L\right\} \notin \prod_{u \in L} R_{u}
\end{array}\right.
$$

Um retângulo $R=R_{1} \times R_{2} \times \cdots \times R_{n}$ em $\left(S^{\infty}\right)^{n}$ é denominado mensurável se $R_{i} \in \mathcal{A}_{i}$ para $1 \leq i \leq n$. Na seqüência, vamos mostrar que a classe de retângulos mensuráveis é uma semi-álgebra.

Lema 2.3. Os retângulos mensuráveis de $\left(S^{\infty}\right)^{n}$ formam uma semi-álgebra.

A partir desta estrutura de semi-álgebra sobre a classe de retângulos mensuráveis podemos construir a álgebra produto, utilizando resultados clássicos da teoria de conjuntos. Se tomarmos uniões finitas disjuntas (2 a 2) de retângulos mensuráveis obtemos uma álgebra $\mathcal{A}^{n}$ de sobconjuntos de $\left(S^{\infty}\right)^{n}$ [ Neveu (1965); prop. I.6.1, pp. 25].

\subsection{Probabilidade Condicional}

Nesta seção definimos conceitos de probabilidade condicional e esperança condicional importantes para o estudo de processos estocásticos em geral. Consideremos $P: \mathcal{A} \rightarrow[0,1]$ a probabilidade como definida na equação 2.1. Sejam $A, B \in \mathcal{A} \operatorname{com} P(B)>0$, então a probabilidade condicional de $A$ dado $B$ é dada por

$$
P[A \mid B]=\frac{P[A \cap B]}{P(B)} .
$$

Seja $\mathcal{K}=\left\{K_{1}, \ldots, K_{n}\right\}$ uma partição. Definimos a probabilidade condicional de $A \in \mathcal{A}$ dado a partição $\mathcal{K}, P[A \mid \mathcal{K}]: S^{\infty} \rightarrow[0,1]$ como

$$
\begin{aligned}
P[A \mid \mathcal{K}](\omega) & =\sum_{i=1}^{n} P\left[A \mid K_{i}\right] \mathbb{1}_{\left\{k_{i}\right\}}(\omega) \\
& =\sum_{i=1}^{n} \frac{P\left[A \cap K_{i}\right]}{P\left(K_{i}\right)} \mathbb{1}_{\left\{K_{i}\right\}}(\omega), \omega \in S^{\infty} .
\end{aligned}
$$


Seja $X \in S(\mathcal{A})$ uma variável aleatória. Definimos a esperança condicional da variável $X$ dado à partição $\mathcal{K}$ como

$$
E[X \mid \mathcal{K}](\omega)=\sum_{i=1}^{n} E\left[X \mid K_{i}\right] \mathbb{1}_{\left\{K_{i}\right\}}(\omega), \omega \in S^{\infty}
$$

no qual

$$
E\left[X \mid K_{i}\right]=\frac{E\left[X \mathbb{1}_{\left\{K_{i}\right\}}\right]}{E\left[\mathbb{1}_{\left\{K_{i}\right\}}\right]}=\frac{E\left[X \mathbb{1}_{\left\{K_{i}\right\}}\right]}{P\left(K_{i}\right)} .
$$

A partir da partição $\mathcal{K}$, obtemos a álgebra $\mathcal{A}(\mathcal{K})$ cujos elementos são formados pela união finita disjunta de elementos de $\mathcal{K}$ mais o conjunto vazio $\emptyset$ e o conjunto $S^{\infty}$. Por outro lado, dado uma sub-álgebra finita $\mathcal{G} \subset \mathcal{A}$, obtemos uma partição $\mathcal{K} \subset \mathcal{A}$ tal que $\mathcal{G}=\mathcal{A}(\mathcal{K})$. Com isso, para toda variável $X \in S(\mathcal{A})$, verificamos que

$$
E[X \mid \mathcal{A}(\mathcal{K})](\omega)=E[X \mid \mathcal{K}](\omega), \omega \in S^{\infty} .
$$

Desta forma, observe que se $X(\omega)=\mathbb{1}_{\{A\}}(\omega)$ para $A$ um conjunto qualquer, então

$$
\begin{aligned}
E[X \mid \mathcal{A}(\mathcal{K})](\omega) & =E\left[\mathbb{1}_{\{A\}} \mid \mathcal{K}\right](\omega)=\sum_{i=1}^{n} E\left[\mathbb{1}_{\{A\}} \mid K_{i}\right] \mathbb{1}_{\left\{K_{i}\right\}}(\omega) \\
& =\sum_{i=1}^{n} \frac{P\left[A \cap K_{i}\right]}{P\left(K_{i}\right)} \mathbb{1}_{\left\{K_{i}\right\}}(\omega) \\
& =P[A \mid \mathcal{K}](\omega)
\end{aligned}
$$

Outras propriedades da esperança condicional:

1. Sejam $X_{1}, X_{2} \in S(\mathcal{A})$ e $a \in \mathbb{R}$, então

$$
E\left[a X_{1}+X_{2} \mid \mathcal{K}\right]=a E\left[X_{1} \mid \mathcal{K}\right]+E\left[X_{2} \mid \mathcal{K}\right] .
$$

2. Se $\mathcal{K}_{1}$ e $\mathcal{K}_{2}$ são partições independetes, então para $X \in S\left(\mathcal{K}_{1}\right)$ temos que

$$
E\left[X \mid \mathcal{K}_{2}\right]=E[X]
$$

3. Seja $X \in S(\mathcal{A}(\mathcal{K}))$. Então

$$
E[X \mid \mathcal{A}(\mathcal{K})](\omega)=X(\omega)
$$




\subsection{Cálculo Estocástico}

Seja $\mathbb{N}_{0}$ o conjunto formado pelos números naturais e pelo elemento zero. Um processo estocástico é uma família de variáveis aleatórias $Z=\left\{Z(n, \cdot): n \in \mathbb{N}_{0}\right\}$ definidas sobre o espaço de probabilidade $\left(S^{\infty}, \mathcal{A}, P\right)$. Uma das principais estratégias para se estudar processos estocásticos, proposta por Doob (1953), consiste em definir uma seqüência de álgebras que correspondem à quantidade de informações que se tem ao longo do tempo $(n)$. Denominamos essa seqüência de álgebras de filtragem.

Definição 2.1. Uma filtragem $\left\{\mathcal{E}_{n}\right\}$ no espaço de probabilidade $\left(S^{\infty}, \mathcal{A}, P\right)$ é definida como uma seqüência de álgebras encaixantes tais que

(i) $\mathcal{E}_{n} \subset \mathcal{A} e$

(ii) $\mathcal{E}_{n} \subset \mathcal{E}_{n+1}, n \in \mathbb{N}$.

Uma das filtragens mais usuais consiste em considerar os cilindros de dimensão finita. Para isto, definimos uma classe de conjuntos $\Delta_{n}$ que contempla os cilindros com base em dimensão menor ou igual a $n$,

$$
\Delta_{n}=\left\{\pi_{1}^{-1}\left(\left\{\omega_{1}\right\}\right) \cap \ldots \cap \pi_{k}^{-1}\left(\left\{\omega_{k}\right\}\right):\left(\omega_{1}, \ldots, \omega_{k}\right) \in S^{k}, k \leq n\right\} \cup\left\{\emptyset, S^{\infty}\right\},
$$

para $n \in \mathbb{N}$. No espaço de probabilidade $\left(S^{\infty}, \mathcal{A}, P\right)$, considere a filtragem $\left\{A_{n}\right\}$

$$
\begin{aligned}
\mathcal{A}_{0} & =\left\{\emptyset, S^{\infty}\right\} \\
\mathcal{A}_{1} & =\left\{\emptyset, \pi_{1}^{-1}(\{0\}), \pi_{1}^{-1}(\{1\}), S^{\infty}\right\} \\
& \vdots \\
\mathcal{A}_{n} & =\text { união finita disjunta de elementos de } \Delta_{n}, \quad n \in \mathbb{N} .
\end{aligned}
$$

Neste caso, $\left\{\mathcal{A}_{n}\right\}$ é a filtragem gerada pela seqüência de partições $\left\{\Delta_{n}\right\}$.

Definição 2.2. Um processo estocástico $Z$ é adaptado para a filtragem $\left\{\mathcal{E}_{n}\right\}$ se

$$
Z(n, .) \in S\left(\mathcal{E}_{n}\right), \forall n \in \mathbb{N} .
$$


Um dos principais processos estudados nesta dissertação é o processo de contagem. Para isso, tomamos $X$ uma variável aleatória assumindo valores $\{1,2, \ldots, k\}$, com representação

$$
X(\omega)=\sum_{i=1}^{k} i \mathbb{1}_{\left\{A_{i}\right\}}
$$

no qual $A_{1}, \ldots, A_{k}$ é uma partição do $S^{\infty}$. O processo de contagem associado à variável aleatória $X$ é definido por

$$
N(i, \omega)=\mathbb{1}_{\{X(\omega) \leq i\}}, i=1, \ldots, k \text { e } \omega \in S^{\infty} .
$$

A seguir, definimos uma seqüência de partições associada ao processo de contagem $N$,

$$
\begin{aligned}
\Delta_{0}^{N} & =\left\{\emptyset, S^{\infty}\right\} \\
\Delta_{1}^{N} & =\{\{N(1, .)=0\} ;\{N(1, .)=1\}\} \\
& =\left\{A_{1} ; A_{1}^{c}\right\} \\
\vdots & \\
\Delta_{n}^{N} & =\{\{N(1, .)=0, \ldots, N(n, .)=0\},\{N(1, .)=1, \ldots, N(n, .)=0\}, \ldots,\{N(1, .)=1, \ldots, N(n, .)=1\}\} \\
& \left.=\left\{\left(A_{1} \cup A_{2} \cup \ldots \cup A_{n}\right)^{c}\right\},\left\{A_{1}^{c} \cup A_{2} \cup \ldots \cup A_{n}\right\}, \ldots,\left\{A_{1} \cup A_{2} \cup \ldots \cup A_{n}\right\}\right\}
\end{aligned}
$$

A filtragem $\left\{\mathcal{A}_{n}^{N}\right\}$, também denominada filtragem interna do processo de contagem $N$, é a filtragem gerada pela seqüência de partições $\left\{\Delta_{n}^{N}\right\}$.

Definição 2.3. Seja $Z$ um processo estocástico e $\left\{\mathcal{E}_{n}\right\}$ uma filtragem definida no espaço de probabilidade $\left(S^{\infty}, \mathcal{A}, P\right)$. Z é um martingale se

(i) $Z(n,$.$) é adaptado para a filtragem \left\{\mathcal{E}_{n}\right\}$;

(ii) $|E[Z(n,)]|<.\infty, \quad \forall n \in \mathbb{N}$;

(iii) $E\left[Z(n+1,) \mid. \mathcal{E}_{n}\right]=Z_{n}$.

$Z$ é um supermartingale se forem válidas as propriedades (i), (ii) e

$$
E\left[Z(n+1, .) \mid \mathcal{E}_{n}\right] \leq Z_{n}
$$

$Z$ é um submartingale se forem válidas (i), (ii) e

$$
E\left[Z(n+1, .) \mid \mathcal{E}_{n}\right] \geq Z_{n}
$$


Se um processo estocástico $Z$ é um supermartingale, então $-Z$ é um submartingale. Com isso, se um processo estocástico é um supermartingale e um submartingale, então ele é um martingale. Além disso, se $Z$ é supermartingale a esperança de $Z(n, \cdot)$ é não crescente, isto é,

$$
\mathrm{E}[Z(n, \cdot)] \geq \mathrm{E}[Z(m, \cdot)], n \leq m
$$

Se $Z$ é um martingale a esperança de $Z(n, \cdot)$ é constante para todo $n \in \mathbb{N}$.

Exemplo 2.1. Sejam $X_{1}, \ldots, X_{n}$ variáveis independentes e identicamente distribuídas com $E\left(X_{i}\right)=0, i=1, \ldots, n$. Considere

$$
S(k, \omega)=\sum_{i=1}^{k} X_{i}(\omega), \quad k \in\{1, \ldots, n\} \text { e } \omega \in\left(S^{\infty}\right)^{n} .
$$

Definimos a filtragem interna $\left\{\mathcal{F}_{k}\right\}$

$$
\begin{aligned}
\mathcal{F}_{0} & =\left\{\emptyset,\left(S^{\infty}\right)^{n}\right\} \\
\mathcal{F}_{1} & =\text { álgebra gerada por }\left\{X_{1}\right\} \\
\mathcal{F}_{2} & =\text { álgebra gerada por }\left\{X_{1}, X_{2}\right\} \\
& \vdots \\
\mathcal{F}_{k} & =\text { álgebra gerada por }\left\{X_{1}, X_{2}, \ldots, X_{k}\right\} .
\end{aligned}
$$

Com isso $S$ é um processo estocático adaptado para a filtragem $\mathcal{F}_{k}$ e, mais ainda, $S$ é um martingale. De fato,

$$
\begin{aligned}
E\left[S(k+1, \cdot) \mid \mathcal{F}_{k}\right] & =E\left[X_{k+1}+S(k, \cdot) \mid \mathcal{F}_{k}\right] \\
& =E\left[X_{k+1} \mid \mathcal{F}_{k}\right]+S(k, \cdot)=E\left[X_{k+1}\right]+S(k, \cdot)=S(k, \cdot) .
\end{aligned}
$$

O processo de contagem $N$ definido em (2.3) tem tem trajetórias não descrescentes. Assim, verificamos facilmente que $N$ é um submartingale com relação à filtragem interna $\mathcal{A}_{n}^{N}$. De fato,

$$
\begin{aligned}
E\left[N(i+1, .) \mid \mathcal{A}_{i}^{N}\right] & =E\left[N(i+1, .)-N(i, .)+N(i, .) \mid \mathcal{A}_{i}^{N}\right] \\
& =E\left[N(i+1, .)-N(i, .) \mid \mathcal{A}_{i}^{N}\right]+E\left[N(i, .) \mid \mathcal{A}_{i}^{N}\right] \geq N(i, .)
\end{aligned}
$$

para $i=1, \ldots, n$. 
Proposição 2.1. Seja $Z=\left\{Z(n,):. n \in \mathbb{N}_{0}\right\}$ um martingale com respeito a filtragem $\mathcal{E}_{n} e$ $k \in \mathbb{N}$ tal que $1 \leq k \leq n$. Então, para $n>p$,

$$
E\left[Z^{2}(n, .)\right]-E\left[Z^{2}(p, .)\right]=\sum_{j=p}^{n-1} E\left[(Z(j+1, .)-Z(j, .))^{2}\right]
$$

Demonstração. Seja $e_{j}=Z(j+1,)-.Z(j,$.$) . Então, para m \leq j$,

$$
\begin{aligned}
E\left(e_{j} \mid \mathcal{E}_{m}\right) & =E\left[E\left(Z(j+1, .)-Z(j, .) \mid \mathcal{E}_{j}\right) \mid \mathcal{E}_{m}\right] \\
& =E\left[-Z(j, .)+E\left(Z(j+1, .) \mid \mathcal{E}_{j}\right) \mid \mathcal{E}_{m}\right] \\
& =E\left[-Z(j, .)+Z(j, .) \mid \mathcal{E}_{m}\right]=0
\end{aligned}
$$

Podemos escrever $Z(n,)=.Z(p,)+.\sum_{j=p}^{n-1} e_{j}$. Assim,

$$
E\left[Z^{2}(, .)\right]=E\left[Z^{2}(p, .)\right]+2 \sum_{j=p}^{n-1} E\left[Z(p, .) e_{j}\right]+E\left[\left(\sum_{j=p}^{n-1} e_{j}\right)^{2}\right]
$$

Mas,

$$
\left(\sum_{j=p}^{n-1} e_{j}\right)^{2}=\sum_{j=p}^{n-1} e_{j}^{2}+\sum_{j, j^{\prime} j \neq j^{\prime}} e_{j} e_{j^{\prime}}
$$

Logo,

$$
E\left[Z^{2}(n, .)\right]=E\left[Z^{2}(p, .)\right]+\sum_{j=p}^{n-1} E\left(e_{j}^{2}\right)+\sum_{j, j^{\prime}} E\left(e_{j} e_{j^{\prime}}\right)+2 \sum_{j=p}^{n-1}+2 \sum_{j=p}^{n-1} E\left[Z(p, .) e_{j}\right]
$$

Vamos mostrar que todos os termos que aparecem nas últimas duas somas são zero. Assuma que $j<j^{\prime}$. Então,

$$
E\left(e_{j} e_{j}^{\prime}\right)=E\left(E\left(e_{j} e_{j}^{\prime}\right) \mid \mathcal{E}_{j+1}\right)=e_{j} E\left(e_{j} \mid \mathcal{E}_{j+1}\right)
$$

Como $j \leq j^{\prime}$, temos, por 2.4, que $E\left(e_{j^{\prime}}\right)=0$, então $E\left(e_{j} e_{j}^{\prime} \mid \mathcal{E}_{j+1}\right)=0$. De forma análoga, obtemos que $E\left[Z(p,.) e_{j}\right]=E\left[E\left(Z(p,.) e_{j} \mid \mathcal{E}_{j}\right)\right]=0$. 


\subsubsection{Martingale Array Difference}

Nesta seção apresentamos a definição de martingale array difference e propriedades relacionadas. Estas informações serão necessárias para o estudo de convergência realizado no Capítulo 4.

Definição 2.4. Se $Z=\left\{Z(n,):. n \in \mathbb{N}_{0}\right\}$ é um processo estocástico adaptado a filtragem $\left\{\mathcal{E}_{n}\right\}$ com

$$
E\left[Z(n, .) \mid \mathcal{E}_{n-1}\right]=0 \text { para } n \geq 1,
$$

então, $Z$ é um martingale array difference.

Consideremos a soma parcial $U(n,)=.\sum_{j=1}^{n} Z(j,$.$) . O seguinte resultado é válido.$

Proposição 2.2. Seja $\left\{U(n,):. n \in \mathbb{N}_{0}\right\}$ um processo estocástico adptado à filtragem $\left\{\mathcal{E}_{n}\right\}$. Seja $Z(0,)=$.0 e $Z(n,)=.U(n,)-.U(n-1,$.$) para n \leq 1$. Então, o processo $\left\{U(n,):. n \in \mathbb{N}_{0}\right\}$ é um martingale se, e somente se, $\left\{Z(n,):. n \in \mathbb{N}_{0}\right\}$ é um martingale array difference.

Demonstração. Temos que $Z(n,)=.U(n,)-.U(n-1,$.$) . Então, Z(n,$.$) é um martingale array$ difference se, e somente se,

$$
E\left[Z(n, .) \mid \mathcal{E}_{n-1}\right]=E\left[U(n, .) \mid \mathcal{E}_{n-1}\right]-U(n-1,)=0 .
$$

Mas $E\left[U(n,) \mid. \mathcal{E}_{n-1}\right]-U(n-1)=$,0 se, e somente se, $U(n,$.$) é um martingale.$

\subsection{Integral Estocástica}

Nesta seção, introduzimos o conceito de integral estocástica, fundamental para o estudo de propriedades de estimadores. Tomemos $\left(S^{\infty}, \mathcal{A}, P\right)$ um espaço de probabilidade, $\left\{\mathcal{E}_{n}\right\}$ uma filtragem e denotemos por $\left(S^{\infty}, \mathcal{A},\left\{\mathcal{E}_{n}\right\}, P\right)$ a base estocástica associada. Um processo estocástico $Z$ está definido sobre a base estocástica $\left(S^{\infty}, \mathcal{A},\left\{\mathcal{E}_{n}\right\}, P\right)$ se $Z(n,$.$) são variáveis aleatórias$ definidas sobre $\left(S^{\infty}, \mathcal{A}, P\right)$ e $Z$ é adaptado para a filtragem $\left\{\mathcal{E}_{n}\right\}$.

Considere $Y$ e $V$ dois processos definidos na base estocástica $\left(S^{\infty}, \mathcal{A},\left\{\mathcal{E}_{n}\right\}, P\right)$. Definimos a integral estocástica como sendo

$$
(V . Y)(n, \cdot)=V(0, \cdot) Y(0, \cdot)+\sum_{i=1}^{n} V(i, \cdot) \Delta Y(i, \cdot)
$$

no qual $\Delta Y(i, \cdot)=Y(i, \cdot)-Y(i-1, \cdot)$, para todo $n \in \mathbb{N}$. 
Teorema 2.2. Seja $Y$ um martingale e $V$ um processo estocástico, ambos definidos na base estocástica $\left(S^{\infty}, \mathcal{A},\left\{\mathcal{E}_{n}\right\}, P\right)$. Então, as seguintes afirmações são equivalentes:

(i) O processo $V$ é previsivel, isto é, $V(n,.) \in S\left(\mathcal{E}_{n-1}\right)$.

(ii) A integral estocástica (V.Y) é martingale.

Demonstração. Consideremos $Y$ um martingale e $V$ um processo previsível. Assim, temos que

$$
\begin{aligned}
E\left[(V . Y)(n+1, .) \mid \mathcal{E}_{n}\right] & =E\left[V(0, .) . Y(0, .)+\sum_{i=1}^{n} V(i, .) \Delta Y(i, .)+V(n+1, .) \Delta Y(n+1, .) \mid \mathcal{E}_{n}\right] \\
& =V(0, .) Y(0, .)+\sum_{i=1}^{n} V(i, .) \Delta Y(i, .)+E\left[V(n+1, .) \Delta Y(n+1, .) \mid \mathcal{E}_{n}\right] \\
& =(V . Y)(n, .)+E\left[V(n+1) \Delta Y(n+1, .) \mid \mathcal{E}_{n}\right] \\
& =(V . Y)(n, .)+V(n+1) E\left[\Delta Y(n+1, .) \mid \mathcal{E}_{n}\right] \\
& =(V . Y)(n, .)+V(n+1) E\left[Y(n+1, .) \mid \mathcal{E}_{n}\right]-Y(n, .) \\
& =(V . Y)(n, .)+V(n+1)[Y(n, .)-Y(n, .)]=(V . Y)(n, .)
\end{aligned}
$$

Então, $E\left[(V . Y)(n+1,) \mid. \mathcal{E}_{n}\right]=(V . Y)(n,$.$) , ou seja, (V . Y)$ é uma martingale.

Por outro lado, como

$$
E\left[(V . Y)(n+1, .) \mid \mathcal{E}_{n}\right]=(V . Y)(n, .)+E\left[V(n+1) \Delta Y(n+1, .) \mid \mathcal{E}_{n}\right]
$$

e $(V . Y)$ é um martingale, $E\left[V(n+1) \Delta Y(n+1,) \mid. \mathcal{E}_{n}\right]$ deve ser igual a zero. No entanto,

$$
E\left[V(n+1) \Delta Y(n+1, .) \mid \mathcal{E}_{n}\right]=0 \Leftrightarrow V(n+1) \text { e } \Delta Y(n+1, .) \text { são independentes. }
$$

Para realizar esta demonstração consideramos o fato de $\Delta Y(n+1,$.$) ser \mathcal{A}_{n+1^{-}}$mensurável e $V(n,.) \operatorname{ser} \mathcal{A}_{n^{-}}$mensurável.

Da mesma forma, mostramos que se $Y$ é um supermartingale (submartingale) e $V$ é um processo previsível, então, a integral estocástica ( $V . Y)$ é um supermartingale (submartingale). 


\subsection{Decomposição de Doob-Meyer}

A decomposição de Doob-Meyer é um resultado fundamental na teoria dos processos estocásticos, pois garante que um processo estocástico possa ser decomposto como a soma de um martingale e um processo previsível. Este fato permite a decomposição do processo de contagem $N$, associado a uma variável aleatória $X$, em um componente ruído (martingale) e um componente estimável (previsível). Utilizando deste componente previsível, vamos estabelecer o modelo de intensidade mltiplicativo.

Teorema 2.3. Seja $Z: \mathbb{N}_{0} \times S^{\infty} \rightarrow \mathbb{R}$ um processo estocástico definido na base estocástica $\left(S^{\infty}, \mathcal{A},\left\{\mathcal{E}_{n}\right\}, P\right)$ com $Z(0,)=$.0 . O processo $Z$ pode ser decomposto de forma única em

$$
Z=Y+A
$$

no qual $Y$ é um martingale e A um processo previsivel, satisfazendo

$$
Y(n, .)=Z(0, .)+\sum_{l=1}^{n}\left[Z(l, .)-E\left(Z(l, .) \mid \mathcal{E}_{l-1}\right)\right], n \geq 1
$$

$e$

$$
A(n, .)=\sum_{l=1}^{n}\left[E\left[Z(l, .) \mid \mathcal{E}_{l-1}\right]-Z(l-1, .)\right], n \geq 1
$$

$\operatorname{com} Y(0,)=.Z(0,)=$.0 e $A(0,)=$.0 .

Demonstração. (i) Temos que $Z=Y+A$ :

$$
\begin{aligned}
Z(0, .) & =Y(0, .)+A(0, .) \\
Z(1, .) & =Z(0, .)+Z(1, .)-E\left[Z(1, .) \mid \mathcal{E}_{0}\right]+E\left[Z(1, .) \mid \mathcal{E}_{0}\right]-Z(0, .) \\
& =Y(1, .)+A(1, .) \\
Z(2, .) & =Z(0, .)+Z(1, .)-E\left[Z(1, .) \mid \mathcal{E}_{0}\right]+E\left[Z(1, .) \mid \mathcal{E}_{0}\right]-Z(0, .) \\
& +Z(2, .)-E\left[Z(2, .) \mid \mathcal{E}_{1}\right]+E\left[Z(2, .) \mid \mathcal{E}_{1}\right]-Z(1, .) \\
& =Y(2, .)+A(2, .)
\end{aligned}
$$

Assim, de forma indutiva,

$$
Z(n, .)=Y(n, .)+A(n, .) .
$$


(ii) Da a equação 2.7, podemos ver que $Y$ é $\mathcal{A}_{n}$-mensuráel. Além disso,

$$
\begin{aligned}
E\left[Y(n, .) \mid \mathcal{E}_{n-1}\right] & =Z_{0}+\sum_{l=1}^{n-1}\left[Z(l, .)-E\left[Z(l, .) \mid \mathcal{E}_{l-1}\right]\right]+E\left[Z(n, .) \mid \mathcal{E}_{n-1}\right]-E\left[Z(n, .) \mid \mathcal{E}_{n-1}\right] \\
& =Y(n-1, .)
\end{aligned}
$$

Portanto $Y$ é um martingale.

Observando a equação (2.8), verificamos que $A$ é um processo previsível.

(iii) Considere, agora, que $Z$ tem duas representações

$$
Z=Y+A \quad \text { e } \quad Z=Y^{\prime}+A^{\prime}
$$

com $Y$ e $Y^{\prime}$ martingales e $A$ e $A^{\prime}$ processos previsíveis. Então,

$A^{\prime}(n+1,)-.A^{\prime}(n,)=.[A(n+1,)-.A(n,)]-.[Y(n+1,)-.Y(n,)]+.\left[Y^{\prime}(n+1,)-.Y^{\prime}(n,).\right]$.

Agora,

$$
\begin{aligned}
E\left[A^{\prime}(n+1, .)-A^{\prime}(n, .) \mid \mathcal{E}_{n}\right] & =E\left[A(n+1, .)-A(n, .) \mid \mathcal{E}_{n}\right]+E\left[Y(n+1, .)-Y(n, .) \mid \mathcal{E}_{n}\right] \\
& +E\left[Y^{\prime}(n+1, .)-Y^{\prime}(n, .) \mid \mathcal{E}_{n}\right]
\end{aligned}
$$

Mas, observe que, como $Y$ é um martingale temos que

$$
E\left[Y(n+1, .)-Y(n, .) \mid \mathcal{E}_{n}\right]=-Y(n, .)+E\left[Y(n+1, .) \mid \mathcal{E}_{n}\right]=-Y(n, .)+Y(n, .)=0
$$

Da mesma forma, como $Y^{\prime}$ também é um martingale, obtemos que $E\left[Y^{\prime}(n+1,)-\right.$. $\left.Y^{\prime}(n,) \mid. \mathcal{E}_{n}\right]=0$. Assim,

$$
E\left[A^{\prime}(n+1, .)-A^{\prime}(n, .) \mid \mathcal{E}_{n}\right]=E\left[A(n+1, .)-A(n, .) \mid \mathcal{E}_{n}\right]
$$

Como $A$ e $A^{\prime}$ são previsíveis

$$
A^{\prime}(n+1, .)-A^{\prime}(n, .)=A(n+1, .)-A(n, .) \Rightarrow A^{\prime}=A
$$

Assim, temos também que $Y^{\prime}=Y$. Portanto, a decomposição de Doob é única. 


\subsubsection{Processo Variação Quadrática}

O processo variação quadrática corresponde a uma das principais aplicações da decomposição de Doob-Meyer. Dado um martingale $Z$ com respeito a base estocástica $\left(S^{\infty}, \mathcal{A},\left\{\mathcal{E}_{n}\right\}, P\right)$, o processo variação quadrática associado a $Z$ pode ser interpretado como sua volatilidade, e será utilizado para calcularmos a variância de um martingale. Desde que $Z$ é um martingale, obtemos da desigualdade de Jensen que $Z^{2}$ é um submartingale. Na realidade,

$$
\begin{aligned}
E\left[Z^{2}(n+1, .) \mid \mathcal{E}_{n}\right] & \geq\left(E\left[Z(n+1, .) \mid \mathcal{E}_{n}\right]\right)^{2} \\
& =Z^{2}(n+1, .) .
\end{aligned}
$$

Pela decomposição de Doob-Meyer, existe um único processo previsível crescente denotado por $<Z, Z>$ tal que $Z^{2}-<Z, Z>$ é um martingale. O processo $<Z, Z>$ é denominado variação quadrática (ou compensador) e é definido como

$$
<Z, Z>=\sum_{l=1}^{i} E\left[(\Delta Z(l, .))^{2} \mid \mathcal{E}_{l-1}^{n}\right], \quad i \leq k .
$$

Ao considerar $X$ uma variável aleatória, $N(i,)=.\mathbb{1}_{\{X \leq i\}}$ o processo de contagem associado a $X$ com sua filtragem interna, sabemos que

$$
N(i, .)=Y(i, .)+A(i, .)
$$

com $Y$ um martingale e $A$ um processo previsível crescente.

Na seqüência, vamos calcular o processo variação quadrática de $Y$. Inicicalmente, temos que

$$
[Y(i, .)]^{2}=2 \sum_{l=1}^{i} Y(l-1, .) \Delta Y(l, .)+\sum_{l=1}^{i}[\Delta Y(l, .)]^{2}
$$

Além disso,

$$
[\Delta Y(i, .)]^{2}=[\Delta N(i, .)-\Delta A(i, .)]^{2}=[\Delta N(i, .)]^{2}-2 \Delta N(i, .) \Delta A(i, .)+[\Delta A(i, .)]^{2}
$$


Assim,

$$
\begin{aligned}
{[Y(i, .)]^{2} } & =2 \sum_{l=1}^{i} Y(l-1, .) \Delta Y(l, .)+\sum_{l=1}^{i}[\Delta N(l, .)]^{2}-2 \sum_{l=1}^{i} \Delta N(l, .) \Delta A(l, .)+\sum_{l=1}^{i}[\Delta A(l, .)]^{2} \\
& =2 \sum_{l=1}^{i} Y(l-1, .) \Delta Y(l, .)+\sum_{l=1}^{i} \Delta N(l, .)-2 \sum_{l=1}^{i} \Delta N(l, .) \Delta A(l, .)+\sum_{l=1}^{i}[\Delta A(l, .)]^{2} \\
& =2 \sum_{l=1}^{i} Y(l-1, .) \Delta Y(l, .)+\sum_{l=1}^{i} \Delta Y(l, .)+\sum_{l=1}^{i} \Delta A(l, .) \\
& -2 \sum_{l=1}^{i} \Delta A(l, .) \Delta Y(l, .)+\sum_{l=1}^{i} \Delta A(l, .) \Delta A(l, .)-2 \sum_{l=1}^{i} \Delta A(l, .) A(l, .) \\
& =2 \sum_{l=1}^{i} Y(l-1, .) \Delta Y(l, .)+\sum_{l=1}^{i} \Delta Y(l, .)+\sum_{l=1}^{i} \Delta A(l, .) \\
& -2 \sum_{l=1}^{i} \Delta A(l, .) \Delta Y(l, .)-\sum_{l=1}^{i} \Delta A(l, .) \Delta A(l, .) \\
& =\sum_{l=1}^{i}[2 Y(l-1, .)+1-2 \Delta A(l, .)] \Delta Y(l, .)+\sum_{l=1}^{i}[1-\Delta A(l, .)] \Delta A(l, .) \\
& =(G . Y)(i, .)+((1-\Delta A) . A)(i, .),
\end{aligned}
$$

no qual $G(l,)=.2 Y(l-1,)+1-.2 \Delta A(l,$.$) para l=1, \cdots, k$. Desde que $G$ é previsível, obtemos a decomposição de $Y^{2}$ em um martingale mais um processo previsível crescente. Assim, como a decomposição de Doob-Meier é única, obtemos que a variação quadrática é dada por

$$
<Y, Y>(i, .)=\sum_{l=1}^{i}[1-\Delta A(l, .)] \Delta A(l, .)=[(1-\Delta A) . A](i, .)
$$

Proposição 2.3. Sejam $Z$ um martingale e $V$ um processo previsível com respeito a base estocástica $\left(S^{\infty}, \mathcal{A},\left\{\mathcal{E}_{n}\right\}, P\right)$. Assim, temos que

$$
<(V . Z),(V . Z)>=\left(V^{2} \cdot<Z, Z>\right)
$$


Demonstração.

$$
\begin{aligned}
<(V . Z),(V . Z)>(i, .) & =\sum_{l=1}^{i} E\left[(\Delta(V . Z)(l, .))^{2} \mid \mathcal{E}_{l-1}\right]=\sum_{l=1}^{i} E\left[(V(l, .) \Delta Z(l, .))^{2} \mid \mathcal{E}_{l-1}\right] \\
& =\sum_{l=1}^{i} E\left[V^{2}(l, .)\left(\Delta Z(l, .)^{2}\right) \mid \mathcal{E}_{l-1}\right]=\sum_{l=1}^{n} V^{2}(l, .) E\left[(\Delta Z(l, .))^{2} \mid \mathcal{E}_{l-1}\right] \\
& =\sum_{l=1}^{i} V^{2}(l, .) \Delta<Z, Z>(l, .)=\left(V^{2} .<Z, Z>\right)(i, .) .
\end{aligned}
$$




\section{Capítulo 3}

\section{Modelo de Intensidade Multiplicativo}

\subsection{Introdução}

O modelo de intensidade multiplicativo foi proposto por Aalen (1978) para o caso de variáveis aleatórias com distribuição de probabilidade absolutamente contínua. Para uma descrição detalhada deste modelo ver Fleming e Harrington (1990). Neste trabalho fizemos uma adaptação do modelo de intensidade multiplicativo para tratar o caso de variáveis aleatórias à valores em um espaço finito que é introduzido neste capítulo.

Para um maior entendimento das definições apresentadas e hipóteses assumidas neste capítulo, utilizamos como motivação os dados de alunos de graduação da Universidade de São Paulo apresentados na Seção 1.1.

Na segunda seção apresentamos o modelo de intensidade multiplicativo para variáveis aleatórias multinomais. Na terceira seção introduzimos o estudo de inferência no modelo de intensidade multiplicativo, obtemos os estimadores dos parâmetros do modelo e estudamos as propriedades finitas e assintóticas desses estimadores.

\subsection{Desenvolvimento do Modelo}

Consideremos $W$ e $C$ variáveis aleatórias multinomiais independentes assumindo valores $\{1, \cdots, k, k+$ $1\}, k \in \mathbb{N}$. Aos valores assumidos por essas variáveis nos referimos como categorias ou classes. Quando a variável $W$ assumir valores $\{1, \ldots, k\}$ ela representa a categoria em que ocorreu o 
evento de interesse. Caso o evento de interesse não tenha ocorrido, $W$ assume valor $k+1 \mathrm{e}$ a variável $C$ representa a categoria em que ocorreu o evento de censura. De forma resumida temos que

$$
\begin{aligned}
& W= \begin{cases}i, & \text { se ocorreu o evento de interesse na categoria } i, i=1, \ldots, k ; \\
k+1, & \text { se não ocorreu o evento de interesse até a categoria } k .\end{cases} \\
& C= \begin{cases}i, & \text { se ocorreu o evento de censura na categoria } i, i=1, \ldots, k ; \\
k+1, & \text { se não ocorreu evento de censura até a categoria } k .\end{cases}
\end{aligned}
$$

Definimos a variável multinomial $X$ como sendo o mínimo entre $W$ e $C$,

$$
X=W \wedge C .
$$

Denotamos por $\eta$ a distribuição de probabilidade associada à variável aleatória $X$ e por $\zeta$ à distribuição de probabilidade associada à variável $W$.

Consideremos o conjunto de dados apresentado na Seção 1.1. Associamos a cada aluno a variável aleatória multinomial $X$ que representa o número de semestres até a última ocorrência. O evento de interesse é ter como última ocorrência evasão. Se o aluno evadiu no semestre $i$, $C=k+1$ e $X=W=i$, para $i=1, \ldots, k$. Se o aluno migrou, faleceu ou concluiu no semestre $i$, eventos considerados de censura, $W=k+1$ e $X=C=i$, para $i=1, \ldots, k$. Se o aluno estiver em andamento até o fim do estudo, $X=W=C=k+1$.

Observe que o valor $k$ é o número de semestres analisados. Por exemplo, ao estudarmos os alunos ingressantes em 2004, teremos no máximo 10 semestres para serem considerados. Neste caso, $k=10$ e, portanto, $C$ e $W$ assumem o valor 11 se o aluno estiver ativo no fim do estudo.

Em notação de eventos, como a evasão é o evento de interesse na análise dos alunos de graduação da USP, o evento $\{X=i, X=W\}$ determina a evasão do aluno no semestre $i$, para $i=1, \ldots, k$, o evento $\{X=i, X=C\}$ determina se o aluno migrou, faleceu ou concluiu no semestre $i, i=1, \ldots, k$, e o evento $\{X=k+1, X=W=C\}$ determina se o aluno está ativo até o semestre $k$.

Definimos o processo estocástico $N:\{0,1,2, \cdots, k\} \times S^{\infty} \rightarrow\{0,1\}$ conforme a equação 3.1

$$
N(i, .)=\mathbb{1}_{\{X \leq i, X=W\}}, \quad i=1, \cdots, k .
$$


O processo $N$ é um processo de contagem, com trajetórias não decrescentes e a variável aleatória $N(i,$.$) determina se o evento de interesse ocorreu até a categoria i$. Definimos a álgebra $\mathcal{B}_{i}^{N}$ associada ao processo $N$ conforme

$$
\begin{aligned}
\mathcal{B}_{0}^{N} & =\text { álgebra gerada por }\left\{\emptyset, S^{\infty}\right\} \\
\mathcal{B}_{1}^{N} & =\text { álgebra gerada por }\{N(1, .), C\} \\
\mathcal{B}_{2}^{N} & =\text { álgebra gerada por }\{N(1, .), N(2, .), C\} \\
& \vdots \\
\mathcal{B}_{i}^{N} & =\text { álgebra gerada por }\{N(1, .), N(2, .), N(3, .), \ldots, N(i, .), C\}
\end{aligned}
$$

Denotamos por $\left\{\mathcal{B}^{N}\right\}$ a filtragem interna associada ao processo $N$ formada pelas álgebras encaixantes $\mathcal{B}_{i}^{N}, 0 \leq i \leq k$.

Pela decomposição de Doob-Meyer 2.8, sabemos que o processo de contagem $N$ pode ser representado por

$$
N(i, .)=Y(i, .)+A(i, .)
$$

para $i=1, \cdots, k$ no qual $Y$ é martingale e $A$ é um processo previsível com respeito a base estocástica $\left(S^{\infty}, \mathcal{A},\left\{\mathcal{B}^{N}\right\}, P\right)$, com $A(0,)=$.0 e $Y(0,)=$.0 . Desde que $N$ é um submartingale, concluímos que $A$ tem trajetórias não decrescentes, satisfazendo

$$
\begin{array}{r}
Y(i, .)=\sum_{l=1}^{i}\left[N(l, .)-E\left[N(l, .) \mid \mathcal{B}_{l-1}^{N}\right]\right] \\
A(i, .)=\sum_{l=1}^{i}\left[E\left[N(l, .) \mid \mathcal{B}_{l-1}^{N}\right]-N(l-1, .)\right]
\end{array}
$$

$\operatorname{com} A(0,)=$.0 e $Y(0,)=$.0 . Na seqüência, são calculados os processos estocásticos relacionados com a decomposição de Doob-Meyer.

$$
\begin{aligned}
A(1, .) & =E\left[N(1, .) \mid \mathcal{B}_{0}^{N}\right]-N(0, .)=E[N(1, .)]=E\left[\mathbb{1}_{\{X \leq 1, X=W\}}\right]=P[W=1] \\
A(2, .) & =A(1, .)+E\left[N(2, .) \mid \mathcal{B}_{1}^{N}\right]-N(1, .) \\
& =P[W=1]+E\left[N(2, .)-N(1, .) \mid \mathcal{B}_{1}^{N}\right]=P[W=1]+E[N(2, .)-N(1, .) \mid N(1, .), C]
\end{aligned}
$$


Observe que se $N(1,)=$.1 , então

$$
N(2, .)-N(1, .)=\mathbb{1}_{\{X=2, X=W\}}=0
$$

e portanto, $E[N(2,)-.N(1,) \mid. N(1,), C]=$.0 . Se $N(1,)=$.0 e $C=1, E[N(2,)-.N(1,) \mid. N(1,), C]=$. 0. E, finalmente, se $N(1,)=$.0 e $C \geq 2, E[N(2,)-.N(1,) \mid. N(1,), C] \neq$.0 . Desde que $W$ e $C$ são independentes, concluímos que

$$
\begin{aligned}
E[N(2, .)-N(1, .) \mid N(1, .), C] & =E[N(2, .)-N(1, .) \mid N(1, .)=0, C \geq 2] \mathbb{1}_{\{N(1, .)=0, C \geq 2\}} \\
& =E\left[\mathbb{1}_{\{X \leq 2, X=W\}}-\mathbb{1}_{\{X \leq 1, X=W\}} \mid N(1, .)=0, C \geq 2\right] \mathbb{1}_{\{N(1, .)=0, C \geq 2\}} \\
& =E\left[\mathbb{1}_{\{W=2\}} \mid N(1, .)=0, C \geq 2\right] \mathbb{1}_{\{N(1, .)=0, C \geq 2\}} \\
& =\frac{P[W=2 ; N(1, .)=0 ; C \geq 2]}{P[N(1, .)=0 ; C \geq 2]} \mathbb{1}_{\{N(1, .)=0, C \geq 2\}} \\
& =\frac{P[W=2]}{P[W \geq 2]} \mathbb{1}_{\{N(1, .)=0, C \geq 2\}}
\end{aligned}
$$

Com isso,

$$
A(2, .)=A(1, .)+\frac{P[W=2]}{P[W \geq 2]} \mathbb{1}_{\{N(1, .)=0, C \geq 2\}} .
$$

Realizando cálculos análogos a estes, obtemos

$$
\begin{aligned}
A(k, .) & =A(k-1, .)+E\left[N(k, .) \mid \mathcal{A}_{k-1}^{N}\right]-N(k-1, .) \\
& =A(k-1, .)+E\left[N(k, .)-N(k-1, .) \mid \mathcal{A}_{k-1}^{N}\right] \\
& =A(k-1, .)+E[N(k, .)-N(k-1, .) \mid N(k-1, .)=0, C \geq k] \mathbb{1}_{\{N(k-1, .)=0, C \geq k\}} \\
& =A(k-1, .)+E\left[\mathbb{1}_{\{W=k\}} \mid N(k-1, .)=0, C \geq k\right] \mathbb{1}_{\{N(k-1, .)=0, C \geq k\}} \\
& =A(k-1)+\frac{P[W=k]}{P[W \geq k]} \mathbb{1}_{\{N(k-1, .)=0, C \geq k\}} .
\end{aligned}
$$

De forma geral, obtemos

$$
A(i, .)=\sum_{l=1}^{i} \frac{P[W=l]}{P[W \geq l]} \mathbb{1}_{\{N(l-1, .)=0, C \geq l\}}=\sum_{l=1}^{i} \frac{P[W=l]}{P[W \geq l]} \mathbb{1}_{\{X \geq l\}}
$$

para $i=1, \ldots, k+1$.

Finalmente, concluímos que

$$
Y(i, .)=N(i, .)-\sum_{l=1}^{i} \frac{P[W=l]}{P[W \geq l]} \mathbb{1}_{\{X \geq l\}} .
$$

para $i=1, \ldots, k+1$. 
Definição 3.1. A função intensidade $h:\{0,1,2, \cdots, k\} \rightarrow[0,1]$, associada ao processo de contagem $N$, é definida por

$$
h(i)=\frac{P[W=i]}{P[W \geq i]}, \quad i=1, \cdots, k,
$$

$\operatorname{com} h(0)=0$ e $h(k) \leq 1$.

A partir da definição a função intensidade $h$, obtemos que a distribuição de probabilidade associada à variável aleatória multinomial é dada por

$$
\begin{aligned}
\zeta(i)=P[W=i] & =[1-h(1)][1-h(2)] \ldots[1-h(i-1)] h(i, .) \\
& =h(i) \prod_{l=1}^{i-1}[1-h(l)] ; i=1 \cdots, k .
\end{aligned}
$$

De fato, pela equação 3.3 , temos que

$$
\begin{aligned}
h(1) & =P[W=1] \\
h(2) & =\frac{P[W=2]}{P[W \geq 2]}=\frac{P[W=2]}{1-P[W=1]} \\
& \Rightarrow P[W=2]=h(2)(1-P[W=1])=(1-h(1)) h(2) ; \\
h(3) & =\frac{P[W=3]}{P[W \geq 3]}=\frac{P[W=3]}{1-(P[W=1]+P[X=2])} \\
& \Rightarrow P[W=3]=h(3)[1-(P[W=2]+P[W=1])]=(1-h(1))(1-h(2)) h(3) .
\end{aligned}
$$

Por indução sobre $k$, obtemos que

$$
\begin{aligned}
h(k) & =\frac{P[W=k]}{P[W \geq k]}=\frac{P[W=k]}{1-\left(\sum_{l=1}^{k-1} P[W=l]\right)} \\
& \Rightarrow P[W=k]=h(k)\left[1-\sum_{l=1}^{k-1} P[W=l]\right] \\
& =h(k)[1-[(1-h(1)) \ldots(1-h(k-2)) h(k-1)]+\ldots+(1-h(1)) h(2)+h(1)] \\
& =h(k)(1-h(1))(1-h(2))(1-h(3)) \ldots(1-h(k-1))
\end{aligned}
$$

Assim, concluímos que a relação apresentada na equação 3.4 é válida.

Defininamos o processo previsível $V(i,)=.\mathbb{1}_{\{X \geq i\}}$, em que

$$
\{X \geq i\}=\{X \geq i, X=W\} \cup\{X \geq i, X=C\}, \quad i=1, \ldots, k
$$


Com isso, podemos denotar a distribuição de probabilidade $\zeta$ definda na equação 3.5 da seguinte maneira

$$
\zeta(i)=P[W=i]=\prod_{l=1}^{k}[1-h(i)]^{V(l, .)-\Delta N(l, .)}[h(i)]^{V(l, .)} .
$$

A partir da função intensidade, o processo previsível $A$ pode ser representado na forma

$$
A(i, .)=\sum_{l=1}^{i} h(l) \mathbb{1}_{\{X \geq l\}}=\sum_{l=1}^{i} V(l, .) \Delta H(l)
$$

no qual $H$ é a função intensidade acumulada, dada por $H(i)=\sum_{l=1}^{i} h(l)$. Assim, concluímos que o processo previsível $A$ pode ser representado na forma multiplicativa

$$
A(i, .)=\sum_{l=1}^{i} V(l, \cdot) \Delta H(l)=(V . H)(i, .)
$$

na qual $\Delta H(l)=H(l)-H(l-1)=h(l)$. Como $V$ é previsível, o processo $A$ é determinado pela função intensidade $h$ (ou, pela função intensidade acumulada $H$ ). Então, dado uma variável aleatória $W$, existe uma única função intensidade $h$ satisfazendo (3.3). A seguir, vamos mostrar que a recíproca também é válida.

Teorema 3.1. As seguintes afirmações são válidas:

a. Dado $W$ uma variável aleatória multinomial definida sobre $\left(S^{\infty}, \mathcal{A}, P\right)$ com distribuição de probabilidade $\zeta(i)=P[W=i]$. Então, existe uma única função $h:\{0,1,2, \cdots, k\} \rightarrow$ $[0,1]$, tal que

$$
h(i)=\frac{P[W=i]}{P[W \geq i]}
$$

$\operatorname{com} h(0)=0, i=1, \cdots, k$.

b. Dado uma função h definida no item (a.), existe uma única variável aleatória $W$ sobre $\left(S^{\infty}, \mathcal{A}, P\right)$ cuja distribuição de probabilidade é unicamente determinada pela função intensidade h e satisfaz

$$
\zeta(i)=P[W=i]=h(i) \prod_{l=1}^{i-1}[1-h(l)]=\prod_{l=1}^{k}[1-h(i)]^{V(l, .)-\Delta N(l, .)}[h(i)]^{V(l, .)}
$$

na qual $V(l,)=.\mathbb{1}_{\{X \geq l\}}$ e $N(i,)=.\mathbb{1}_{\{X \leq l, X=W\}}$, para todo $i=1, \cdots, k$. 
Demonstração. Basta aplicarmos o Teorema 2.1.

Com este Teorema, relacionamos (de forma única) a classe de variáveis aleatórias $W$ e classe de funções intensidades $h$. Com isso, ao desenvolvermos um método para estimar a função intensidade $h$, estimamos também a distribuição de probabilidade $\zeta$.

\subsection{Inferência no Modelo de Intensidade Multiplicativo}

Consideremos uma amostra aleatória simples

$$
\left(\left(W_{1}^{(p)}, C_{1}^{(p)}\right), \ldots,\left(W_{n_{p}}^{(p)}, C_{n_{p}}^{(p)}\right)\right)
$$

para $p=1, \ldots, J$ e $n_{p}, J \in \mathbb{N}$. Além disso, consideremos que existe indpendência entre as variáveis $W_{m}^{(p)}$ e $C_{m}^{(p)}$, para $\forall m=1, \ldots, n_{p}$.

As variáveis $W_{m}^{(p)}$ e $C_{m}^{(p)}$ são variáveis aleatórias multinomiais assumindo valores $\{1, \ldots, k+$ 1\}. A variável $W_{m}^{(p)}$ representa a categoria de ocorrência do evento de interesse para o item $m$ da amostra $p$ quando assumir valores $1, \ldots, k$. Se $W_{m}^{(p)}=k+1$, o evento de interesse não ocorreu para o item $m$ da amostra $p$ e, portanto, a variável $C_{m}^{(p)}$ representa a categoria em que ocorreu o evento de censura para este item. Denotamos por $\zeta^{(p)}:\{0,1, \ldots, k+1\} \rightarrow[0,1]$ a distribuição de probabilidade associda à variável $W_{m}^{(p)}, m=1, \ldots, n_{p}$.

De forma análoga, definimos a variável $X_{m}^{(p)}$ como sendo o mínimo entre as variáveis $W_{m}^{(p)}$ e $C_{m}^{(p)}$, coforme a equação 3.8 ,

$$
X_{m}^{(p)}=W_{m}^{(p)} \wedge C_{m}^{(p)}
$$

tendo $X_{m}^{(p)}, m=1, \ldots, n_{p}$, distribuição de probabilidade $\eta^{(p)}:\{0,1, \ldots, k+1\} \rightarrow[0,1]$.

Em relação ao conjunto de dados referente aos alunos de graduação da USP apresentado na Seção 1.1, temos que $p$ corresponde ao ano de ingresso do aluno, $n_{p}$ corresponde ao número de ingressantes no ano $p$ e cada uma das variáveis da amostra $X^{(p)}$ está associada a cada um dos alunos que ingressou no ano $p$, representando o semestre de última ocorrência do aluno.

O evento $\left\{X_{m}^{(p)}=i, X_{m}^{(p)}=W_{m}^{(p)}\right\}$ representa se o aluno $m$ ingressante no ano $p$, para $m=1, \ldots, n_{p}$ e $p=1, \ldots, J$, evadiu até o semestre $i$, para $i=1, \ldots, k$. Se $W_{m}^{(p)}=k+1$, então o aluno $m$ ingressante no ano $p$ migrou, faleceu, concluiu ou ainda está ativo. Para 
identificar qual desses possíveis eventos ocorreu, utilizamos os eventos $\left\{X_{m}^{(p)}=i, X_{m}^{(p)}=C_{m}^{(p)}\right\}$ e $\left\{X_{m}^{(p)}=k+1, X_{m}^{(p)}=C_{m}^{(p)}\right\}$, que indicam que o aluno faleceu, migrou ou concluiu no semestre $i$ e que o aluno ainda está ativo ao fim do estudo, respectivamente.

Definimos o processo associado à variável aleatória $X_{m}^{(p)}$

$$
N_{m}^{(p)}(i, .)=\mathbb{1}_{\left\{X_{m}^{(p)} \leq i, X_{m}^{(p)}=W_{m}^{(p)}\right\}}, m=1, \cdots, n_{p}
$$

Este processo indica se o evento de interesse ocorreu para o item $m$ até a categoria $i$. Definimos também o processo de contagem $N^{n_{p}}$ associado a amostra aleatória $X^{(p)}$ conforme a equação 3.10

$$
N^{n_{p}}(i, .)=\sum_{m=1}^{n_{p}} N_{m}^{(p)}(i, .)
$$

A variável aleatória $N^{n_{p}}(i,$.$) indica quantos vezes o evento de interesse ocorreu para a amostra$ $X^{(p)}$ até a categoria $i, i=1, \ldots, k$. Desde que $N^{n_{p}}$ é soma de processos de contagem, concluímos que $N^{n_{p}}$ é um submartingale definido sobre $\{1,2, \cdots, k\} \times\left(S^{\infty}\right)^{n_{p}}$ com valores em $\left\{0,1,2, \cdots, n_{p}\right\}$.

Considerando os dados dos alunos de graduação da USP, temos que as variáveis aleatórias $N_{m}^{(p)}(i,$.$) e N^{n_{p}}(i,$.$) indicam, respectivamente, se o aluno m$ evadiu até o semestre $i$ e quantos alunos ingressantes no ano $p$ evadiram até o semestre $i$.

A seguir definimos as álgebras encaixantes $\mathcal{B}_{i}^{N^{n_{p}}}$, para $i=1, \ldots, k$,

$$
\begin{aligned}
\mathcal{B}_{0}^{N^{n_{p}}} & =\text { álgebra gerada por }\left\{\emptyset, S^{\infty}\right\} \\
\mathcal{B}_{1}^{N^{n_{p}}} & =\text { álgebra gerada por }\left\{N^{n_{p}}(1, .), C_{1}^{(p)}, \ldots, C_{n_{1}}^{(p)}\right\} \\
& \vdots \\
\mathcal{B}_{k}^{N^{n_{p}}} & =\text { álgebra gerada por }\left\{N^{n_{p}}(1, .), \ldots, N^{n_{p}}(k, .), C_{1}^{(p)}, \ldots, C_{n_{p}}^{(p)}\right\} .
\end{aligned}
$$

A filtragem interna, $\left\{\mathcal{B}^{N^{n_{p}}}\right\}$, associada ao processo $N^{n_{p}}$ é a união das álgebras encaixantes $\mathcal{B}_{i}^{N^{n_{p}}}, 0 \leq i \leq k$. Pela decomposição de Doob-Meyer, o processo de contagem $N^{n_{p}}$ pode ser representado da seguinte forma

$$
N^{n_{p}}(i, .)=Y^{n_{p}}(i, .)+A^{n_{p}}(i, .)
$$


no qual $A^{n_{p}}$ é um processo previsível com trajetórias não decrescentes, $Y^{n_{p}}$ é um martingale, $\operatorname{com} A^{n_{p}}(0,)=.Y^{n_{p}}(0,)=$.0 , dados respectivamente por

$$
\begin{array}{r}
A(i, .)=\sum_{l=1}^{i}\left[E\left[N(l, .) \mid \mathcal{B}_{l-1}^{N}\right]-N(l-1, .)\right] \\
Y(i, .)=\sum_{l=1}^{i}\left[N(l, .)-E\left[N(l, .) \mid \mathcal{B}_{l-1}^{N}\right]\right] .
\end{array}
$$

Na seção anterior, foram calculados os processos $A$ e $Y$ para uma única variável. Realizando cálculos análogos para o caso de uma amostra, obtemos que

$$
A^{n_{p}}(i, .)=\sum_{l=1}^{i} \sum_{m=1}^{n_{p}} \mathbb{1}_{\left\{X_{m}^{(p)} \geq l\right\}} h^{(p)}(l),
$$

em que

$$
h^{(p)}(i)=\frac{P\left[W_{m}^{(p)}=i\right]}{P\left[W_{m}^{(p)} \geq i\right]},
$$

é a função intensidade associada ao processo de contagem $N^{n_{p}}(i,$.$) , para i=1, \ldots, k$ e $\forall m, p$, com $m=1, \ldots, n_{p}$ e $p=1, \ldots, J$. Conseqüentemente, obtemos que

$$
Y^{n_{p}}(i, .)=N^{n_{p}}(i, .)-\sum_{l=1}^{i} \sum_{m=1}^{n_{p}} \mathbb{1}_{\left\{X_{m}^{(p)} \geq l\right\}} h^{(p)}(l)
$$

Consideremos o processo previsível $V^{n_{p}}$ e a função intensidade acumulada $H^{(p)}$ dados, respectivamente, por

$$
\begin{aligned}
V^{n_{p}}(l, .) & =\sum_{m=1}^{n_{p}} \mathbb{1}_{\left\{X_{m}^{(p)} \geq l\right\}} \\
H^{(p)}(i) & =\sum_{l=1}^{i} h^{(p)}(l) .
\end{aligned}
$$

Com isso, obtemos o modelo de intensidade multiplicativo

$$
\left.A^{n_{p}}(i, .)=\sum_{l=1}^{i} V^{n_{p}}(l, .) h^{(p)}(l)=\sum_{l=1}^{i} V^{n_{p}}(l, .) \Delta H^{(} p\right)=\left(V^{n_{p}} \cdot H^{(p)}\right)(l, .) .
$$

Portanto, como $V^{n_{p}}$ é um processo previsível, o processo $A^{n_{p}}$ é unicamente determinado pela função intensidade $h^{(p)}$. Nosso objetivo é estimar a função intensidade $h^{(p)}$ para determinarmos o modelo de intensidade multiplicativo. 
Um estimador para a função intensidade $h^{(p)}$ é um processo estocástico $\hat{h}^{n_{p}}:\{0,1,2, \cdots, k\} \rightarrow$ $[0,1]$ definido na base estocástica produto $\left(\left(S^{\infty}\right)^{n_{p}}, \mathcal{B}^{n_{p}},\left\{\mathcal{B}^{N^{n_{p}}}\right\}, P^{n_{p}}\right)$, no qual $P^{n_{p}}$ corresponde à probabilidade produto. Neste trabalho, um critério martingale é proposto para determinarmos um estimador $\hat{h}^{n_{p}}$ ótimo para a função intensidade. Desde que o martingale representa o ruído (parte não previsível), igualamos o martingale $Y^{n_{p}}$ a zero. Assim, para $i=1$

$$
\begin{aligned}
& Y^{n_{p}}(1, .)=N^{n_{p}}(1, .)-\hat{h}^{n_{p}}(1) \sum_{m=1}^{n_{p}} \mathbb{1}_{\left\{X_{m}^{(p)} \geq 1\right\}}=0 \\
& \Rightarrow \hat{h}^{n_{p}}(1)=\frac{N^{n_{p}}(1, .)}{\sum_{m=1}^{n_{p}} \mathbb{1}_{\left\{X_{m}^{(p)} \geq 1\right\}}}=\frac{N^{n_{p}}(1, .)}{V^{n_{p}}(1, .)} .
\end{aligned}
$$

Para $i=2$,

$$
\begin{aligned}
Y^{n_{p}}(2, .) & =N^{n_{p}}(2, .)-\left[\hat{h}^{n_{p}}(2) \sum_{m=1}^{n_{p}} \mathbb{1}_{\left\{X_{m}^{(p)} \geq 2\right\}}-\hat{h}^{n_{p}}(1) \sum_{m=1}^{n_{p}} \mathbb{1}_{\left\{X_{m}^{(p)} \geq 1\right\}}\right]=0 \\
Y^{n_{p}}(2, .) & =N^{n_{p}}(2, .)-\left[\hat{h}^{n_{p}}(2) V^{n_{p}}(2, .)-\hat{h}^{n_{p}}(1) V^{n_{p}}(1, .)\right]=0 \\
Y^{n_{p}}(2, .) & =N^{n_{p}}(2, .)-\left[\hat{h}^{n_{p}}(2) V^{n_{p}}(2, .)-\frac{N^{n_{p}}(1, .)}{V^{n_{p}}(1, .)} V^{n_{p}}(1, .)\right]=0 \\
\Rightarrow \hat{h}^{n_{p}}(2) & =\frac{\Delta N^{n_{p}}(2, .)}{V^{n_{p}}(2, .)}
\end{aligned}
$$

em que $\Delta N(2,)=.N(2,)-.N(1,$.$) . De forma geral, obtemos o estimador de Kaplan-Meier$

$$
\hat{h}^{n_{p}}(i)=\frac{\Delta N^{n_{p}}(i, .)}{V^{n_{p}}(i, .)},
$$

em que $\Delta N(i+1,)=.N(i+1,)-.N(i,$.$) , para i=1, \ldots, k$, com $\hat{h}^{n_{p}}(0)=0$. O estimador de $h^{(p)}$ está bem definido, pois podemos assumir, sem perda de generalidade, que $V^{n_{p}}(k,)>$.0 e, como $V^{n_{p}}(i,$.$) é um processo decrescente para i=1, \ldots, k, V^{n_{p}}(i,)>.0 \forall i$.

A partir daí, obtemos o estimador da distribuição de probabilidade da variável $W_{m}^{(p)}, m=$ $1, \ldots, n_{p}$,

$$
\begin{aligned}
\hat{\zeta}^{n_{p}}(i) & =\hat{h}^{n_{p}}(i) \prod_{l=1}^{i-1}\left[1-\hat{h}^{n_{p}}(l)\right] \\
& =\prod_{l=1}^{i}\left[1-\hat{h}^{n_{p}}(l)\right]^{V^{n_{p}}(l, .)-\Delta N^{n_{p}}(l, .)}\left[\hat{h}^{n_{p}}(l)\right]^{V^{n_{p}}(l, .)}
\end{aligned}
$$


para todo $i \in\{1, \ldots, k\}$ e também o estimador da função intensidade acumulada $H^{(p)}$

$$
\hat{H}^{n_{p}}(i)=\sum_{l=1}^{i} \hat{h}^{n_{p}}(l)=\sum_{l=1}^{i} \frac{\Delta N^{n_{p}}(l, .)}{V^{n_{p}}(l, .)}
$$

\subsubsection{Propriedades dos estimadores para amostras finitas}

Nesta seção estudadamos propriedades para amostras finitas dos estimadores $\hat{H}^{n_{p}}, \hat{h}^{n_{p}}$ e $\hat{\zeta}^{n_{p}}$. Inicialmente, observe que $\Delta N_{m}^{(p)}(i,$.$) pode assumir apenas dois valores, 0$ ou 1. A probabilidade dele assumir o valor 1 é

$$
P\left[\Delta N_{m}^{(p)}(i, .)=1\right]=P\left[X_{m}^{(p)}=1, X_{m}^{(p)}=W_{m}^{(p)}\right]=\zeta^{(p)}(i) .
$$

Logo, $\Delta N_{m}^{(p)}(i,$.$) tem distribuição de Bernoulli com parâmetro \zeta^{(p)}(i)$. Considerando que $\Delta N^{n_{p}}(i,)=.\sum_{m=1}^{n_{p}} \Delta N_{m}^{(p)}(i,$.$) , obtemos que$

$$
\Delta N^{n_{p}}(i, .) \sim \text { Binomial }\left(n_{p}, \zeta^{(p)}(i)\right), i=1, \ldots, k .
$$

Lema 3.1. O estimador $\hat{H}^{n_{p}}$ da função intensidade acumulada $H^{(p)}$ pode ser escrito como

$$
\hat{H}^{n_{p}}(i)=\left(\frac{1}{V^{n_{p}}} \cdot Y^{n_{p}}\right)(i, .)+H^{(p)}(i) .
$$

Demonstração. Como apresentado na equação (3.15), o estimador da função de intensidade acumulada é

$$
\hat{H}^{n_{p}}(i)=\sum_{l=1}^{i} \hat{h}^{n_{p}}(l)=\sum_{l=1}^{i} \frac{\Delta N^{n_{p}}(l, .)}{V^{n_{p}}(l, .)}
$$

Pela Decomposição de Doob-Meyer, obtemos

$$
\begin{aligned}
\sum_{l=1}^{i} \frac{\Delta N^{n_{p}}(l, .)}{V^{n_{p}}(l, .)} & =\sum_{l=1}^{i} \frac{\Delta\left[Y^{n_{p}}(l, .)+A^{n_{p}}(l, .)\right]}{V^{n_{p}}(l, .)} \\
& =\sum_{l=1}^{i} \frac{\Delta Y^{n_{p}}(l, .)}{V^{n_{p}}(l, .)}+\sum_{l=1}^{i} \frac{\Delta A^{n_{p}}(l, .)}{V^{n_{p}}(l, .)} \\
& =\left(\frac{1}{V^{n_{p}}} \cdot Y^{n_{p}}\right)(i, .)+\sum_{l=1}^{i} h^{(p)}(l) \\
& =\left(\frac{1}{V^{n_{p}}} \cdot Y^{n_{p}}\right)(i, .)+H^{(p)}(i) .
\end{aligned}
$$


Com esses resultados, conseguimos provar os próximos teoremas.

Teorema 3.2. Considere o estimador $\hat{H}^{n_{p}}$ da função intensidade acumualada $H^{(p)}$ e o estimador $\hat{h}^{n_{p}}$ da função intensidade $h^{(p)}$. Temos que

$$
E\left[\hat{H}^{n_{p}}(i)\right]=H^{(p)}(i)
$$

$e$

$$
E\left[\hat{h}^{n_{p}}(i)\right]=h^{(p)}(i)
$$

para $i=1, \ldots, k$.

Demonstração. (a) Por definição, $V^{n_{p}}(i,),. i=1, \ldots, k$ é um processo previsível, $\operatorname{logo} \frac{1}{V^{n_{p}}(i, .)}$ também é um processo previsível. Assim, pelo Teorema 2.2 obtemos que $\left(\frac{1}{V^{n} p} . Y^{n_{p}}\right)(i,$.$) ,$ $i=1, \ldots, k$ é um martingale, o que implica que $\mathrm{E}\left[\left(\frac{1}{V^{n p}} . Y^{n_{p}}\right)(i,).\right]=0, i=1, \ldots, k$. Com isso, obtemos do Lema 3.1 que

$$
\mathrm{E}\left[\hat{H}^{n_{p}}(i, .)\right]=H^{(p)}(i, .), i \leq k
$$

ou seja, o estimador $\hat{H}^{n_{p}}$ é um estimador não-viciado da função $h^{(p)}$

(b) Pelas equações (3.12) e (3.15) temos que

$$
\hat{H}^{n_{p}}(i)-H^{(p)}(i)=\sum_{l=1}^{i} \hat{h}^{n_{p}}(l)-\sum_{l=1}^{i} h^{(p)}(l)=\sum_{l=1}^{i}\left[\hat{h}^{n_{p}}(l)-h^{(p)}(l)\right] .
$$

Pelo item (a) $\mathrm{E}\left[\hat{H}^{n_{p}}(1)\right]=H^{(p)}(1)$, o que implica que $\mathrm{E}\left[\hat{h}^{n_{p}}(1)\right]=h^{(p)}(1)$. Da mesma forma, $\mathrm{E}\left[\hat{H}^{n_{p}}(2)\right]=H^{(p)}(2)$ e, portanto,

$$
\mathrm{E}\left[\sum_{l=1}^{2} \hat{h}^{n_{p}}(l)\right]=\sum_{l=1}^{2} h^{(p)}(l) \Rightarrow \sum_{l=1}^{2} \mathrm{E}\left[\hat{h}^{n_{p}}(l)\right]=\sum_{l=1}^{2} h^{(p)}(l) \Rightarrow \mathrm{E}\left[\hat{h}^{n_{p}}(2)\right]=\sum_{l=1}^{2} h^{(p)}(l)
$$

Assumamos que vale para $k-1$, ou seja,

$$
\mathrm{E}\left[\hat{H}^{n_{p}}(k-1)\right]=H^{(p)}(k-1) .
$$

Provando por indução sobre $k$, temos que $\mathrm{E}\left[\hat{H}^{n_{p}}(k)\right]=H^{(p)}(k)$, o que implica que

$$
\mathrm{E}\left[\sum_{l=1}^{k} \hat{h}^{n_{p}}(l)\right]=\sum_{l=1}^{k} h^{(p)}(l) \Rightarrow \mathrm{E}\left[\sum_{l=1}^{k-1} \hat{h}^{n_{p}}(l)\right]+\mathrm{E}\left[\hat{h}^{n_{p}}(k)\right]=\sum_{l=1}^{k-1} h^{(p)}(l)+h^{(p)}(k)
$$


e, portanto,

$$
E\left[\hat{h}^{n_{p}}(k)\right]=h^{(p)}(k)
$$

Assim, conluímos que

$$
E\left[\hat{h}^{n_{p}}(l)\right]=h^{(p)}(l), l=1, \ldots, k
$$

ou seja, o estimador $\hat{h}^{n_{p}}$ é um estimador não-viciado para a função $h^{(p)}$.

Para dados censurados, $\zeta^{\hat{n}_{p}}$ é um estimador viciado para $\zeta^{(p)}$. Porém, se tivermos dados não censurados, obtemos

$$
\begin{aligned}
\hat{\zeta}^{n_{p}}(i) & =\hat{h}^{n_{p}}(i) \prod_{l=1}^{i-1}\left[1-\hat{h}^{n_{p}}(l)\right] \\
& =\prod_{l=1}^{i}\left[1-\hat{h}^{n_{p}}(l)\right]^{V^{n_{p}}(l, .)-\Delta N^{n_{p}}(l, .)}\left[\hat{h}^{n_{p}}(l)\right]^{V^{n_{p}}(l, .)} \\
& =\frac{\Delta N^{\left(n_{p}\right)(i, .)}}{n_{p}}
\end{aligned}
$$

que corresponde ao estimador empírico da probabilidade $\zeta^{(p)}$. Para este caso, podemos mostrar que o estimador é não-viciado.

Teorema 3.3. Para dados não censurados, o estimador $\hat{\zeta}^{\hat{n}_{p}}$ da função de distribuição de probabilidade $\zeta^{(p)}$ é um estimador não-viciado.

Demonstração. Como visto anteriormente, $\Delta N^{n_{p}}(l,.) \sim$ Binomial $\left(n_{p}, \zeta^{(p)}(l)\right)$. Consequentemente, temos que $E\left[\Delta N^{n_{p}}(l,).\right]=n_{p} \zeta_{l}^{(p)}$. Utilizando esta informação e a equação 3.19 , temos que

$$
\mathrm{E}\left[\hat{\zeta}^{n_{p}}(l)\right]=\mathrm{E}\left[\frac{\Delta N^{n_{p}}(l, .)}{n_{p}}\right]=\zeta^{(p)}(l) .
$$

Portanto, o estimador $\zeta^{\hat{n}_{p}}$ da função de distribuição de probabilidade $\zeta^{(p)}$ é um estimador não-viciado.

Na seqüência são determinadas as variâncias dos estimadores $\hat{h}^{n_{p}}$ e $\hat{H}^{n_{p}}$. Para determinarmos a variância do estimador $\hat{H}^{n_{p}}$ utilizamos o processo variação quadrática associado ao martingale $\left(\frac{1}{V^{n_{p}}} \cdot Y^{n_{p}}\right)$. 
Teorema 3.4. As variâncias dos estimadores $\hat{H}^{n_{p}}$ e $\hat{h}^{n_{p}}$ são dadas, respectivamente, por

$$
\operatorname{Var}\left[\hat{H}^{n_{p}}(i)\right]=\sum_{l=1}^{i}\left[h^{(p)}(l)-h^{(p)^{2}}(l)\right] E\left[\frac{1}{V^{n_{p}}(l, .)}\right], i \in\{1, \ldots, k\}
$$

e

$$
\operatorname{Var}\left[\hat{h}^{n_{p}}(l)\right]=\left[h^{(p)}(l)-h^{(p)^{2}}(l)\right] E\left[\frac{1}{V^{n_{p}(l, .)}}\right], l \leq i .
$$

Demonstração. Utilizando o Lema 3.1, podemos escrever a diferença entre o estimador $\hat{H}^{n_{p}}(i)$ e a variável aleatória $H^{(p)}(i)$ como

$$
\hat{H}^{n_{p}}(i, .)-H^{(p)}(i, .)=\left(\frac{J^{n_{p}}}{V^{n_{p}}} \cdot Y^{n_{p}}\right)(i, .)
$$

Utilizando esta igualdade, a definição de processo variação quadrática e a Proposição 2.3, obtemos que

$$
\begin{aligned}
E\left[\hat{H}^{n_{p}}(i, .)-H^{(p)}(i, .)\right]^{2} & =E\left[\left(\frac{1}{V^{n_{p}}} \cdot Y^{n_{p}}\right)(i, .)\right]^{2}=E\left[<\left(\frac{1}{V^{n_{p}}} \cdot Y^{n_{p}}\right) ;\left(\frac{1}{V^{n_{p}}} \cdot Y^{n_{p}}\right)>\right] \\
& =E\left[\left(\left(\frac{1}{V^{n_{p}}}\right)^{2} \cdot<Y^{n_{p}}, Y^{n_{p}}>\right)(i, .)\right]
\end{aligned}
$$

No entanto, temos que

$$
\begin{aligned}
\left(\left(\frac{1}{V^{n_{p}}}\right)^{2} \cdot<Y^{n_{p}}, Y^{n_{p}}>\right)(i, .)= & \\
& =\sum_{l=1}^{i}\left(\frac{1}{V^{n_{p}}(l, .)}\right)^{2} \Delta<Y^{n_{p}}, Y^{n_{p}}>(l, .)=\sum_{l=1}^{i}\left(\frac{1}{V^{n_{p}}(l, .)}\right)^{2} \Delta \sum_{m=1}^{n_{p}}<Y_{m}^{(p)}, Y_{m}^{(p)}>(l, .) \\
& =\sum_{l=1}^{i}\left(\frac{1}{V^{n_{p}}(l, .)}\right)^{2} \sum_{m=1}^{n_{p}}\left[\Delta A_{m}^{(p)}(l, .)-\Delta A_{m}^{(p)^{2}}(l, .)\right] \\
& =\sum_{l=1}^{i} \frac{1}{V^{n_{p}}(l, .)} \sum_{m=1}^{n_{p}}\left[h^{(p)}(l) V_{m}^{(p)}(l, .)-h^{(p)^{2}}(l) V_{m}^{(p)}{ }^{2}(l, .)\right] \\
& =\sum_{l=1}^{i} \frac{1}{V^{n_{p}}(l, .)}\left[h^{(p)}(l) V^{n_{p}}(l, .)-h^{(p)^{2}}(l) V^{n_{p}}(l, .)\right. \\
& =\sum_{l=1}^{i} \frac{1}{V^{n_{p}(l, .)}}\left[h^{(p)}(l)-h^{(p)^{2}}(l)\right]
\end{aligned}
$$


Assim, a variância do estimador $\hat{H}^{n_{p}}$ é dado por

$$
\operatorname{Var}\left[\hat{H}^{n_{p}}(i)\right]=\sum_{l=1}^{i}\left[h^{(p)}(l)-h^{(p)^{2}}(l)\right] \mathrm{E}\left[\frac{1}{V^{n_{p}}(l, .)}\right] .
$$

Para obtermos a variância do estimador $\hat{h}^{n_{p}}$ consideremos o processo $S^{n_{p}}(l)$ tal que $S^{n_{p}}(l)=$ $\hat{H}^{n_{p}}(l)-H^{(p)}(l)$. Observe que $S^{n_{p}}(l)$ é um martingale, $l \leq i$. Além disso, temos que

$$
E\left[\hat{h}^{n_{p}}(l)-h^{(p)}(l)\right]^{2}=E\left[S^{n_{p}}(l)-S^{n_{p}}(l-1)\right]^{2} .
$$

Por outro lado, temos que

$$
\begin{aligned}
E\left[S^{n_{p}}(l)-S^{n_{p}}(l-1)\right]^{2} & =E\left[\left(\frac{1}{V^{n_{p}}} \cdot Y^{n_{p}}\right)(l, .)-\left(\frac{1}{V^{n_{p}}} \cdot Y^{n_{p}}\right)(l-1, .)\right] \\
& =\left[h^{(p)}(l)-h^{(p)^{2}}(l)\right] \mathrm{E}\left[\frac{1}{V^{n_{p}}(l, .)}\right] .
\end{aligned}
$$

Portanto,

$$
\operatorname{Var}\left[\hat{h}^{n_{p}}(l)\right]=\left[h^{(p)}(l)-h^{(p)^{2}}(l)\right] \mathrm{E}\left[\frac{1}{V^{n_{p}}(l, .)}\right] .
$$

Proposição 3.1. Consideremos as variáveis aleatórias $V_{m}^{(p)}(l,)=.\mathbb{1}_{\left\{X_{m}^{(p)} \geq l\right\}}$ e $V^{n_{p}}(l,)=$. $\sum_{m=1}^{n_{p}} V_{m}^{(p)}(l,$.$) . Denotamos por$

$$
\theta^{(p)}(l)=\eta^{(p)}(l)+\eta^{(p)}(l+1)+\cdots+\eta^{(p)}(k+1)
$$

$\theta^{(p)}(l)>0, \forall l, p$.

(a) A variável aleatória $V_{m}^{(p)}(l,$.$) tem distribuição de Bernoulli com parâmetro \theta^{(p)}(l)$.

(b) A variável aleatória $V^{n_{p}}(l,$.$) tem distribuinção Binomial com parâmetros n_{p}$ e $\theta^{(p)}(l)$.

Demonstração. (a) A variável aleatória $V_{m}^{(p)}$ pode assumir apenas dois valores, 0 ou 1 . A probabilidade dele assumir o valor 1 é

$$
\begin{aligned}
P\left[V_{m}^{(p)}(l, .)=1\right] & =P\left[X_{m}^{(p)} \geq l\right] \\
& =1-\left(\eta^{(p)}(1)+\eta^{(p)}(2)+\cdots+\eta^{(p)}(l-1)\right)>0 \\
& =\theta^{n_{p}}(l)
\end{aligned}
$$

ou seja, o processo $V_{m}^{(p)}(l,$.$) tem distribuição de Bernoulli com parâmetro \theta_{l}^{(p)}$. 
(b) Como $V^{n_{p}}(l,)=.\sum_{m=1}^{n_{p}} V_{m}^{(p)}(l,$.$) obtemos do item anterior que$

$$
V^{n_{p}}(l, .) \sim \operatorname{Binomial}\left(n_{p}, \theta^{(p)}(l)\right)
$$

Utilizando este resultado, será estudada a distribuição exata do estimador $\hat{h}^{n_{p}}$. Como a distribuição condicional de $\Delta N^{n_{p}}(i,.) / V^{n_{p}}(i,$.$) é uma Binomial com parâmetros V^{n_{p}}(i,$.$) e$ $h^{(p)}(i)$, concluímos que para todo $0<a \leq b \leq n$,

$$
\begin{aligned}
P\left[\Delta N^{\left(n_{p}\right)}(i, .)=a ; V^{n_{p}}(i, .)=b\right] & =P\left[\Delta N^{n_{p}}(i, .)=a / V^{n_{p}}(i, .)=b\right] P\left[V^{n_{p}}(i, .)=b\right] \\
& =\left(\begin{array}{c}
b \\
a
\end{array}\right)\left(h^{(p)}(i)\right)^{a}\left(1-h^{(p)}(i)\right)^{b-a}\left(\begin{array}{c}
n \\
b
\end{array}\right)\left(\theta^{(p)}(i)\right)^{b}\left[1-\theta^{(p)}(i)\right]^{n-b}
\end{aligned}
$$

Com isso, a distribuição exata do estimador $\hat{h}^{n_{p}}(i)$ é obtida por

$$
P\left[\hat{h}^{n_{p}}=\frac{\Delta N^{n_{p}}(i, .)}{V^{n_{p}}(i, .)}=\frac{a}{b}\right]=P\left[\Delta N^{n_{p}}(i, .)=a ; V^{n_{p}}(i, .)\right] .
$$

\subsubsection{Propriedades assintóticas}

Nesta seção, vamos estudar algumas propriedades assintóticas relacionadas ao estimador $\hat{H}^{n_{p}}$ do processo estocástico $H^{n_{p}}$ e, conseqüetemente, do estimador de Kaplan-Meier. Primeiramente, vamos verificar alguns resultados necessários para o estudo das propriedades assintóticas dos estimadores. Depois, iremos mostrar a consistência do estimador.

Teorema 3.5. Dada uma seqüência $\hat{H}^{n_{p}}$ de estimadores da função intensidade $H^{(p)}$ temos que

$$
P\left[\sup _{l \leq i}\left|\hat{H}^{n_{p}}(l)-H^{(p)}(l)\right|\right] \rightarrow 0
$$

ou seja, $\hat{H}^{n_{p}}$ é um estimador consistente para a função $H^{(p)}$.

Demonstração. Pela desigualdade de Doob (Teorema 1, pp. 464 de Shiryayev (1984)) e desigualdade de Burkholder (Shiryayev (1984), pp. 469) obtemos que

$$
P\left[\sup _{l \leq i}\left|\hat{H}^{n_{p}}(l)-H^{(p)}(l)\right|\right] \leq \frac{c}{\lambda^{2}} \sum_{l=1}^{i}\left[h^{(p)}(l)-h^{(p)^{2}}(l)\right] \mathrm{E}\left[\frac{1}{V^{n_{p}(l, .)}}\right]
$$


Por outro lado, pela Proposição 3.1 temos que $V_{m}^{(p)}(l,.) \sim$ Bernoulli $\left(\theta^{n_{p}}(l)\right)$. Definamos o conjunto $S^{n_{p}}=\left\{V_{m}^{(p)}(l,)=1.\right\}$. Como $P\left[S^{n_{p}}\right]=\theta_{l}^{n_{p}}$,

$$
\sum_{n_{p}=1}^{\infty} P\left[S^{n_{p}}\right]=+\infty
$$

para todo $p=1, \ldots J$. Além disso, $S^{n_{1}}, \cdots, S^{n_{p}}$ são independentes. Logo, pelo Lema de Borel-Cantelli, temos que

$$
P\left[\lim \sup _{n_{p}} S^{n_{p}}\right]=1
$$

ou seja, a probabilidade de ocorrer $S^{n_{p}}$ é 1, exceto num número finito de pontos. Portanto,

$$
V^{n_{p}}(l, .)=\sum_{m=1}^{n_{p}} V_{m}^{(p)}(l, .) \rightarrow+\infty, \quad P-q . c ., \quad \text { quando } n_{p} \rightarrow \infty .
$$

Conseqüentemente, $\frac{1}{V^{n_{p}}(l, .)}$ converge quase certamente para zero. Portanto, pelo Teorema da Convergência Monótona, $E\left[\frac{1}{V^{n_{p}}(l, .)}\right] \rightarrow 0$. Assim, temos que $\hat{H}^{n_{p}}$ é um estimador consistente para a função intensidade acumulada $H^{(p)}$.

Teorema 3.6. Dada uma seqüência $\hat{h}^{n_{p}}$ de estimadores da função intensidade $h^{(p)}$ temos que

$$
P\left[\left|\hat{h}^{n_{p}}(l)-h^{(p)}(l)\right|\right] \rightarrow 0,
$$

ou seja, $\hat{h}^{n_{p}}$ é um estimador consistente para a função $h^{(p)}$.

Demonstração. Consideremos $l=1$. Neste caso, temos que

$$
\left|\hat{H}^{n_{p}}(1)-H^{(p)}(1)\right|=\left|\hat{h}^{n_{p}}(1)-h^{(p)}(1)\right|
$$

e portanto, pelo Teorema 3.5, concluímos que

$$
P\left[\left|\hat{h}^{n_{p}}(1)-h^{(p)}(1)\right|\right] \rightarrow 0 .
$$

Por outro lado temos que

$$
\begin{aligned}
\hat{H}^{n_{p}}(i)-H^{(p)}(i) & =\sum_{l=1}^{i} \hat{h}^{n_{p}}(l)-\sum_{l=1}^{i} h^{(p)}(l) \\
& =\sum_{l=1}^{i-1}\left[\hat{h}^{n_{p}}(l)-h^{(p)}(l)\right]+\left[\hat{h}^{n_{p}}(i)-h^{(p)}(i)\right]
\end{aligned}
$$




$$
\Rightarrow\left[\hat{h}^{n_{p}}(i)-h^{(p)}(i)\right]=\left[\hat{H}^{n_{p}}(i)-H^{(p)}(i)\right]-\sum_{l=1}^{i-1}\left[\hat{h}^{n_{p}}(l)-h^{(p)}(l)\right]
$$

Portanto,

$$
\left|\hat{h}^{n_{p}}(i)-h^{(p)}(i)\right| \leq\left|\hat{H}^{n_{p}}(i)-H^{(p)}(i)\right|+\sum_{l=1}^{i-1}\left|\hat{h}^{n_{p}}(l)-h^{(p)}(l)\right|
$$

Assumindo que vale para $i-1$ e utilizando o Teorema 3.5, concluímos por indução que vale para $i$. Portanto,

$$
P\left[\left|\hat{h}^{n_{p}}(l)-h^{(p)}(l)\right|\right] \rightarrow 0, l=1, \ldots, i
$$

Teorema 3.7. O estimador $\hat{\zeta}^{n_{p}}$ é um estimador consistente para a função $\zeta^{(p)}$.

Demonstração. Pela equação 3.14 temos que o estimador $\hat{\zeta}^{n_{p}}$ é função contínua do estimador da função intensidade, $\hat{h}^{n_{p}}$. Como $\hat{h}^{n_{p}}$ é estimador consistente para $h^{(p)}$, concluímos que $\hat{\zeta}^{n_{p}}$ é estimador consistente para $\zeta^{(p)}$. 


\section{Capítulo 4}

\section{Distribuição Assintótica de Integrais \\ Estocásticas}

Nesta seção desenvolvemos um teorema do limite central para as integrais estocásticas associ-

adas ao processo de contagem $N^{n_{p}}$. Essa teoria foi baseada nos resultados de Helland (1982). Esses resultados são importantes para que possamos desenvolver os testes de hipótese.

Para que possamos desenvolver o teorema do limite central, precisamos definir uma filtragem em relação ao número de itens (ou indivíduos), pois o limite é tomado neste número.

\subsection{Amostra Única}

Consideremos uma amostra aleatória simples

$$
\left(\left(W_{1}^{(1)}, C_{1}^{(1)}\right), \ldots,\left(W_{n_{1}}^{(1)}, C_{n_{1}}^{(1)}\right)\right)
$$

com as mesmas características da amostra 3.7 definida na Seção 3.3. Para $n_{1} \in \mathbb{N}$ e $l \in\{1, \ldots, i\}$ fixos, com $i \in\{1, \ldots, k\}$ e $k \in \mathbb{N}$, definimos a álgebra

$$
\vartheta_{l}(0)=\text { álgebra gerada por }\left\{V^{n_{1}}(l, .) ; V_{1}^{(1)}(l, .)\right\},
$$

$\vartheta_{l}(q)=$ álgebra gerada $\operatorname{por}\left\{V^{n_{1}}(l,.) ; V_{1}^{(1)}(l,),. \ldots, V_{q+1}^{(1)}(l,.) ; \Delta N_{1}^{(1)}(l,),. \ldots, \Delta N_{q}^{(1)}(l,.) ; C_{1}^{(1)}, \ldots, C_{n_{1}}^{(1)}\right\}$ para $q \in\left\{1, \ldots, n_{1}-1\right\}$ fixo e

$\vartheta_{l}\left(n_{1}\right)=$ álgebra gerada $\operatorname{por}\left\{V^{n_{1}}(l,.) ; V_{1}^{(1)}(l,),. \ldots, V_{n_{1}}^{(1)}(l,.) ; \Delta N_{1}^{(1)}(l,),. \ldots, \Delta N_{n_{1}}^{(1)}(l,.) ; C_{1}^{(1)}, \ldots, C_{n_{1}}^{(1)}\right\}$ 
Definimos a álgebra $\gamma_{i}(j)$, para $0 \leq j \leq n_{1}$, como sendo o acumulado da álgebra $\vartheta_{l}(j)$ até a categoria $i$. Assim,

$$
\begin{aligned}
& \gamma_{i}(0)=\text { álgebra gerada por }\left\{\vartheta_{1}(0), \vartheta_{2}(0), \ldots, \vartheta_{i}(0)\right\} \\
& \gamma_{i}(q)=\text { álgebra gerada por }\left\{\vartheta_{1}(q), \vartheta_{2}(q), \ldots, \vartheta_{i}(q)\right\}
\end{aligned}
$$

para $q=1, \ldots, n_{1}-1$ fixo e

$$
\gamma_{i}\left(n_{1}\right)=\text { álgebra gerada por }\left\{\vartheta_{1}\left(n_{1}\right), \vartheta_{2}\left(n_{1}\right), \ldots, \vartheta_{i}\left(n_{1}\right)\right\}
$$

Consideremos $\left\{\gamma_{i}\right\}$ a filtragem formada pelas álgebras encaixantes $\gamma_{i}(j), 0 \leq j \leq n_{1}$. Seja $U^{n_{1}}(l,$.$) uma variável aleatória com respeito à álgebra \gamma_{i}(0)$ para $l=1, \ldots i$ fixo, de tal forma que as seguintes propriedades são válidas

(a) Para $n_{1}$ fixo o processo $U^{n_{1}}$ é previsível na filtragem $\left\{\mathcal{B}^{N^{n_{1}}}\right\}$.

(b) $\mathrm{E}\left[\left|U^{n_{1}}(l,).\right|\right] \rightarrow 0$ quando $n_{1} \rightarrow \infty, \forall l=1, \ldots, i$

(c) $\mathrm{E}\left[U^{n_{1} 2}(l,.) V^{n_{1}}(l,).\right] \rightarrow G_{l}$ quando $n_{1} \rightarrow \infty$, para algum $G_{l}>0$ e $\forall l=1, \ldots, i$.

Seja $a_{m}=\sum_{l=1}^{i} U^{n_{1}}(l,.) \Delta Y_{m}^{(1)}(l,$.$) , para 0 \leq m \leq n_{1}$. Definimos a somas parciais $R_{j}(i)=$ $\sum_{m=1}^{j} a_{m}$, para $0 \leq j \leq n_{1}$ e $i \in\{1, \ldots, k\}$ fixo, com $R_{0}(i)=0$. Definimos o processo estocástico

$$
R(i)=\left\{R_{0}(i), R_{1}(i), \ldots, R_{n_{1}}(i)\right\}
$$

que é adaptado à fitragem $\left\{\gamma_{i}\right\}$ por construção.

Proposição 4.1. O processo estocástico $R(i)$, para $i \in\{1, \ldots, k\}$ fixo, é um martingale com respeito à filtragem $\left\{\gamma_{i}\right\}$.

Demonstração. O processo $R(i)$ é adaptado à filtragem $\left\{\gamma_{i}\right\}$. Para demonstrar que esse processo é um martingale, basta provar que $\mathrm{E}\left[R_{j}(i) \mid \gamma_{i}(j-1)\right]=R_{j-1}(i)$, conforme a Definição 2.3, que é o mesmo que mostrar que $\mathrm{E}\left[R_{j}(i)-R_{j-1} \mid \gamma_{i}(j-1)\right]=0$. Mas observe que

$$
R_{j}(i)-R_{j-1}(i)=\sum_{l=1}^{i} U^{n_{1}}(l, .) \Delta Y_{j}^{(1)}(l, .)=a_{j}(i)
$$


Portanto, basta mostrar que

$$
E\left[a_{j}(i) \mid \gamma_{i}(j-1)\right]=0
$$

para $i \in\{1, \ldots, k\}$ fixo e $j=1, \ldots, n_{1}$. Inicialmente, temos que

$$
\begin{aligned}
E\left[\sum_{l=1}^{i} U^{n_{1}}(l, .)\right. & \left.\Delta Y_{j}^{(1)}(l, .) \mid \gamma_{i}(j-1)\right] \\
& =\sum_{l=1}^{i} U^{n_{1}}(l, .) \mathrm{E}\left[\Delta N_{j}^{(1)}(l .)-\Delta A_{j}^{(1)}(l .) \mid \gamma_{i}(j-1)\right] \\
& =\sum_{l=1}^{i} U^{n_{1}}(l, .)\left\{\mathrm{E}\left[\Delta N_{j}^{(1)}(l .) \mid \gamma_{i}(j-1)\right]+\mathrm{E}\left[\Delta A_{j}^{(1)}(l, .) \mid \gamma_{i}(j-1)\right]\right\}
\end{aligned}
$$

Observe que $\Delta A_{j}^{(1)}(l,$.$) é mensurável em \gamma_{i}(j-1)$ para $j=1, \ldots, n_{1}$ e $l=1, \ldots, i$. Além disso, temos ques $\Delta N_{j}^{(1)}(l,$.$) condicionado a V_{j}^{(1)}(l,$.$) é independente de todos os outros termos da$ filtragem, $\gamma_{i}(j-1)$, também para $j=1, \ldots, n_{1}$ e $l=1, \ldots, i$. Com isso,

$$
\begin{aligned}
E\left[\sum_{l=1}^{i} U^{n_{1}}(l, .) \Delta Y_{j}^{(1)}\right. & \left.(l, .) \mid \gamma_{i}(j-1)\right] \\
& =\sum_{l=1}^{i} U^{n_{1}}(l, .)\left\{\mathrm{E}\left[\Delta N_{j}^{(1)}(l .) \mid V_{j}^{(1)}(l, .)\right]-\Delta A_{j}^{(1)}(l, .)\right\} \\
& =\sum_{l=1}^{i} U^{n_{1}}(l, .)\left\{\mathrm{E}\left[\Delta N_{j}^{(1)}(l .) \mid V_{j}^{(1)}(l, .)\right]-V_{j}^{(1)}(l, .) h^{(1)}(l)\right\} \\
& =0
\end{aligned}
$$

pois $\Delta N_{j}^{(1)}(l,) \mid. V_{j}^{(1)}(l,$.$) tem distribuição binomial com número de ensaios V_{j}^{(1)}(l,$.$) e probabil-$ idade $h^{(1)}(l)$, para $j=1, \ldots, n_{1}$ e $l=1, \ldots, i$. Assim, concluímos que $R(i)$ é um martingale em relação a filtragem $\left\{\gamma_{i}\right\}$.

Da Proposição 2.2, concluímos que o processo $\left\{a_{j}(i), 0 \leq j \leq n_{1}\right\}$ é um martingale array difference em relaçao a filtragem $\left\{\gamma_{i}\right\}$.

Lema 4.1. A seguinte propriedade é válida para os termos do processo $R(i)$

$$
E\left[\max _{1 \leq j \leq n_{1}}\left|a_{j}(i)\right|\right] \rightarrow 0, \quad \text { quando } n_{1} \rightarrow \infty .
$$


Demonstração. Por propriedades de máximo e módulo temos que

$$
\begin{aligned}
\max _{1 \leq j \leq n_{1}}\left|a_{j}(i)\right|=\max _{1 \leq j \leq n_{1}}\left|\sum_{l=1}^{i} U^{n_{1}}(l, .) \Delta Y_{j}^{(1)}(l .)\right| & \leq \max _{1 \leq j \leq n_{1}} \sum_{l=1}^{i}\left|U^{n_{1}}(l, .) \Delta Y_{j}^{(1)}(l .)\right| \\
& \leq \sum_{l=1}^{i} \max _{1 \leq j \leq n_{1}}\left|U^{n_{1}}(l, .) \Delta Y_{j}^{(1)}(l .)\right|
\end{aligned}
$$

Com isso,

$$
\mathrm{E}\left[\max _{1 \leq j \leq n_{1}}\left|\sum_{l=1}^{i} U^{n_{1}}(l, .) \Delta Y_{j}^{(1)}(l .)\right|\right] \leq \mathrm{E}\left[\sum_{l=1}^{i} \max _{1 \leq j \leq n_{1}}\left|U^{n_{1}}(l, .) \Delta Y_{j}^{(1)}(l .)\right|\right] .
$$

Por outro lado, como $\Delta N_{j}^{(1)}(l,) \leq$.1 e $A_{j}^{(1)}(l,) \leq$.1 , temos que

$$
\begin{aligned}
\left|U^{n_{1}}(l, .) \Delta Y_{j}^{(1)}(l .)\right| & =\left|U^{n_{1}}(l, .)\left[\Delta N_{j}^{(1)}(l, .)-\Delta A_{j}^{(1)}(l, .)\right]\right| \\
& \leq\left|U^{n_{1}}(l, .) \Delta N_{j}^{(1)}(l, .)\right|+\left|U^{n_{1}}(l, .) \Delta A_{j}^{(1)}(l, .)\right| \\
& \leq\left|U^{n_{1}}(l, .)\right|+\left|U^{n_{1}}(l, .)\right|=2\left|U^{n_{1}}(l, .)\right|
\end{aligned}
$$

Das equações 4.4 e 4.5, obtemos que

$$
\mathrm{E}\left[\max _{1 \leq j \leq n_{1}}\left|\sum_{l=1}^{i} U^{n_{1}}(l, .) \Delta Y_{j}^{(1)}(l .)\right|\right] \leq \mathrm{E}\left[\max _{1 \leq j \leq n_{1}} 2 i U^{n_{1}}(l, .)\right]=2 i \mathrm{E}\left[U^{n_{1}}(l, .)\right]
$$

para $i \in\{1, \ldots, k\}$ fixo, com $k \in \mathbb{N}$. Por hipóteste $E\left[\left|U^{n_{1}}(l,).\right|\right] \rightarrow 0$ quando $n_{1} \rightarrow \infty, \forall l$. Portanto,

$$
\mathrm{E}\left[\max _{1 \leq j \leq n_{1}}\left|\sum_{l=1}^{i} U^{n_{1}}(l, .) \Delta Y_{j}^{(1)}(l .)\right|\right] \rightarrow 0, \text { quando } n_{1} \rightarrow \infty
$$

Lema 4.2. Seja $\sigma^{2}(i)=\sum_{l=1}^{i}\left[h^{(1)}(l)-h^{(1)^{2}}(l)\right] G_{l}$. Então,

$$
\sum_{j=1}^{n_{1}}\left[a_{j}(i)\right]^{2} \rightarrow \sigma^{2}(i)
$$

Demonstração. Pela preposição 4.1, o processo $R(i)$ é um martingale e, portanto, pela Proposição 2.1 temos que

$$
\mathrm{E}\left[R_{j}^{2}(i)\right]-\mathrm{E}\left[R_{0}^{2}(i)\right]=\sum_{j=0}^{n_{1}-1} \mathrm{E}\left[\left(\Delta R_{j+1}(i)\right)^{2}\right]
$$


Os termos do processo $R(i)$ podem ser escritos da seguinte forma $R_{j}(i)=\sum_{m=1}^{j} \Delta R_{m}(i)$. Assim, podemos reescrever a equação 4.8

$$
\mathrm{E}\left[\left(\sum_{j=1}^{n_{1}} \Delta R_{j}(i)\right)^{2}\right]=\sum_{j=0}^{n_{1}-1} \mathrm{E}\left[\left(\Delta R_{j+1}(i)\right)^{2}\right]=\sum_{j=1}^{n_{1}} \mathrm{E}\left[\left(\Delta R_{j}(i)\right)^{2}\right]
$$

Por outro lado, observe que $\Delta R_{j}(i)=a_{j}(i)$, para todo $0 \leq j \leq n_{1}$, e, como $a_{j}(i)=\sum_{l=1}^{i} U^{n_{1}}(l,.) \Delta Y_{j}^{(1)}(l,$.$) ,$ obtemos que

$$
\begin{aligned}
\mathrm{E}\left[\sum_{j=1}^{n_{1}}\left[a_{j}(i)\right]^{2}\right] & =\mathrm{E}\left[\left(\sum_{j=1}^{n_{1}} a_{j}(i)\right)^{2}\right] \\
& =E\left[\left(\sum_{j=1}^{n_{1}} \sum_{l=1}^{i} U^{n_{1}}(l, .) \Delta Y_{j}^{(1)}(l, .)\right)^{2}\right] \\
& =E\left[\left(\sum_{l=1}^{i} U^{n_{1}}(l, .) \Delta Y^{n_{1}}(l, .)\right)^{2}\right]
\end{aligned}
$$

Utilizando a definição de integral estocástica e a Proposição 2.3, temos que

$$
\begin{aligned}
E\left[\left(\sum_{l=1}^{i} U^{n_{1}}(l, .) \Delta Y^{n_{1}}(l, .)\right)^{2}\right] & =E\left[\left(U^{n_{1}} \cdot Y^{n_{1}}\right)^{2}(i, .)\right] \\
& =E\left[<\left(U^{n_{1}} \cdot Y^{n_{1}}\right) ;\left(U^{n_{1}} \cdot Y^{n_{1}}\right)>(i, .)\right] \\
& =E\left[\left(U^{n_{1}} \cdot<Y^{n_{1}}, Y^{n_{1}}>\right)(i, .)\right]
\end{aligned}
$$


Utilizando a equação 2.9,

$$
\begin{aligned}
\left(U^{n_{1} 2} .<Y^{n_{1}}, Y^{n_{1}}>\right)(i, .) & =\sum_{l=1}^{i} U^{n_{1} 2}(l, .) \Delta<Y^{n_{1}}, Y^{n_{1}}>(l, .) \\
& =\sum_{l=1}^{i} U^{n_{1} 2}(l, .) \Delta \sum_{j=1}^{n_{1}}<Y_{j}^{(1)}, Y_{j}^{(1)}>(l, .) \\
& =\sum_{l=1}^{i} U^{n_{1} 2}(l, .) \sum_{j=1}^{n_{1}}\left[\Delta A_{j}^{(1)}(l, .)-\Delta A_{j}^{(1)^{2}}(l, .)\right] \\
& =\sum_{l=1}^{i} U^{n_{1} 2}(l, .) \sum_{j=1}^{n_{1}}\left[V_{j}^{(1)}(l, .) h^{(1)}(l)-V_{j}^{(1)^{2}}(l, .) h^{(1)}{ }^{2}(l)\right] \\
& =\sum_{l=1}^{i} U^{n_{1} 2}(l, .)\left[h^{(1)}(l, .) V^{n_{1}}(l, .)-h^{(1)}(l, .) V^{n_{1}}(l, .)\right] \\
& =\sum_{l=1}^{i} U^{n_{1} 2}(l, .) V^{n_{1}}(l, .) h^{(1)}(l)\left[1-h^{(1)}(l)\right]
\end{aligned}
$$

Assim,

$$
\begin{aligned}
E\left[\left(U^{n_{1} 2} .<Y^{n_{1}}, Y^{n_{1}}>\right)(i, .)\right] & =E\left[\sum_{l=1}^{i} U^{n_{1} 2}(l, .) V^{n_{1}}(l, .) h^{(1)}(l)\left[1-h^{(1)}(l)\right]\right] \\
& =\sum_{l=1}^{i} h^{(1)}(l)\left[1-h^{(1)}(l)\right] E\left[U^{n_{1} 2}(l, .) V^{n_{1}}(l, .)\right]
\end{aligned}
$$

Se $G_{l}$ denota o limite de $E\left[U^{n_{1} 2} V^{n_{1}}(l,).\right]$ quando $n_{1} \rightarrow \infty$, para $l=1, \ldots, i$, então obtemos que

$$
E\left[\sum_{j=1}^{n_{1}}\left[a_{j}(i)\right]^{2}\right] \rightarrow \sum_{l=1}^{i}\left[h^{(1)}(l)-h^{(1)^{2}}(l)\right] G_{l}, \quad \text { quando } n_{1} \rightarrow \infty .
$$

Desde que o processo $R(i)$ é um martingale uniformemente integrável concluímos que

$$
\sum_{j=1}^{n_{1}}\left[a_{j}(i)\right]^{2} \rightarrow \sum_{l=1}^{i}\left[h^{(1)}(l)-h^{(1)^{2}}(l)\right] G_{l} \text { quando } n_{1} \rightarrow \infty .
$$

Teorema 4.1. Temos que

$$
R_{n_{1}}(i)=\sum_{j=1}^{n_{1}} a_{j}(i)=\sum_{l=1}^{i} U^{n_{1}}(l, .) \Delta Y^{n_{1}}(l, .)
$$

converge em distribuição para uma variávela aleatória normal com média 0 e variância $\sigma^{2}(i)$. 
Demonstração. Pela Proposição 4.1 concluímos que o processo $\left\{a_{j}(i): 0 \leq j \leq n_{1}\right\}$ é um martingale array difference. Além disso, utilizando os Lemas 4.1 e 4.2, concluímos pelo Teorema 2.5, item (b), de Helland (1982) que $R_{n_{1}}(i)$ converge em distribuição para uma variável aleatória normal com média 0 e variância $\sigma^{2}(i)$.

Corolário 4.1. Ao denotarmos

$$
U^{n_{1}}(l, .)= \begin{cases}\frac{\sqrt{n_{1}}}{V^{n_{1}}(s, .)}, & l=s \\ 0, & l \neq s\end{cases}
$$

para $l, s=1, \ldots, i$, obtemos que $\sqrt{n_{1}}\left[\hat{h}^{n_{1}}(s)-h^{(1)}(s)\right]$ converge em distribuição para uma variável aleatória normal com média 0 e variância

$$
\sigma_{h}^{2}(s)=\frac{\left[h^{(1)}(s)-h^{(1)^{2}}(s)\right]}{\theta^{(1)}(s)}
$$

Demonstração. Para $U^{n_{1}}(s,)=.\frac{\sqrt{n_{1}}}{V^{n_{1}}(s, .)}$ e $U^{n_{1}}(l,)=$.0 para todo $l \neq s$, o processo $R_{n_{1}}(s)$ é dado por

$$
\begin{aligned}
R_{n_{1}}(s)=U^{n_{1}}(s, .) \Delta Y^{n_{1}}(s, .) & =\frac{\sqrt{n_{1}}}{V^{n_{1}}(s, .)} \Delta Y^{n_{1}}(s, .)=\frac{\sqrt{n_{1}}}{V^{n_{1}}(s, .)}\left[\Delta N^{n_{1}}(s, .)-\Delta A^{n_{1}}(s, .)\right] \\
& =\frac{\sqrt{n_{1}}}{V^{n_{1}}(s, .)}\left[\Delta N^{n_{1}}(s, .)-V^{n_{1}}(s, .) h^{(1)}(l)\right] \\
& =\sqrt{n_{1}}\left[\frac{\Delta N^{n_{1}}(s, .)}{V^{n_{1}}(s, .)}-h^{(1)}(s)\right] \\
& =\sqrt{n_{1}}\left[\hat{h}^{n_{1}}(s)-h^{(1)}(s)\right]
\end{aligned}
$$

Além disso,

$$
E\left[U^{n_{1} 2}(s, .) V^{n_{1}}(s, .)\right]=E\left[\frac{n_{1}}{V^{n_{1}}(s, .)}\right] \rightarrow \frac{1}{\theta_{s}^{(1)}}
$$

Portanto, pelo Teorema 4.1 temos que

$$
\sqrt{n_{1}}\left[\hat{h}^{n_{1}}(s)-h^{(1)}(s)\right]
$$

converge em distribuição para uma variável aleatória com média 0 e variância $\frac{\left[h^{(1)}(s)-h^{(1)^{2}}(s)\right]}{\theta^{(1)}(s)}$. 
Corolário 4.2. Tomando $U^{n_{1}}(l,)=.\frac{\sqrt{n_{1}}}{V^{n_{1}}(l, .)}$, para $l=1, \ldots, i$, obtemos que $\sqrt{n_{1}}\left[\hat{H}^{n_{1}}(l)-\right.$ $\left.H^{(1)}(l)\right]$ converge em distribuição para uma variável aleatória com média 0 e variância

$$
\sigma_{H}^{2}(i)=\sum_{l=1}^{i} \frac{\left[h^{(1)}(l)-h^{(1)^{2}}(l)\right]}{\theta^{(1)}(l)} .
$$

Demonstração. Para $U^{n_{1}}(l,)=.\frac{\sqrt{n_{1}}}{V^{n_{1}}(s, .)}$ para todo $l=1, \ldots, i$, o processo $R_{n_{1}}(i)$ é dado por

$$
\begin{aligned}
R_{n_{1}}(i)=\sum_{l=1}^{i} U^{n_{1}}(l, .) \Delta Y^{n_{1}}(l, .) & =\sum_{l=1}^{i} \frac{\sqrt{n_{1}}}{V^{n_{1}}(l, .)} \Delta Y^{n_{1}}(l, .)=\sum_{l=1}^{i} \frac{\sqrt{n 1}}{V^{n_{1}}(l, .)}\left[\Delta N^{n_{1}}(l, .)-\Delta A^{n_{1}}(l, .)\right] \\
& =\sum_{l=1}^{i} \frac{\sqrt{n_{1}}}{V^{n_{1}}(l, .)}\left[\Delta N^{n_{1}}(l, .)-V^{n_{1}}(l, .) h^{(1)}(l)\right] \\
& =\sum_{l=1}^{i} \sqrt{n_{1}}\left[\frac{\Delta N^{n_{1}}(l, .)}{V^{n_{1}}(l, .)}-h^{(1)}(l)\right] \\
& =\sum_{l=1}^{i} \sqrt{n_{1}}\left[\hat{h}^{n_{1}}(l)-h^{(1)}(l)\right] \\
& =\sqrt{n_{1}}\left[\hat{H}^{n_{1}}(i)-H^{(1)}(i)\right]
\end{aligned}
$$

Além disso,

$$
E\left[U^{n_{1} 2}(l, .) V^{n_{1}}(l, .)\right]=E\left[\frac{n_{1}}{V^{n_{1}}(l, .)}\right] \rightarrow \frac{1}{\theta^{(1)}(l)}, \text { quando } n_{1} \rightarrow \infty, \forall l=1, \ldots, i .
$$

Portanto, pelo Teorema 4.1 temos que

$$
\sqrt{n_{1}}\left[\hat{H}^{n_{1}}(i)-H^{(1)}(i)\right]
$$

converge em distribuição para uma variável aleatória com média 0 e variância $\sum_{l=1}^{i} \frac{\left[h^{(1)}(l)-h^{(1)^{2}}(l)\right]}{\theta^{(1)}(l)}$.

\subsection{Duas Amostras}

Consideremos duas amostras aleatórias simples independentes de tamanho $n_{1}$ e $n_{2}$, dadas, respectivamente, por $\left(\left(W_{1}^{(1)}, C_{1}^{(1)}\right), \ldots,\left(W_{n_{p}}^{(1)}, C_{n_{1}}^{(1)}\right)\right)$ e $\left(\left(W_{1}^{(2)}, C_{1}^{(2)}\right), \ldots,\left(W_{n_{2}}^{(2)}, C_{n_{2}}^{(2)}\right)\right)$.

A seguir, iremos desenvolver o processo e a filtragem correspondente utilizados no estudo de convergência. O processo será construído termo a termo. A filtragem será formada por álgebras encaixantes construídas conforme é construído cada termo do processo. 
Suponha, sem perda de generalidade, que $n_{1} \geq n_{2}$ e considere $i \in\{1, \ldots, k\}, k \in \mathbb{N}$, fixo. No ponto inicial (zero), a álgebra é dada por

$$
\begin{aligned}
& \tau_{i}(0)=\text { álgebra gerada por\{ } V^{n_{1}}(1, .), V^{n_{2}}(1, .), V_{1}^{(1)}(1, .), V_{1}^{(2)}(1, .) ; \ldots \text {; } \\
& \left.V^{n_{1}}(i, .) \quad, V^{n_{2}}(i, .), V_{1}^{(1)}(i, .), V_{1}^{(2)}(i, .)\right\}
\end{aligned}
$$

e o termo correspondente ao ponto zero é nulo $\left(a_{0}=0\right)$. Assim, consideraremos $a_{1}$ como sendo o primeiro termo do processo que será construído. Seja $U^{n_{1}, n_{2}}(l,),. l=1, \ldots, i$, uma variável aleatória com respeito à álgebra $\tau_{i}(0)$, com as seguintes propriedades

(a) Para $n_{1}$ e $n_{2}$ fixos o processo $U^{n_{1}, n_{2}}$ é previsível na filtragem produto $\left\{\mathcal{B}^{N^{n_{1}}}\right\} \times\left\{\mathcal{B}^{N^{n_{2}}}\right\}$.

(b) $\mathrm{E}\left[\left|\frac{U^{n_{1}, n_{2}}(l, .)}{V^{n_{p}}(l, .)}\right|\right] \rightarrow 0$, quando $n_{1}, n_{2} \rightarrow \infty$, para $p=1,2$ e $l=1, \ldots, i$.

(c) $\mathrm{E}\left[\frac{U^{n_{1}, n_{2} 2}(l, .)}{V^{n_{p}}(l, .)}\right] \rightarrow G_{l}^{(p)}$, quando $n_{1}, n_{2} \rightarrow \infty$, para $G^{(p)}>0, p=1,2, l=1, \ldots, i$.

De forma geral, assumimos que

$$
\frac{n_{1}+n_{2}}{n_{p}} \rightarrow u_{p}
$$

para $p=1,2$ e $u_{p} \in \mathbb{R}$, quando $n_{1}, n_{2} \rightarrow \infty$. Definimos o primeiro termo do processo

$$
a_{1}=\sum_{l=1}^{i} U^{n_{1}, n_{2}}(l, .)\left[\frac{\Delta Y_{1}^{(1)}(l, .)}{V^{n_{1}}(l, .)}-\frac{\Delta Y_{1}^{(2)}(l, .)}{V^{n_{2}}(l, .)}\right]
$$

e a álgebra nesse ponto é

$$
\begin{aligned}
& \tau_{i}(1)=\text { álgebra gerada por }\left\{\quad V^{n_{1}}(1, .), V^{n_{2}}(1, .), V_{1}^{(1)}(1, .), V_{2}^{(1)}(1, .), V_{1}^{(2)}(1, .), V_{2}^{(2)}(1, .),\right. \\
& \Delta N_{1}^{(1)}(1, .), \Delta N_{1}^{(2)}(1, .) ; \ldots ; V^{n_{1}}(i, .), V^{n_{2}}(i, .), V_{1}^{(1)}(i, .), \\
& V_{2}^{(1)}(i, .), V_{1}^{(2)}(i, .), V_{2}^{(2)}(i, .), \Delta N_{1}^{(1)}(i, .), \Delta N_{1}^{(2)}(i, .) \text {; } \\
& \left.C_{1}^{(1)}, \ldots, C_{n_{1}}^{(1)}, C_{1}^{(2)}, \ldots, C_{n_{2}}^{(2)}\right\} \text {. }
\end{aligned}
$$

O segundo termo é

$$
a_{2}=\sum_{l=1}^{i} U^{n_{1}, n_{2}}(l, .)\left[\frac{\Delta Y_{2}^{(1)}(l, .)}{V^{n_{1}}(l, .)}-\frac{\Delta Y_{2}^{(2)}(l, .)}{V^{n_{2}}(l, .)}\right]
$$


e a álgebra nesse ponto é

$$
\begin{aligned}
\tau_{i}(2)=\text { álgebra gerada por\{ } & V^{n_{1}}(1, .), V^{n_{2}}(1, .), V_{1}^{(1)}(1, .), V_{2}^{(1)}(1, .), V_{3}^{(1)}(1, .), V_{1}^{(2)}(1, .), \\
& V_{2}^{(2)}(1, .), V_{3}^{(2)}(1, .), \Delta N_{1}^{(1)}(1, .), \Delta N_{2}^{(1)}(1, .), \Delta N_{1}^{(2)}(1, .), \Delta N_{2}^{(2)}(1, .) ; \ldots ; \\
& V^{n_{1}}(i, .), V^{n_{2}}(i, .), V_{1}^{(1)}(i, .), V_{2}^{(1)}(i, .), V_{3}^{(1)}(i, .), V_{1}^{(2)}(i, .), V_{2}^{(2)}(i, .), \\
& V_{3}^{(2)}(i, .), \Delta N_{1}^{(1)}(i, .), \Delta N_{2}^{(1)}(i, .), \Delta N_{1}^{(2)}(i, .), \Delta N_{2}^{(2)}(i, .) ; \\
& \left.C_{1}^{(1)}, \ldots, C_{n_{1}}^{(1)}, C_{1}^{(2)}, \ldots, C_{n_{2}}^{(2)}\right\} .
\end{aligned}
$$

Definimos de forma análoga todos os termos até o termo de número $n_{2}$. Assim, para $s=1, \ldots, n_{2}$, os termos do processo são definidos conforme a equação 4.13

$$
a_{s}=\sum_{l=1}^{i} U^{n_{1}, n_{2}}(l, .)\left[\frac{\Delta Y_{s}^{(1)}(l, .)}{V^{n_{1}}(l, .)}-\frac{\Delta Y_{s}^{(2)}(l, .)}{V^{n_{2}}(l, .)}\right]
$$

A álgebra também é definida analogamente até o ponto $n_{2}-1$. Portanto, para $s=1, \ldots, n_{2}-$ 1 ,

$$
\begin{aligned}
& \tau_{i}(s)=\text { álgebra gerada por }\left\{\quad V^{n_{1}}(1, .), V^{n_{2}}(1, .), V_{1}^{(1)}(1, .), \ldots, V_{s+1}^{(1)}(1, .), V_{1}^{(2)}(1, .), \ldots, V_{s+1}^{(2)}(1, .),\right. \\
& \Delta N_{1}^{(1)}(1, .), \ldots, \Delta N_{s}^{(1)}(1, .), \Delta N_{1}^{(2)}(1, .), \ldots, \Delta N_{s}^{(2)}(1, .) ; \ldots \text {; } \\
& V^{n_{1}}(i, .), V^{n_{2}}(i, .), V_{1}^{(1)}(i, .), \ldots, V_{s+1}^{(1)}(i, .), V_{1}^{(2)}(i, .), \ldots, V_{s+1}^{(2)}(i, .) \text {, } \\
& \Delta N_{1}^{(1)}(i, .), \ldots, \Delta N_{s}^{(1)}(i, .), \Delta N_{1}^{(2)}(i, .), \ldots, \Delta N_{s}^{(2)}(i, .) \text {; } \\
& \left.C_{1}^{(1)}, \ldots, C_{n_{1}}^{(1)}, C_{1}^{(2)}, \ldots, C_{n_{2}}^{(2)}\right\}
\end{aligned}
$$

No entanto, no ponto $n_{2}$, a álgebra é definida de forma diferente

$$
\begin{aligned}
& \tau_{i}\left(n_{2}\right)=\text { álgebra gerada por }\left\{\quad V^{n_{1}}(1, .), V^{n_{2}}(1, .), V_{1}^{(1)}(1, .), \ldots, V_{n_{2}+1}^{(1)}(1, .), V_{1}^{(2)}(1, .), \ldots, V_{n_{2}}^{(2)}(1, .),\right. \\
& \Delta N_{1}^{(1)}(1, .), \ldots, \Delta N_{n_{2}}^{(1)}(1, .), \Delta N_{1}^{(2)}(1, .), \ldots, \Delta N_{n_{2}}^{(2)}(1, .) ; \ldots \text {; } \\
& V^{n_{1}}(i, .), V^{n_{2}}(i, .), V_{1}^{(1)}(i, .), \ldots, V_{n_{2}+1}^{(1)}(i, .), V_{1}^{(2)}(i, .), \ldots, V_{n_{2}}^{(2)}(i, .) \text {, } \\
& \Delta N_{1}^{(1)}(i, .), \ldots, \Delta N_{n_{2}}^{(1)}(i, .), \Delta N_{1}^{(2)}(i, .), \ldots, \Delta N_{n_{2}}^{(2)}(i, .) ; \\
& \left.C_{1}^{(1)}, \ldots, C_{n_{1}}^{(1)}, C_{1}^{(2)}, \ldots, C_{n_{2}}^{(2)}\right\} \text {. }
\end{aligned}
$$

Como $n_{1}>n_{2}$, a partir do ponto $n_{2}+1$ não existem elementos que correspondem à amostra $X^{(2)}$. Portanto, para $t=n_{2}+1, \ldots, n_{1}$,

$$
a_{t}=\sum_{l=1}^{i} U^{n_{1}, n_{2}}(l, .)\left[\frac{\Delta Y_{t}^{(1)}(l, .)}{V^{n_{1}}(l, .)}\right] .
$$


Para $t=n_{2}+1, \ldots, n_{1}-1$, a álgebra é dada conforme a equação 4.14. A álgebra para o último termo difere das demais e é definida conforme a equação 4.16 .

$$
\begin{aligned}
& \tau_{i}(t)=\text { álgebra gerada por }\left\{\quad V^{n_{1}}(1, .), V^{n_{2}}(1, .), V_{1}^{(1)}(1, .), \ldots, V_{n_{2}}^{(1)}(1, .), V_{1}^{(2)}(1, .), \ldots, V_{t+1}^{(2)}(1, .),\right. \\
& \Delta N_{1}^{(1)}(1, .), \ldots, \Delta N_{n_{2}}^{(1)}(1, .), \Delta N_{1}^{(2)}(1, .), \ldots, \Delta N_{t}^{(2)}(1, .) ; \ldots ; \\
& V^{n_{1}}(i, .), V^{n_{2}}(i, .), V_{1}^{(1)}(i, .), \ldots, V_{n_{2}}^{(1)}(i, .), V_{1}^{(2)}(i, .), \ldots, V_{t+1}^{(2)}(i, .), \\
& \Delta N_{1}^{(1)}(i, .), \ldots, \Delta N_{n_{2}}^{(1)}(i, .), \Delta N_{1}^{(2)}(i, .), \ldots, \Delta N_{t}^{(2)}(i, .) ; \\
& \left.C_{1}^{(1)}, \ldots, C_{n_{1}}^{(1)}, C_{1}^{(2)}, \ldots, C_{n_{2}}^{(2)}\right\} \text {. }
\end{aligned}
$$

$\tau_{i}\left(n_{1}\right)=$ álgebra gerada por $\left\{\quad V^{n_{1}}(1,),. V^{n_{2}}(1,),. V_{1}^{(1)}(1,),. \ldots, V_{n_{1}}^{(1)}(1,),. V_{1}^{(2)}(1,),. \ldots, V_{n_{1}}^{(2)}(1,)\right.$,

$$
\begin{aligned}
& \Delta N_{1}^{(1)}(1, .), \ldots, \Delta N_{n_{1}}^{(1)}(1, .), \Delta N_{1}^{(2)}(1, .), \ldots, \Delta N_{n_{1}}^{(2)}(1, .) ; \ldots ; \\
& V^{n_{1}}(i, .), V^{n_{2}}(i, .), V_{1}^{(1)}(i, .), \ldots, V_{n_{1}}^{(1)}(i, .), V_{1}^{(2)}(i, .), \ldots, V_{n_{1}}^{(2)}(i, .), \\
& \Delta N_{1}^{(1)}(i, .), \ldots, \Delta N_{n_{1}}^{(1)}(i, .), \Delta N_{1}^{(2)}(i, .), \ldots, \Delta N_{n_{1}}^{(2)}(i, .) ; \\
& \left.C_{1}^{(1)}, \ldots, C_{n_{1}}^{(1)}, C_{1}^{(2)}, \ldots, C_{n_{2}}^{(2)}\right\} .
\end{aligned}
$$

Consideremos as soma parciais $P_{j}^{n_{1}, n_{2}}(i)$ como sendo a soma dos termos $a_{0}, a_{1}, \ldots, a_{j}$ para $0 \leq j \leq n_{1}$ e $i \in\{1, \ldots, k\}$ fixo,

$$
P_{j}^{n_{1}, n_{2}}(i)=a_{0}+a_{1}+\ldots+a_{j}
$$

e seja $\left\{\tau_{i}\right\}$ a filtragem formada pelas álgebras encaixantes $\tau_{i}(j)$ com $0 \leq j \leq n_{1}$. Com isso, para $i \in\{1, \ldots, k\}$ fixo, definimos o processo estocástico

$$
P^{n_{1}, n_{2}}(i)=\left\{P_{0}^{n_{1}, n_{2}}(i), P_{1}^{n_{1}, n_{2}}(i), \ldots, P_{n_{1}}^{n_{1}, n_{2}}(i)\right\}
$$

que é adaptado à filtragem $\left\{\tau_{i}\right\}$ por construção.

Proposição 4.2. O processo estocástico $P^{n_{1}, n_{2}}(i)$, para $i \in\{1, \ldots, k\}$ fixo, é um martingale com respeito à filtragem $\left\{\tau_{i}\right\}$.

Demonstração. Por construção o processo $P^{n_{1}, n_{2}}(i)$ é adaptado à filtragem $\left\{\tau_{i}\right\}$. Com isso, basta provar que

$$
\mathrm{E}\left[P_{m}^{n_{1}, n_{2}}(i) \mid \tau_{i}(m-1)\right]=P_{m-1}^{n_{1}, n_{2}}(i)
$$


No entanto, pela definição das somas parciais dada pela equação $4.17, P_{j}^{n_{1}, n_{2}}(i)-P_{j-1}^{n_{1}, n_{2}}(i)=a_{j}$, para todo $j=1, \ldots n_{1}$. Com isso, basta mostrar que

$$
\mathrm{E}\left[a_{j} \mid \tau_{i}(j-1)\right]=0 .
$$

Para $1 \leq j \leq n_{2}$, temos que

$$
\begin{aligned}
\mathrm{E}\left[a_{j} \mid \tau_{i}(j-1)\right] & =\mathrm{E}\left[\sum_{l=1}^{i} U^{n_{1}, n_{2}}(l, .)\left(\frac{\Delta Y_{j}^{(1)}(l, .)}{V^{n_{1}}(l, .)}-\frac{\Delta Y_{j}^{(2)}(l, .)}{V^{n_{2}}(l, .)}\right) \mid \tau_{i}(j-1)\right] \\
& =\sum_{l=1}^{i} U^{n_{1}, n_{2}}(l, .)\left\{\frac{1}{V^{n_{1}}(l, .)} \mathrm{E}\left[\Delta N_{j}^{(1)}(l .)-\Delta A_{j}^{(1)}(l, .) \mid \tau_{i}(j-1)\right]\right. \\
& \left.-\frac{1}{V^{n_{2}}(l, .) n_{1}} \mathrm{E}\left[\Delta N_{j}^{(2)}(l .)-\Delta A_{j}^{(2)}(l, .) \mid \tau_{i}(j-1)\right]\right\}
\end{aligned}
$$

Observe que $\Delta A_{j}^{(p)}(l,$.$) é mensurável em \tau_{i}(j-1)$ e $\Delta N_{j}^{(p)}(l,$.$) condicionado a V_{j}^{(p)}(l,$.$) é in-$ dependente de todos os outros termos da filtragem, $\tau_{i}(j-1)$ para $1 \leq j \leq n_{2}$ e $p=1,2$. Utilizando essas propriedades, temos que

$$
\begin{aligned}
E\left[\sum_{l=1}^{i} U^{n_{1}, n_{2}}(l, .)\right. & \left.\left(\frac{\Delta Y_{j}^{(1)}(l, .)}{V^{n_{1}}(l, .)}-\frac{\Delta Y_{j}^{(2)}(l, .)}{V^{n_{2}}(l, .)}\right) \mid \tau_{i}(j-1)\right] \\
= & \sum_{l=1}^{i} U^{n_{1}, n_{2}}(l, .)\left\{\frac{1}{V^{n_{1}}(l, .) n_{2}} \mathrm{E}\left[\Delta N_{j}^{(1)}(l .) \mid V_{j}^{(1)}(l, .)\right]-V_{j}^{(1)}(l, .) h^{(1)}(l)\right\} \\
& -\sum_{l=1}^{i} U^{n_{1}, n_{2}}(l, .)\left\{\frac{1}{V^{n_{2}}(l, .) n_{1}} \mathrm{E}\left[\Delta N_{j}^{(2)}(l .) \mid V_{j}^{(2)}(l, .)\right]-V_{j}^{(2)}(l, .) h^{(2)}(l)\right\} \\
= & 0
\end{aligned}
$$

pois $\Delta N_{j}^{(p)}(l,) \mid. V_{j}^{(p)}(l,$.$) tem distribuição binomial com número de ensaios V_{j}^{(p)}(l,$.$) e probabil-$ idade $h^{(p)}(l)$, para $p=1,2$. Para $n_{2}<j \leq n_{1}$ os termos do processo são dados por

$$
a_{j}=\sum_{l=1}^{i} U^{n_{1}, n_{2}}(l, .) \frac{\Delta Y_{j}^{(1)}(l, .)}{V^{n_{1}}(l, .)}
$$

As propriedades verificadas para o primeiro caso também são válidas para esse caso. Assim, de forma análoga ao realizado anteriormente, temos que

$$
\mathrm{E}\left[\sum_{l=1}^{i} U^{n_{1}, n_{2}}(l, .) \frac{\Delta Y_{j}^{(1)}(l, .)}{V^{n_{1}}(l, .)} \mid \tau_{i}(j-1)\right]=0
$$


Da Proposição 2.2, concluímos que o processo $\left\{a_{j}: 0 \leq j \leq n_{1}\right\}$ é um martingale array difference em relaçao a filtragem $\left\{\tau_{i}\right\}$.

Lema 4.3. Considere os termos $a_{j}, j=1, \ldots, n_{1}$. A seguinte propriedade é válida

$$
E\left[\max _{1 \leq j \leq n_{1}}\left|a_{j}\right|\right] \rightarrow 0, \quad \text { quando } n_{1}, n_{2} \rightarrow \infty .
$$

Demonstração. Para $1 \leq j \leq n_{2}$,

$$
a_{j}=\sum_{l=1}^{i} U^{n_{1}, n_{2}}(l, .)\left[\frac{\Delta Y_{j}^{(1)}(l .)}{V^{n_{1}}(l, .)}-\frac{\Delta Y_{j}^{(2)}(l, .)}{V^{n_{2}}(l, .)}\right] .
$$

Utilizando propriedades de módulo e máximo, temos que

$$
\begin{aligned}
\max _{1 \leq j \leq n_{2}}\left|\sum_{l=1}^{i} U^{n_{1}, n_{2}}(l, .)\left[\frac{\Delta Y_{j}^{(1)}(l .)}{V^{n_{1}}(l, .)}-\frac{\Delta Y_{j}^{(2)}(l, .)}{V^{n_{2}}(l, .)}\right]\right| & \leq \max _{1 \leq j \leq n_{2}} \sum_{l=1}^{i}\left|U^{n_{1}, n_{2}}(l, .)\left[\frac{\Delta Y_{j}^{(1)}(l .)}{V^{n_{1}}(l, .)}-\frac{\Delta Y_{j}^{(2)}(l, .)}{V^{n_{2}}(l, .)}\right]\right| \\
& \leq \sum_{l=1}^{i} \max _{1 \leq j \leq n_{2}}\left|U^{n_{1}, n_{2}}(l, .)\left[\frac{\Delta Y_{j}^{(1)}(l .)}{V^{n_{1}}(l, .)}-\frac{\Delta Y_{j}^{(2)}(l, .)}{V^{n_{2}}(l, .)}\right]\right|
\end{aligned}
$$

Com isso,

$$
\begin{aligned}
\mathrm{E}\left[\max _{1 \leq j \leq n_{2}}\left|\sum_{l=1}^{i} U^{n_{1}, n_{2}}(l, .)\left[\frac{\Delta Y_{j}^{(1)}(l, .)}{V^{n_{1}}(l, .)}-\frac{\Delta Y_{j}^{(2)}(l, .)}{V^{n_{2}}(l, .)}\right]\right|\right] \\
\leq \mathrm{E}\left[\sum_{l=1}^{i} \max _{1 \leq j \leq n_{2}}\left|U^{n_{1}, n_{2}}(l, .)\left[\frac{\Delta Y_{j}^{(1)}(l .)}{V^{n_{1}}(l, .)}-\frac{\Delta Y_{j}^{(2)}(l, .)}{V^{n_{2}}(l, .)}\right]\right|\right]
\end{aligned}
$$

Por outro lado,

$$
\begin{aligned}
& \left|U^{n_{1}, n_{2}}(l, .)\left[\frac{\Delta Y_{j}^{(1)}(l .)}{V^{n_{1}}(l, .)}-\frac{\Delta Y_{j}^{(2)}(l .)}{V^{n_{2}}(l, .)}\right]\right| \\
& \quad=\left|U^{n_{1}, n_{2}}(l, .)\left[\frac{\Delta N_{j}^{(1)}(l, .)-\Delta A_{j}^{(1)}(l, .)}{V^{n_{1}}(l, .)}-\frac{\Delta N_{j}^{(2)}(l .)-\Delta A_{j}^{(2)}(l, .)}{V^{n_{2}}(l, .)}\right]\right| \\
& \leq\left|U^{n_{1}, n_{2}}(l, .) \frac{\Delta N_{j}^{(1)}(l .)-\Delta A_{j}^{(1)}(l, .)}{V^{n_{1}}(l, .)}\right|+\left|U^{n_{1}, n_{2}}(l, .) \frac{\Delta N_{j}^{(2)}(l .)-\Delta A_{j}^{(2)}(l, .)}{V^{n_{2}}(l, .)}\right| \\
& \leq\left|\frac{U^{n_{1}, n_{2}}(l, .)}{V^{n_{1}}(l, .)}\right|\left(\left|\Delta N_{j}^{(1)}(l .)\right|+\left|\Delta A_{j}^{(1)}(l, .)\right|\right)+\left|\frac{U^{n_{1}, n_{2}}(l, .)}{V^{n_{1}}(l, .)}\right|\left(\left|\Delta N_{j}^{(2)}(l .)\right|+\left|\Delta A_{j}^{(2)}(l, .)\right|\right) \\
& \quad \leq 2\left|\frac{U^{n_{1}, n_{2}}(l, .)}{V^{n_{1}}(l, .)}\right|+2\left|\frac{U^{n_{1}, n_{2}}(l, .)}{V^{n_{2}}(l, .)}\right|
\end{aligned}
$$

Das equações 4.24 e 4.24, obtemos que 
$\mathrm{E}\left[\max _{1 \leq j \leq n_{2}}\left|\sum_{l=1}^{i} U^{n_{1}, n_{2}}(l,).\left[\frac{\Delta Y_{j}^{(1)}(l, .)}{V^{n_{1}}(l, .)}-\frac{\Delta Y_{j}^{(2)}(l, .)}{V^{n_{2}}(l, .)}\right]\right|\right]$

$$
\begin{aligned}
& \leq \mathrm{E}\left[\max _{1 \leq j \leq n_{2}} 2 i\left|\frac{U^{n_{1}, n_{2}}(l, .)}{V^{n_{1}}(l, .)}\right|+2 i\left|\frac{U^{n_{1}, n_{2}}(l, .)}{V^{n_{2}}(l, .)}\right|\right] \\
& =2 i \mathrm{E}\left[\left|\frac{U^{n_{1}, n_{2}}(l, .)}{V^{n_{1}}(l, .)}\right|\right]+2 i \mathrm{E}\left[\left|\frac{U^{n_{1}, n_{2}}(l, .)}{V^{n_{2}}(l, .)}\right|\right]
\end{aligned}
$$

para $i \in\{1, \ldots, k\}$ fixo, com $k \in \mathbb{N}$. Por hipóteste $E\left[\left|\frac{U^{n_{1}, n_{2}}(l, .)}{V^{n_{1}}(l, .)}\right|\right] \rightarrow 0$ e $E\left[\left|\frac{U^{n_{1}, n_{2}}(l, .)}{V^{n_{2}}(l, .)}\right|\right] \rightarrow$ 0 quando $n_{1}, n_{2} \rightarrow \infty, \forall l$. Portanto,

$$
\mathrm{E}\left[\max _{1 \leq j \leq n_{2}}\left|\sum_{l=1}^{i} U^{n_{1}, n_{2}}(l, .)\left[\frac{\Delta Y_{j}^{(1)}(l .)}{V^{n_{1}}(l, .)}-\frac{\Delta Y_{j}^{(2)}(l, .)}{V^{n_{2}}(l, .)}\right]\right|\right] \rightarrow 0 \text {, quando } n_{1}, n_{2} \rightarrow \infty .
$$

Para $n_{2}<j \leq n_{1}$,

$$
a_{j}=\sum_{l=1}^{i} U^{n_{1}, n_{2}}(l, .) \frac{\Delta Y_{j}^{(1)}(l, .)}{V^{n_{1}}(l, .)} .
$$

De forma forma análoga ao caso anteorior obtemos as seguintes desigualdades

$$
\mathrm{E}\left[\max _{1 \leq j \leq n_{2}}\left|\sum_{l=1}^{i} U^{n_{1}, n_{2}}(l, .) \frac{\Delta Y_{j}^{(1)}(l, .)}{V^{n_{1}}(l, .)}\right|\right] \leq \mathrm{E}\left[\sum_{l=1}^{i} \max _{1 \leq j \leq n_{2}}\left|U^{n_{1}, n_{2}}(l, .) \frac{\Delta Y_{j}^{(1)}(l .)}{V^{n_{1}}(l, .)}\right|\right]
$$

e

$$
\left|U^{n_{1}, n_{2}}(l, .) \frac{\Delta Y_{j}^{(1)}(l .)}{V^{n_{1}}(l, .)}\right| \leq 2 \frac{U^{n_{1}, n_{2}}(l, .)}{V^{n_{1}}(l, .)}
$$

$\forall l=1, \ldots, i$.

Assim, concluímos que

$$
\mathrm{E}\left[\max _{1 \leq j \leq n_{2}}\left|\sum_{l=1}^{i} U^{n_{1}, n_{2}}(l, .) \frac{\Delta Y_{j}^{(1)}(l, .)}{V^{n_{1}}(l, .)}\right|\right] \rightarrow 0, \text { quando } n_{1}, n_{2} \rightarrow \infty .
$$

Lema 4.4. Consideremos o processo $\left\{a_{j}: 0 \leq j \leq n_{1}\right\}$ um martingale array difference como definimos anteriormente. Temos que

$$
\sum_{j=1}^{n_{1}} a_{j}^{2}(i) \rightarrow \sigma^{2}(i)
$$

em probabilidade, quando $n_{1}, n_{2} \rightarrow \infty$, em que

$$
\sigma^{2}(i)=\sum_{l=1}^{i}\left[1-h^{(1)}(l)\right] h^{(1)}(l) G_{l}^{1}+\sum_{l=1}^{i}\left[1-h^{(2)}(l)\right] h^{(2)}(l) G_{l}^{2} .
$$


Demonstração. Observe que a $\sum_{j=1}^{n_{1}} a_{j}(i)$ pode ser escrito da seguinte forma

$$
\sum_{j=1}^{n_{1}} a_{j}(i)=\sum_{j_{1}=1}^{n_{1}} \sum_{j_{2}=1}^{n_{2}}\left(\sum_{l=1}^{i} U^{n_{1}, n_{2}}(l, .)\left[\frac{\Delta Y_{j_{1}}^{(1)}(l, .)}{V^{n_{1}}(l, .) n_{2}}-\frac{\Delta Y_{j_{2}}^{(2)}(l, .)}{V^{n_{2}}(l, .) n_{1}}\right]\right)
$$

Conforme a Proposição 2.1 e pela equação 4.25 temos que

$$
\begin{aligned}
\mathrm{E}\left[\sum_{j=1}^{n_{1}} a_{j}{ }^{2}(i)\right] & =\mathrm{E}\left[\left(\sum_{l=1}^{n_{1}} a_{j}(i)\right)^{2}\right] \\
& =\mathrm{E}\left[\left(\sum_{m_{1}=1}^{n_{1}} \sum_{m_{2}=1}^{n_{2}} \sum_{l=1}^{i} U^{n_{1}, n_{2}}(l, .)\left[\frac{\Delta Y_{m}^{(1)}(l, .)}{V^{n_{1}}(l, .) n_{2}}-\frac{\Delta Y_{m}^{(2)}(l, .)}{V^{n_{2}}(l, .) n_{1}}\right]\right)^{2}\right] \\
& =\mathrm{E}\left[\left(\sum_{l=1}^{i} U^{n_{1}, n_{2}}(l, .)\left[\frac{\Delta Y^{n_{1}}(l, .)}{V^{n_{1}}(l, .)}-\frac{\Delta Y^{n_{2}}(l, .)}{V^{n_{2}}(l, .)}\right]\right)^{2}\right]
\end{aligned}
$$

Denotando por

$$
\Delta F(l, .)=\frac{\Delta Y^{n_{1}}(l, .)}{V^{n_{1}}(l, .)}-\frac{\Delta Y^{n_{2}}(l, .)}{V^{n_{2}}(l, .)}
$$

temos que

$$
\begin{aligned}
\mathrm{E}\left[\left(\sum_{l=1}^{i} U^{n_{1}, n_{2}}(l, .)\left[\frac{\Delta Y^{n_{1}}(l, .)}{V^{n_{1}}(l, .)}-\frac{\Delta Y^{n_{2}}(l, .)}{V^{n_{2}}(l, .)}\right]\right)^{2}\right] & =E\left[\left(\sum_{l=1}^{i} U^{n_{1}, n_{2}}(l, .) \Delta F(l, .)\right)^{2}\right] \\
& \left.=E\left[\left(U^{n_{1}, n_{2}} \cdot F\right)^{2}(i, .)\right]\right] \\
& =E\left[<\left(U^{n_{1}, n_{2}} \cdot F\right),\left(U^{n_{1}, n_{2}} . F\right)>(i, .)\right] \\
& =E\left[\left(U^{n_{1}, n_{2} 2} \cdot<F, F>\right)(i, .)\right]
\end{aligned}
$$

Por outro lado, temos que

$\left(U^{n_{1}, n_{2} 2} ;<F, F>\right)(i,$. 


$$
\begin{aligned}
& =\sum_{l=1}^{i} U^{n_{1}, n_{2} 2}(l, .) \Delta<F, F>(l, .) \\
& =\sum_{l=1}^{i} U^{n_{1}, n_{2} 2}(l, .)\left[\frac{1}{V^{n_{1}^{2}}(l, .)} \Delta<Y^{n_{1}}, Y^{n_{1}}>(l, .)+\frac{1}{V^{n_{2}}(l, .)} \Delta<Y^{n_{2}}, Y^{n_{2}}>(l, .)\right] \\
& =\sum_{l=1}^{i} U^{n_{1}, n_{2} 2}(l, .)\left[\frac{1}{V^{n_{1}}(l, .)} \Delta \sum_{m_{1}=1}^{n_{1}}<Y_{m_{1}}^{(1)}, Y_{m_{1}}^{(1)}>(l, .)+\frac{1}{V^{n_{2}}(l, .)} \Delta \sum_{m_{2}=1}^{n_{2}}<Y_{m_{2}}^{(2)}, Y_{m_{2}}^{(2)}>(l, .)\right] \\
& =\sum_{l=1}^{i} U^{n_{1}, n_{2} 2}(l, .)\left[\sum_{m_{1}=1}^{n_{1}} \frac{\Delta A_{m_{1}}^{(1)}(l, .)-\Delta A^{(1)}{ }_{m_{1}}^{2}(l, .)}{V^{n_{1} 2}(l, .)}+\frac{\sum_{m_{2}=1}^{n_{2}} \Delta A_{m_{2}}^{(2)}(l, .)-\Delta A_{m_{2}}^{(2)}(l, .)}{V^{n_{2} 2}(l, .)}\right] \\
= & \sum_{l=1}^{i} \frac{U^{n_{1}, n_{2} 2}(l, .)}{V^{n_{1}}(l, .)}\left[1-h^{(1)}(l)\right] h^{(1)}(l)+\sum_{l=1}^{i} \frac{U^{n_{1}, n_{2} 2}(l, .)}{V^{n_{2}}(l, .)}\left[1-h^{(2)}(l)\right] h^{(2)}(l)
\end{aligned}
$$

Assim,

$$
\begin{aligned}
\mathrm{E}\left[\sum_{j=1}^{n_{1}} a_{j}{ }^{2}(i)\right] & =\mathrm{E}\left[\sum_{l=1}^{i} \frac{U^{n_{1}, n_{2} 2}(l, .)}{V^{n_{1}}(l, .)}\left[1-h^{(1)}(l)\right] h^{(1)}(l)+\sum_{l=1}^{i} \frac{U^{n_{1}, n_{2} 2}(l, .)}{V^{n_{2}}(l, .)}\left[1-h^{(2)}(l)\right] h^{(2)}(l)\right] \\
& =\sum_{l=1}^{i}\left[1-h^{(1)}(l)\right] h^{(1)}(l) \mathrm{E}\left[\frac{U^{n_{1}, n_{2} 2}(l, .)}{V^{n_{1}}(l, .)}\right]+\sum_{l=1}^{i}\left[1-h^{(2)}(l)\right] h^{(2)}(l) \mathrm{E}\left[\frac{U^{n_{1}, n_{2} 2}(l, .)}{V^{n_{2}}(l, .)}\right]
\end{aligned}
$$

Se $G_{l}^{1}$ denota o limite de $\mathrm{E}\left[\frac{U^{n_{1}, n_{2}} 2(l, .)}{V^{n_{1}}(l, .)}\right]$ e $G_{l}^{2}$ denota o limite de $\mathrm{E}\left[\frac{U^{n_{1}, n_{2} 2}(l, .)}{V^{n_{2}}(l, .)}\right]$, quando $n_{1}, n_{2} \rightarrow \infty$, então obtemos que

$$
\mathrm{E}\left[\sum_{j=1}^{n_{1}} a_{j}{ }^{2}(i)\right] \rightarrow \sum_{l=1}^{i}\left[1-h^{(1)}(l)\right] h^{(1)}(l) G_{l}^{1}+\sum_{l=1}^{i}\left[1-h^{(2)}(l)\right] h^{(2)}(l) G_{l}^{2},
$$

quando $n_{1}, n_{2} \rightarrow \infty$. Conseqüentemente, pelo Teorema 2.7.2 de Stout (1970), temos que

$$
\sum_{j=1}^{n_{1}}{a_{j}}^{2}(i) \rightarrow \sum_{l=1}^{i}\left[1-h^{(1)}(l)\right] h^{(1)}(l) G_{l}^{1}+\sum_{l=1}^{i}\left[1-h^{(2)}(l)\right] h^{(2)}(l) G_{l}^{2}
$$

em probabilidade, quando $n_{1}, n_{2} \rightarrow \infty$.

Teorema 4.2. Temos que

$$
P_{n_{1}}^{n_{1}, n_{2}}(i)=\sum_{j=1}^{n_{1}} a_{j}(i)=\sum_{l=1}^{i} U^{n_{1}, n_{2}}(l, .)\left[\frac{\Delta Y^{n_{1}}(l, .)}{V^{n_{1}}(l, .)}-\frac{\Delta Y^{n_{2}}(l, .)}{V^{n_{2}}(l, .)}\right]
$$


converge em distribuição para uma variável aleatória normal com média 0 e variância $\sigma^{2}(i)$, quando $n_{1}, n_{2} \rightarrow \infty$.

Demonstração. Pela Proposição 4.2 concluímos que o processo $\left\{a_{j}(i): 0 \leq j \leq n_{1}\right\}$ é um martingale array difference. Além disso, utilizando os Lemas 4.1 e 4.2, concluímos pelo Teorema 2.5, item (b), de Helland (1982) que $P_{n_{1}}^{n_{1}, n_{2}}(i)$ converge em distribuição para uma variável aleatória com média 0 e variância $\sigma^{2}(i)$.

Corolário 4.3. Ao tomarmos

$$
U^{n_{1}, n_{2}}(l, .)=\left(\frac{n_{1}+n_{2}}{n_{1} n_{2}}\right)^{1 / 2} \frac{V^{n_{1}}(l, .) V^{n_{2}}(l, .)}{V^{n_{1}}(l, .)+V^{n_{2}}(l, .)},
$$

para $l=1, \ldots, i$, obtemos que

$$
\sum_{l=1}^{i} U^{n_{1}, n_{2}}(l, .)\left[\left(\hat{h}^{n_{1}}(l)-h^{(1)}(l)\right)-\left(\hat{h}^{n_{2}}(l)-h^{(2)}(l)\right)\right],
$$

converge em distribuição para uma variável aleatória com média 0 e variância $\sigma^{2}(i)$ dada por

$$
\sigma^{2}(i)=\sum_{l=1}^{i}\left(\left[1-\hat{h}^{n_{1}}(l)\right] \hat{h}^{n_{1}}(l) G_{l}^{1}+\left[1-\hat{h}^{n_{2}}(l)\right] \hat{h}^{n_{2}}(l) G_{l}^{2}\right) .
$$

Demonstração. Para $U^{n_{1}, n_{2}}(l,$.$) definido como na equação 4.28$, o processo $P_{n_{1}}^{n_{1}, n_{2}}(l)$ é dado por

$$
\begin{aligned}
P_{n_{1}}^{n_{1}, n_{2}}(i) & =\sum_{l=1}^{i} U^{n_{1}, n_{2}}(l, .)\left[\frac{\Delta Y^{n_{1}}(l, .)}{V^{n_{1}}(l, .)}-\frac{\Delta Y^{n_{2}}(l, .)}{V^{n_{2}}(l, .)}\right] \\
& =\sum_{l=1}^{i} U^{n_{1}, n_{2}}(l, .)\left[\frac{\Delta N^{n_{1}}(l, .)-\Delta A^{n_{1}}(l, .)}{V^{n_{1}}(l, .)}-\frac{\Delta N^{n_{2}}(l, .)-\Delta A^{n_{2}}(l, .)}{V^{n_{2}}(l, .)}\right] \\
& =\sum_{l=1}^{i} U^{n_{1}, n_{2}}(l, .)\left[\frac{\Delta N^{n_{1}}(l, .)-V^{n_{1}}(l, .) h^{(1)}(l)}{V^{n_{1}}(l, .)}-\frac{\Delta N^{n_{2}}(l, .)-V^{n_{2}}(l, .) h^{(2)}(l)}{V^{n_{2}}(l, .)}\right] \\
& =\sum_{l=1}^{i} U^{n_{1}, n_{2}}(l, .)\left[\left(\hat{h}^{n_{1}}(l)-h^{(1)}(l)\right)-\left(\hat{h}^{n_{2}}(l)-h^{(2)}(l)\right)\right]
\end{aligned}
$$

Além disso,

$$
\begin{aligned}
\left|\frac{U^{n_{1}, n_{2}}(l, .)}{V^{n_{1}}(l, .)}\right| & =\left(\frac{n_{1}+n_{2}}{n_{1} n_{2}}\right)^{1 / 2} \frac{V^{n_{2}}(l, .)}{V^{n_{1}}(l, .)+V^{n_{2}}(l, .)} \\
& \leq\left(\frac{n_{1}+n_{2}}{n_{1} n_{2}}\right)^{1 / 2} \\
& =\left(\frac{1}{n_{2}}+\frac{1}{n_{1}}\right)^{1 / 2}
\end{aligned}
$$


Assim, $\left|\frac{U^{n_{1}, n_{2}}(l, .)}{V^{n_{1}}(l, .)}\right|$ é limitado e converge para 0 em probabilidade quando $n_{1}, n_{2} \rightarrow \infty$. Portanto,

$$
\mathrm{E}\left[\left|\frac{U^{n_{1}, n_{2}}(l, .)}{V^{n_{1}}(l, .)}\right|\right] \rightarrow 0, \text { quando } n_{1}, n_{2} \rightarrow \infty .
$$

Da mesma forma, temos que

$$
\mathrm{E}\left[\left|\frac{U^{n_{1}, n_{2}}(l, .)}{V^{n_{2}}(l, .)}\right|\right] \rightarrow 0, \text { quando } n_{1}, n_{2} \rightarrow \infty .
$$

Por outro lado temos que

$$
\begin{aligned}
\frac{U^{n_{1}, n_{2} 2}(l, .)}{V^{n_{1}}(l, .)} & =\frac{n_{1}+n_{2}}{n_{1} n_{2}} \frac{V^{n_{1}}(l, .) V^{n_{2}}(l, .)}{\left(V^{n_{1}}(l, .)+V^{n_{2}}(l, .)\right)^{2}} \\
& =\frac{n_{1}+n_{2}}{n_{2}} \frac{V^{n_{1}}(l, .)}{n_{1}} \frac{V^{n_{2}}(l, .)}{\left(V^{n_{1}}(l, .)+V^{n_{2}}(l, .)\right)^{2}} \\
& \leq L \frac{n_{1}+n_{2}}{n_{2}} \frac{V^{n_{1}}(l, .)}{n_{1}}
\end{aligned}
$$

Como assumimos que

$$
\frac{n_{1}+n_{2}}{n_{p}} \rightarrow u_{p}
$$

$p=1,2$ e $u_{p} \in \mathbb{R}$, quando $n_{1}, n_{2} \rightarrow \infty$, ou seja, existe uma proporcionalidade entre $n_{1}$ e $n_{2}$, obtemos que existe o limite de

$$
\mathrm{E}\left[\frac{U^{n_{1}, n_{2} 2}(l, .)}{V^{n_{1}}(l, .)}\right] .
$$

Da mesma forma temos que existe o limite de

$$
\mathrm{E}\left[\frac{U^{n_{1}, n_{2} 2}(l, .)}{V^{n_{2}}(l, .)}\right] .
$$

Assim, como a Proposição 4.2 e o Lema 4.3 são satisfeitos, obtemos do Teorema 4.2 que

$$
\sum_{l=1}^{i} U^{n_{1}, n_{2}}(l, .)\left[\left(\hat{h}^{n_{1}}(l)-h^{(1)}(l)\right)-\left(\hat{h}^{n_{2}}(l)-h^{(2)}(l)\right)\right],
$$

converge em distribuição para uma variável aleatória com média 0 e variância $\sigma^{2}(i)$. 


\section{Capítulo 5}

\section{Testes de Hipóteses}

\subsection{Introdução}

Neste capítulo apresentamos testes de hipóteses para os parâmetros do modelo de intensidade multiplicativo para uma e duas populações. Os testes foram desenvolvidos utilizando o estudo de distribuição assintótica de integrais estocásticas apresentado no Capítulo 4.

A estatística de teste para comparação de duas populações com dados censurados, apresentada neste capítulo, foi proposta por Mantel (1966) e é baseada na estatística de MantelHaenszel para tabelas de contigências estratificadas. Esta estatística ficou conhecida como estatística de logrank e é provavelmente a mais utilizada como estatística de teste para comparação de duas populações com dados censurados.

\subsection{Teste de Hipóteses para uma População}

Nesta seção, apresentamos testes de hipóteses para os parâmetros do modelo de intensidade multiplicativo para uma população.

Consideremos uma amostra aleatória simples de tamanho $n, X^{(1)}=\left(\left(W_{1}^{(1)}, C_{1}^{(1)}\right), \ldots,\left(W_{n}^{(1)}, C_{n}^{(1)}\right)\right)$, e a teoria definida e desenvolvida no Capítulo 3. Como resultado do Corolário 4.1, temos que

$$
\sqrt{n}\left[\hat{h}^{n}(l)-h^{(1)}(l)\right]
$$

tem distribuição assintóticamente normal com média 0 e variância $\frac{\left[h^{(1)}(l)-h^{(1)^{2}}(l)\right]}{\theta^{(1)}(l)}$, para 
$l=1, \ldots, i$ e $i \in\{1, \ldots, k\}$, em que $\theta^{(1)}(l)=\eta^{(1)}(l)+\ldots+\eta^{(1)}(k)$. Portanto, para testarmos as hipóteses

$$
\left\{\begin{array}{l}
H_{0}: h^{(1)}(l)=h_{0}^{(1)}(l) \\
H_{1}: h^{(1)}(l) \neq h_{0}^{(1)}(l)
\end{array}\right.
$$

em que $h_{0}^{(1)}(l) \in[0,1]$ utilizamos a seguinte estatística de teste

$$
W^{h}(l)=\frac{\sqrt{n}\left[\hat{h}^{n}(l)-h^{(1)}(l)\right]}{\sqrt{\left[h^{(1)}(l)-h^{(1)^{2}}(l)\right] / \theta^{(1)}(l)}}
$$

que sob $H_{0}$ é dada por

$$
W_{0}^{h}(l)=\frac{\sqrt{n}\left[\hat{h}^{n}(l)-h_{0}^{(1)}(l)\right]}{\sqrt{\left[h^{(1)}(l)-h^{(1)^{2}}(l)\right] / \theta^{(1)}(l)}}
$$

e tem distribuição assintóticamente normal com média 0 e variância 1. No lugar dos parâmetros $h^{(1)}$ e $\theta^{(1)}$, utilizamos os estimadores $\hat{h}^{n}$ e $\hat{\theta}^{n}$, pois tratam-se de estimadores consistentes. Com isso, a estatística de teste utilizada é dada por

$$
W_{0}^{h}(l)=\frac{\sqrt{n}\left[\hat{h}^{n}(l)-h_{0}^{(1)}(l)\right]}{\sqrt{\left[\hat{h}^{n}(l)-\hat{h}^{n^{2}}(l)\right] / \hat{\theta}^{(1)}(l)}}
$$

tem distribuição assintóticamente normal com média 0 e variância 1 . Observe que ao testarmos as hipóteses 5.1, estamos testando se a função intensidade na categoria $l$ é igual a um determinado valor.

Para testarmos hipóteses relacionadas a função intensidade acumulada, por exemplo,

$$
\left\{\begin{array}{l}
H_{0}: H^{(1)}(i)=H_{0}^{(1)}(i) \\
H_{1}: H^{(1)}(i) \neq H_{0}^{(1)}(i)
\end{array}\right.
$$

em que $H_{0}^{(1)}(i) \in \mathbb{R}$, utilizaremos a estatística

$$
W^{H}(l)=\frac{\sqrt{n}\left[\hat{H}^{n}(i)-H^{(1)}(i)\right]}{\sqrt{\sum_{l=1}^{i}\left[h^{(1)}(l)-h^{(1)^{2}}(l)\right] / \theta^{(1)}(l)}}
$$

que sob $H_{0}$ é dada por

$$
W_{0}^{H}(l)=\frac{\sqrt{n}\left[\hat{H}^{n}(i)-H_{0}^{(1)}(i)\right]}{\sqrt{\sum_{l=1}^{i}\left[h^{(1)}(l)-h^{(1)^{2}}(l)\right] / \theta^{(1)}(l)}}
$$


e tem distribuição assintóticamente normal com média 0 e variância 1 . Da mesma forma que discutido anteriormente, substituímos os parâmetros pelos seus respectivos estimadores, pois se tratam de estimadores consistentes. Portanto, a estatística utilizada dada por

$$
W_{0}^{H}(l)=\frac{\sqrt{n}\left[\hat{H}^{n}(i)-H_{0}^{(1)}(i)\right]}{\sqrt{\sum_{l=1}^{i}\left[\hat{h}^{n}(l)-\hat{h}^{n^{2}}(l)\right] / \hat{\theta}^{(1)}(l)}}
$$

tem distribuição assintótica normal com média 0 e variância 1.

Observe que ao testarmos as hipóteses 5.4, estamos testando se a função intensidade acumulada até a categoria $i$, com $i \in\{1, \ldots, k\}$ é igual a um determinado valor.

\subsection{Teste de Hipóteses para Duas amostras}

Agora iremos apresentar a estatística para comparação de duas populações. Consideremos uma amostra aleatória simples de tamanho $n_{1}, X^{(1)}=\left(\left(W_{1}^{(1)}, C_{1}^{(1)}\right), \ldots,\left(W_{n_{1}}^{(1)}, C_{n_{1}}^{(1)}\right)\right)$, e uma amostra aleatória de tamanho $n_{2}, X^{(2)}=\left(\left(W_{1}^{(2)}, C_{1}^{(2)}\right), \ldots,\left(W_{n_{1}}^{(2)}, C_{n_{1}}^{(2)}\right)\right)$, com $X^{(1)}$ e $X^{(2)}$ independentes.

Para comparar as curvas de duas populações iremos introduzir o teste de logrank. Consideremos $l$ categorias, $l=1, \ldots, i$ com $i \in\{1, \ldots, k\}$, que são os possíveis valores que a variável aleatória pode assumir. Utilizando a notação introduzida no Capítulo 3 , temos que $\Delta N^{n_{p}}(l,$. e $V^{n_{p}}(l,),. p=1,2$, denotam o número de observações ou itens em que ocorreu o evento de interesse e o número de observações ou itens sob risco, respectivamente, na categoria $l$. Denotamos por $\Delta N(l,)=.\Delta N^{n_{1}}(l,)+.\Delta N^{n_{2}}(l,$.$) e V(l,)=.V^{n_{1}}(l,)+.V^{n_{2}}(l,$.$) . Os dados em uma$ categoria expecífica $l$ podem ser resumidos conforme a Tabela 5.1.

Tabela 5.1: Número de itens em que ocorreu o evento de interesse e número de itens sob risco na categoria l, por amostra.

\section{Amostras}

\begin{tabular}{cccc} 
Ocorrência do evento de interesse & 1 & 2 & Total \\
\hline Sim & $\Delta N^{n_{1}}(l,)$. & $\Delta N^{n_{2}}(l,)$. & $\Delta N(l,)$. \\
Não & $V^{n_{1}}(l,)-.\Delta N^{n_{1}}(l,)$. & $V^{n_{2}}(l,)-.\Delta N^{n_{2}}(l,)$. & $V(l,)-.\Delta N(l,)$. \\
\hline Total & $V^{n_{1}}(l,)$. & $V^{n_{2}}(l,)$. & $V(l,)$. \\
\hline
\end{tabular}


Sabemos que $\Delta N^{n_{p}}(l,.) / V^{n_{p}}(l,$.$) tem distribuição binomial com número de enasaios V^{n_{p}}(l,$. e probabilidade $h^{(p)}(l), p=1,2$. Assim, obtemos que $\Delta N^{n_{p}}(l,.) / V^{n_{p}}(l,.) ; \Delta N(l,$.$) tem dis-$ tribuição hipergeométrica com parâmetros $V^{n_{p}}(l,),. V(l,$.$) e \Delta N(l,$.$) . Com isso, a esperança e$ a variância da variável $\Delta N^{n_{1}}(l,.) / V^{n_{1}}(l,.) ; \Delta N(l,$.$) são dadas por$

$$
\mathrm{E}^{(1)}(l)=\mathrm{E}\left[\Delta N^{n_{1}}(l, .) / V^{n_{1}}(l, .) ; \Delta N(l, .)\right]=\Delta N(l, .) \frac{V^{n_{1}}(l, .)}{V(l, .)}
$$

$\mathrm{e}$

$$
\operatorname{Var}^{(1)}(l)=\Delta N(l, .) \frac{V^{n_{1}}(l, .) V^{n_{2}}(l, .)}{V^{2}(l, .)} \frac{V(l, .)-\Delta N(l, .)}{V(l, .)-1}
$$

Consideremos o vetor formado pela diferença entre o número de itens observados em que ocorreram o evento de interesse e o número esperado em cada categoria, relacionado a amostra 1 ,

$$
\left\{\Delta N^{n_{1}}(1, .)-E_{1}^{1}, \Delta N^{n_{1}}(2, .)-E_{2}^{1}, \ldots, \Delta N^{n_{1}}(i, .)-E_{i}^{1}\right\}
$$


para $i=1, \ldots, k$, e $Q$ a soma de cada um dos termos deste vetor.

$$
\begin{aligned}
& W=\sum_{l=1}^{i}\left(\Delta N^{n_{1}}(l, .)-E_{l}^{(1)}\right) \\
& =\sum_{l=1}^{i} \Delta N^{n_{1}}(l, .)-\sum_{l=1}^{i} \frac{\Delta N(l, .) V^{n_{1}}(l, .)}{V(l, .)} \\
& =\sum_{m=1}^{n_{1}} \sum_{l=1}^{i} \Delta N_{m}^{(1)}(l, .)-\sum_{p=1}^{2} \sum_{m=1}^{n_{p}} \sum_{l=1}^{i} \Delta N_{m}^{(p)}(l, .) \frac{V^{n_{1}}(l, .)}{V(l, .)} \\
& =\sum_{m=1}^{n_{1}} \sum_{l=1}^{i} \Delta N_{m}^{(1)}(l, .)-\left[\sum_{m=1}^{n_{1}} \sum_{l=1}^{i} \Delta N_{m}^{(1)}(l, .) \frac{V^{n_{1}}(l, .)}{V(l, .)}+\sum_{m=1}^{n_{2}} \sum_{l=1}^{i} \Delta N_{m}^{(2)}(l, .) \frac{V^{n_{1}}(l, .)}{V(l, .)}\right] \\
& =\sum_{m=1}^{n_{1}} \sum_{l=1}^{k}\left[1-\frac{V^{n_{1}}(l, .)}{V(l, .)}\right] \Delta N_{m}^{(1)}(l, .)-\sum_{m=1}^{n_{2}} \sum_{l=1}^{k} \Delta N_{m}^{(2)}(l, .) \frac{V^{n_{1}}(l, .)}{V(l, .)} \\
& =\sum_{m=1}^{n_{1}} \sum_{l=1}^{i} \frac{V^{n_{2}}(l, .)}{V(l, .)} \Delta N_{m}^{(1)}(l, .)-\sum_{m=1}^{n_{2}} \sum_{l=1}^{i} \frac{V^{n_{1}}(l, .)}{V(l, .)} \Delta N_{m}^{(2)}(l, .) \\
& =\sum_{m=1}^{n_{1}} \sum_{l=1}^{i} \frac{V^{n_{2}}(l, .) V^{n_{1}}(l, .)}{V(l, .)}\left\{V^{n_{1}}(l, .)\right\}^{-1}\left[\Delta N_{m}^{(1)}(l, .)-V_{m}^{(1)}(l, .) \Delta H^{(1)}(l)\right] \\
& -\sum_{m=1}^{n_{2}} \sum_{l=1}^{i} \frac{V^{n_{1}}(l, .) V^{n_{2}}(l, .)}{V(l, .)}\left\{V^{n_{2}}(l, .)\right\}^{-1}\left[\Delta N_{m}^{(2)}(l, .)-V_{m}^{(2)}(l, .) \Delta H^{(2)}(l)\right] \\
& =\sum_{m=1}^{n_{1}} \sum_{l=1}^{i} \frac{V^{n_{2}}(l, .)}{V(l, .)} V^{n_{1}}(l, .)\left\{V^{n_{1}}(l, .)\right\}^{-1}\left[\Delta N_{m}^{(1)}(l, .)-\Delta A_{m}^{(1)}(l, .)\right] \\
& -\sum_{m=1}^{n_{2}} \sum_{l=1}^{i} \frac{V^{n_{1}}(l, .) V^{n_{2}}(l, .)}{V(l, .)}\left\{V^{n_{2}}(l, .)\right\}^{-1}\left[\Delta N_{m}^{(2)}(l, .)-\Delta A_{m}^{(1)}(l, .)\right] \\
& =\sum_{l=1}^{i} \frac{V^{n_{2}}(l, .) V^{n_{1}}(l, .)}{V(l, .)}\left[\sum_{m=1}^{n_{1}} \frac{\Delta Y_{m}^{(1)}(l, .)}{V^{n_{1}}(l, .)}-\sum_{m=1}^{n_{2}} \frac{\Delta Y_{m}^{(2)}(l, .)}{V^{n_{2}}(l, .)}\right] \\
& =\sum_{l=1}^{i} \frac{V^{n_{2}}(l, .) V^{n_{1}}(l, .)}{V(l, .)}\left[\frac{\Delta Y^{n_{1}}(l, .)}{V^{n_{1}}(l, .)}-\frac{\Delta Y^{n_{2}}(l, .)}{V^{n_{2}}(l, .)}\right]
\end{aligned}
$$

Esta é derivação de Mantel da estatística de logrank (Fleming \& Harrington (2005)). A seguir, utilizaremos a versão da estatística de logrank proposta por Fleming \& Harrington 
(2005), conforme abaixo,

$$
\begin{aligned}
W & =\sum_{l=1}^{i}\left(\frac{n_{1}+n_{2}}{n_{1} n_{2}}\right)^{1 / 2} \frac{V^{n_{2}}(l, .) V^{n_{1}}(l, .)}{V(l, .)}\left[\frac{\Delta Y^{n_{1}}(l, .)}{V^{n_{1}}(l, .)}-\frac{\Delta Y^{n_{2}}(l, .)}{V^{n_{2}}(l, .)}\right] \\
& =\sum_{l=1}^{i}\left(\frac{n_{1}+n_{2}}{n_{1} n_{2}}\right)^{1 / 2} \frac{V^{n_{2}}(l, .) V^{n_{1}}(l, .)}{V(l, .)}\left[\left(\frac{\Delta N^{n_{1}}(l, .)}{V^{n_{1}}(l, .)}-\Delta H^{(1)}(l)\right)-\left(\frac{\Delta N^{n_{2}}(l, .)}{V^{n_{2}}(l, .)}-\Delta H^{(2)}(l)\right)\right] \\
& =\sum_{l=1}^{i}\left(\frac{n_{1}+n_{2}}{n_{1} n_{2}}\right)^{1 / 2} \frac{V^{n_{2}}(l, .) V^{n_{1}}(l, .)}{V(l, .)}\left[\left(\hat{h}^{n_{1}}(l)-h^{(1)}(l)\right)-\left(\hat{h}^{n_{2}}(l)-h^{(2)}(l)\right)\right]
\end{aligned}
$$

Sob a hipótese nula $H_{0}: H^{n_{1}}=H^{n_{2}}$, dada por

$$
W=\sum_{l=1}^{i}\left(\frac{n_{1}+n_{2}}{n_{1} n_{2}}\right)^{1 / 2} \frac{V^{n_{2}}(l, .) V^{n_{1}}(l, .)}{V(l, .)}\left[\hat{h}^{n_{1}}(l)-\hat{h}^{n_{2}}(l)\right]
$$

e tem distribuição normal com média 0 e variância

$$
\sigma^{2}(i)=\sum_{l=1}^{i}\left(\left[1-h^{(1) *}(l)\right] h^{(1)}(l) G_{l}^{1}+\left[1-h^{(2)}(l)\right] h^{(2)}(l) G_{l}^{2}\right) .
$$

Um estimador para a variância $\sigma^{2}(i)$, conforme proposto por Fleming \& Harrington (2005), é dado por

$$
\hat{\sigma}^{2}(i)=\sum_{l=1}^{i} \frac{U^{n_{1}, n_{2} 2}(l, .)}{V^{n_{1}}(l, .)}\left[1-\hat{h}^{n_{1}}(l)\right] \hat{h}^{n_{1}}(l)+\sum_{l=1}^{i} \frac{U^{n_{1}, n_{2} 2}(l, .)}{V^{n_{2}}(l, .)}\left[1-\hat{h}^{n_{2}}(l)\right] \hat{h}^{n_{2}}(l) .
$$

Observe que com a estatística de teste $W$ obtida, ao testarmos a hipótese $H_{0}: H^{(1)}=H^{(2)}$, estamos testando a igualdade entre as curvas das duas populações. 


\section{Capítulo 6}

\section{Estudo de simulação}

\subsection{Introdução}

Neste capítulo avaliamos numericamente a qualidade das aproximações assintóticas utilizadas nas inferências sobre os parâmetros do modelo de intensidade multiplicativo. Particularmente, o interesse é avaliar a consistências dos estimadores da função intensidade $h^{(p)}$, da função intensidade acumulada $H^{(p)}$ e da distribuição de probabilidade $\zeta^{(p)}$, além de avaliar a qualidade dos testes apresentados nesse trabalho.

As simulações computacionais deste trabalho foram realizadas usando a versão 2.8.0 do software estatístico R. Os resultados numéricos apresentados neste capítulo baseiam-se no modelo de intensidade multiplicativo proposto neste trabalho e na teoria desenvolvida a partir dele, apresentados nos capítulos 3, 4 e 5 .

O modelo proposto pode ser utilizado para analisar dados multinomiais na presença ou não de censura. Foram realizados estudos de simulação para os dois casos.

\subsection{Modelo na ausência de censura}

Inicialmente, consideramos dados multinomiais na ausência de censura. Foram realizadas simulações considerando $k=3,7$ e 10 categorias. Os passos para a simulação são descritos a seguir. 
1. Definimos valores para a probabilidade de ocorrência do evento de interesse em cada uma das categorias, $\zeta^{(p)}(1), \ldots, \zeta^{(p)}(k)$, de forma que a soma da distribuição de probabilidade some 1. Estes valores são apresentados na Tabela 6.1.

2. A partir desses valores, obtemos os valores da função intensidade $h^{(p)}$ e da função intensidade acumulada $H^{(p)}$ utilizando as equações

$$
h^{(p)}(i)=\frac{\zeta^{(p)}(i)}{\zeta^{(p)}(i)+\ldots+\zeta^{(p)}(k)}
$$

e

$$
H^{(p)}(i)=\sum_{l=1}^{i} h^{(p)}(i)
$$

para $i=1, \ldots, k$. Os valores obtidos da função intensidade $h^{(p)}$ e da função intensidade acumulada $H^{(p)}$ também são apresentados na Tabela 6.1 .

Tabela 6.1: Valores da distribuição de probabilidade $\zeta$, da função intensidade $h$ e da função intensidade acumulada H para 3, 7 e 10 categorias.

\begin{tabular}{cccccccccc}
\hline & \multicolumn{3}{c}{3 categorias } & \multicolumn{3}{c}{7 categorias } & \multicolumn{3}{c}{10 categorias } \\
\hline Categoria & $\zeta^{(p)}$ & $h^{(p)}$ & $H^{(p)}$ & $\zeta^{(p)}$ & $h^{(p)}$ & $H^{(p)}$ & $\zeta^{(p)}$ & $h^{(p)}$ & $H^{(p)}$ \\
\hline 1 & 0,2 & 0,200 & 0,200 & 0,16 & 0,160 & 0,160 & 0,10 & 0,100 & 0,100 \\
2 & 0,5 & 0,625 & 0,825 & 0,35 & 0,417 & 0,577 & 0,25 & 0,278 & 0,378 \\
3 & 0,3 & 1,000 & 1,825 & 0,20 & 0,408 & 0,985 & 0,17 & 0,262 & 0,639 \\
4 & & & & 0,06 & 0,207 & 1,192 & 0,09 & 0,188 & 0,827 \\
5 & & & & 0,08 & 0,348 & 1,540 & 0,13 & 0,333 & 1,160 \\
6 & & & & 0,05 & 0,333 & 1,873 & 0,04 & 0,192 & 1,353 \\
7 & & & & 0,03 & 0,300 & 2,173 & 0,03 & 0,205 & 1,557 \\
8 & & & & 0,07 & 1,000 & 3,173 & 0,05 & 0,479 & 2,036 \\
9 & & & & & & & 0,06 & 0,690 & 2,726 \\
10 & & & & & & & 0,077 & 1,000 & 3,726 \\
\hline
\end{tabular}

3. Consideramos amostras de tamanhos $20,50,80,100$ para avaliarmos a qualidade dos estimadores e 20,50, 80, 100, 200, 500 para avaliarmos a qualidade dos testes de hipóteses. 
4. O número de iterações foi fixado em 10000.

5. Para cada iteração geramos uma amostra aleatória da variável multinomial $X$ utilizando a distribuição uniforme com parâmetros 0 e 1.

6. Calculamos os estimadores da função intensidade $h^{(p)}$, da função intensidade acumulada $H^{(p)}$ e da função distribuição de probabilidade $\zeta^{(p)}$ em cada categoria $i, i=1, \ldots, k$, para cada iteração, utilizado as seguintes equações

$$
\begin{gathered}
\hat{h}^{n_{p}}(i)=\frac{\Delta N^{n_{p}}(i, .)}{V^{n_{p}}(i, .)}, \quad \hat{H}^{n_{p}}(i)=\sum_{l=1}^{i} \frac{\Delta N^{n_{p}}(l, .)}{V^{n_{p}}(l, .)} \\
\hat{\zeta}^{n_{p}}(i)=\hat{h}^{n_{p}}(i) \prod_{l=1}^{i-1}\left[1-\hat{h}^{n_{p}}(l)\right] .
\end{gathered}
$$

7. Calculamos os erros quadráticos da função intensidade $h^{(p)}$, da função intensidade acumulada $H^{(p)}$ e da função distribuição de probabilidade $\zeta^{(p)}$ para cada uma das iterações, dados respectivamente, por

$$
\begin{gathered}
e_{h}(i)=\left[\hat{h}^{n_{p}}(i)-h^{(p)}(i)\right]^{2}, \quad e_{H}(i)=\left[\hat{H}^{n_{p}}(i)-H^{(p)}(i)\right]^{2} \\
e_{\zeta}(i)=\left[\hat{\zeta}^{n_{p}}(i)-\zeta^{(p)}(i)\right]^{2}
\end{gathered}
$$

8. Ao final das 10000 iterações, calculamos a média por categoria dos erros quadráticos, os erros quadráticos médios (EQM).

\subsubsection{Avaliação da qualidade dos estimadores}

Nas Tabelas 6.2, 6.3 e 6.4 apresentamos o erros quadráticos médios da função intensidade, da função intensidade acumulada e da distribuição de probabilidade. Observamos que o erro quadrático médio dos estimadores dos parâmetros do modelo de intensidade multiplicativo apresentam uma diferença em relação aos verdadeiros valores a partir da segunda casa decimal. Essa conclusão é obtida no caso de 3 categorias para amostras pequenas e grandes. Já para 7 e 10 categorias, a diferença dos estimadores em relação aos verdadeiros valores chega a quarta casa decimal para amostras grandes. 
Com isso, concluímos que a partir de amostras de tamanho 80 obtemos boas aproximações dos valores dos estimadores dos parâmetros do modelo de intensidade multiplicativo aos valores reais, independente do número de categorias.

Tabela 6.2: Valores dos erros quadráticos médios associados à função intensidade, à função intensidade acumulada e à distribuição de probabilidade para 3 categorias - sem censura.

\begin{tabular}{rrrrrrrrr}
\hline \multicolumn{10}{c}{3 Categorias } & & & \\
Categoria & $n_{p}$ & $E Q M_{h}$ & $E Q M_{H}$ & $E Q M_{\zeta}$ & $n_{p}$ & $E Q M_{h}$ & $E Q M_{H}$ & $E Q M_{\zeta}$ \\
\hline 1 & & 0,00807 & 0,00807 & 0,00807 & & 0,00198 & 0,00198 & 0,00198 \\
2 & 20 & 0,01514 & 0,02308 & 0,01275 & 80 & 0,00365 & 0,00567 & 0,00308 \\
3 & 0 & 0,02290 & 0,01059 & 0 & 0,00567 & 0,00261 \\
\hline 1 & 0,00320 & 0,00320 & 0,00320 & 0,00159 & 0,00159 & 0,00159 \\
2 & 50 & 0,00598 & 0,00926 & 0,00502 & 100 & 0,00290 & 0,00448 & 0,00248 \\
3 & 0 & 0,00926 & 0,00428 & & 0 & 0,00448 & 0,00207 \\
\hline
\end{tabular}


Tabela 6.3: Valores dos erros quadráticos médios associados à função intensidade, à função intensidade acumulada e à distribuição de probabilidade para 7 categorias - sem censura.

\begin{tabular}{|c|c|c|c|c|c|c|c|c|}
\hline \multicolumn{9}{|c|}{7 Categorias } \\
\hline Categoria & $n_{p}$ & $E Q M_{h}$ & $E Q M_{H}$ & $E Q M_{\zeta}$ & $n_{p}$ & $E Q M_{h}$ & $E Q M_{H}$ & $E Q M_{\zeta}$ \\
\hline 1 & & 0,00684 & 0,00684 & 0,00684 & & 0,00167 & 0,00167 & 0,00167 \\
\hline 2 & & 0,01491 & 0,02163 & 0,01158 & & 0,00364 & 0,00523 & 0,00288 \\
\hline 3 & & 0,02726 & 0,04901 & 0,00951 & & 0,00638 & 0,01161 & 0,00232 \\
\hline 4 & 20 & 0,04819 & 0,09578 & 0,00279 & 80 & 0,01010 & 0,02167 & 0,00071 \\
\hline 5 & & 0,08340 & 0,15973 & 0,00317 & & 0,01748 & 0,03854 & 0,00083 \\
\hline 6 & & 0,11977 & 0,20869 & 0,00251 & & 0,03217 & 0,07111 & 0,00070 \\
\hline 7 & & 0,00000 & 0,12129 & 0,00147 & & 0,00000 & 0,06026 & 0,00055 \\
\hline 1 & & 0,00271 & 0,00271 & 0,00271 & & 0,00136 & 0,00136 & 0,00136 \\
\hline 2 & & 0,00590 & 0,00869 & 0,00460 & & 0,00296 & 0,00439 & 0,00230 \\
\hline 3 & & 0,01053 & 0,01945 & 0,00376 & & 0,00505 & 0,00945 & 0,00186 \\
\hline 4 & 50 & 0,01702 & 0,03694 & 0,00115 & 100 & 0,00810 & 0,01760 & 0,00056 \\
\hline 5 & & 0,02949 & 0,06614 & 0,00131 & & 0,01423 & 0,03194 & 0,00065 \\
\hline 6 & & 0,05605 & 0,11829 & 0,00112 & & 0,02476 & 0,05757 & 0,00056 \\
\hline 7 & & 0,00000 & 0,07165 & 0,00074 & & 0,00000 & 0,05335 & 0,00046 \\
\hline
\end{tabular}


Tabela 6.4: Valores dos erros quadráticos médios associados à função intensidade, à função intensidade acumulada e à distribuição de probabilidade para 10 categorias - sem censura.

\begin{tabular}{|c|c|c|c|c|c|c|c|c|}
\hline \multicolumn{9}{|c|}{10 Categorias } \\
\hline Categoria & $n_{p}$ & $E Q M_{h}$ & $E Q M_{H}$ & $E Q M_{\zeta}$ & $n_{p}$ & $E Q M_{h}$ & $E Q M_{H}$ & $E Q M_{\zeta}$ \\
\hline 1 & \multirow{10}{*}{20} & 0,00453 & 0,00453 & 0,00453 & \multirow{10}{*}{80} & 0,00113 & 0,00113 & 0,00113 \\
\hline 2 & & 0,01114 & 0,01584 & 0,00929 & & 0,00275 & 0,00393 & 0,00230 \\
\hline 3 & & 0,01538 & 0,03100 & 0,00704 & & 0,00370 & 0,00784 & 0,00173 \\
\hline 4 & & 0,01711 & 0,04883 & 0,00414 & & 0,00398 & 0,01158 & 0,00104 \\
\hline 5 & & 0,03114 & 0,07927 & 0,00561 & & 0,00724 & 0,01858 & 0,00143 \\
\hline 6 & & 0,03005 & 0,10674 & 0,00189 & & 0,00652 & 0,02496 & 0,00048 \\
\hline 7 & & 0,03569 & 0,12934 & 0,00161 & & 0,00772 & 0,03227 & 0,00041 \\
\hline 8 & & 0,06569 & 0,17366 & 0,00236 & & 0,01367 & 0,04583 & 0,00058 \\
\hline 9 & & 0,10688 & 0,22260 & 0,00265 & & 0,02476 & 0,06916 & 0,00071 \\
\hline 10 & & 0,00000 & 0,11938 & 0,00225 & & 0,00000 & 0,06725 & 0,00085 \\
\hline 1 & \multirow{10}{*}{50} & 0,00179 & 0,00179 & 0,00179 & \multirow{10}{*}{100} & 0,00090 & 0,00090 & 0,00090 \\
\hline 2 & & 0,00439 & 0,00623 & 0,00366 & & 0,00225 & 0,00311 & 0,00190 \\
\hline 3 & & 0,00608 & 0,01247 & 0,00281 & & 0,00297 & 0,00620 & 0,00139 \\
\hline 4 & & 0,00657 & 0,01895 & 0,00165 & & 0,00324 & 0,00953 & 0,00082 \\
\hline 5 & & 0,01191 & 0,03056 & 0,00231 & & 0,00581 & 0,01533 & 0,00115 \\
\hline 6 & & 0,01059 & 0,04127 & 0,00077 & & 0,00507 & 0,02017 & 0,00038 \\
\hline 7 & & 0,01287 & 0,05391 & 0,00065 & & 0,00583 & 0,02600 & 0,00031 \\
\hline 8 & & 0,02379 & 0,07825 & 0,00095 & & 0,01082 & 0,03668 & 0,00047 \\
\hline 9 & & 0,04216 & 0,11571 & 0,00114 & & 0,01907 & 0,05593 & 0,00056 \\
\hline 10 & & 0,00000 & 0,09659 & 0,00128 & & 0,00000 & 0,05532 & 0,00070 \\
\hline
\end{tabular}




\subsubsection{Teste de hipótese}

Nesta seção iremos avaliar a qualidade dos desempenhos dos testes de hipóteses, para uma e duas populações.

\section{Uma população}

O desempenho do teste para uma população é avaliado em função da proporção de vezes em que não rejeitamos a hipótese nula, dado que simulamos sob a hipótese $H_{0}: H^{(p)}(i)=H_{0}^{(p)}(i)$, ao nível de confiança de $95 \%$.

Para realizar este estudo de simulação, devemos seguir as indicações apresentadas na seção anterior até o sexto passo. A partir daí, o procedimento é o seguinte:

7. Utilizando os estimadores da função intensidade acumulada e da distribuição de probabilidade, $\hat{H}^{n_{p}}$ e $\hat{\zeta}^{n_{p}}$, calculamos as estatísticas do teste para uma população para cada iteração. A estatística é dada por

$$
W_{0}^{H}=\frac{\sqrt{n_{p}}\left[\hat{H}^{n_{p}}(i)-H_{0}^{\left(n_{p}\right)}(i)\right]}{\sqrt{\sum_{l=1}^{i}\left[1-\hat{h}^{\left(n_{p}\right)}(l)\right] \hat{h}^{\left(n_{p}\right)}(l) / \hat{\theta}_{l}^{\left(n_{p}\right)}}},
$$

em que $\hat{\theta}_{l}^{\left(n_{p}\right)}=\hat{\zeta}^{n_{p}}(l)+\ldots+\hat{\zeta}^{n_{p}}(k)$. A estatística $W_{0}^{H}$ tem distribuição assintótica normal com média 0 e variância 1 .

8. Com nível de significância de 5\%, calculamos a porporção de vezes em que não rejeitamos a hipótese $H_{0}$.

Na Tabela 6.11 temos os resultados dos testes de hipótese para 3, 7 e 10 categorias e para diferentes tamanhos de amostras $\left(n_{p}\right)$. Observamos que os níveis de confianças empíricos dos testes aproximam-se do nível de confiância adotado de $95 \%$ com o aumento do tamanho amostral, conforme esperado. 
Tabela 6.5: Teste de hipótese para a função acumualada até a terceira, sétima e décima categoria - uma amostra.

\begin{tabular}{lccc}
\hline & \multicolumn{3}{c}{ Categorias } \\
Amostra & 3 & 7 & 10 \\
\hline 20 & 92,85 & 90,49 & 92,36 \\
50 & 94,32 & 94,11 & 94,40 \\
80 & 94,66 & 94,69 & 94,27 \\
100 & 94,69 & 94,04 & 94,53 \\
200 & 94,80 & 94,65 & 94,65 \\
500 & 95,01 & 94,79 & 95,25 \\
\hline
\end{tabular}

\section{Duas populações}

Realizamos testes de comparação entre o comportamento das funções intensidades acumuladas das duas populações até a $3^{\circ}, 7^{\circ}$ e $10^{\circ}$ categorias. O desempenho do teste para comparação de duas populações é avaliado em função da proporção de vezes em que não rejeitamos a hipótese nula, dado que simulamos sob a hipótese $H_{0}: H^{(p)}=H_{0}^{(p)}$, ao nível de confiança de $95 \%$.

Os passos para este estudo de simulação são apresentados a seguir.

1. Definimos valores para a probabilidade de ocorrência do evento de interesse em cada uma das categorias para as duas populações, $\zeta(1), \ldots, \zeta(k)$.

2. Consideramos amostras de tamanhos 20,50,80,100,200 e 500 para as duas populações. Neste estudo de simulação, comparamos apenas amostras de tamanhos iguais.

3. O número de iterações foi fixado em 10000.

4. Para cada iteração geramos duas amostras aleatórias utilizando a distribuição uniforme com parâmetros 0 e 1 e a distribuição de probabilidade.

5. Calculamos os estimadores das funções intensidades $h^{(1)}$ e $h^{(2)}$, da função intensidade acumulada $H^{(1)}$ e $H^{(2)}$ por categoria para cada uma das iterações utilizando as seguintes 
equações

$$
\hat{h}^{n_{p}}(i)=\frac{\Delta N^{n_{p}}(i, .)}{V^{n_{p}}(i, .)}, \quad \hat{H}^{n_{p}}(i)=\sum_{l=1}^{i} \frac{\Delta N^{n_{p}}(l, .)}{V^{n_{p}}(l, .)}
$$

para $p=1,2$.

6. Utilizando os estimadores calculamos o valor da estatística de teste para comparação entre as duas populações, dada por

$$
W=\frac{\sum_{l=1}^{i}\left(\frac{n_{1}+n_{2}}{n_{1} n_{2}}\right)^{1 / 2} \frac{V^{n_{2}}(l, .) V^{n_{1}}(l, .)}{V(l, .)}\left[\hat{h}^{n_{1}}(l)-\hat{h}^{n_{2}}(l)\right]}{\sqrt{\hat{\sigma}^{2}(i)}}
$$

em que $V(l,)=.V^{n_{2}}(l,)+.V^{n_{2}}(l,$.$) e$

$$
\hat{\sigma}^{2}(i)=\sum_{l=1}^{i} \frac{U^{n_{1}, n_{2} 2}(l, .)}{V^{n_{1}}(l, .)}\left[1-\hat{h}^{n_{1} *}(l)\right] \hat{h}^{n_{1} *}(l)+\sum_{l=1}^{i} \frac{U^{n_{1}, n_{2} 2}(l, .)}{V^{n_{2}}(l, .)}\left[1-\hat{h}^{n_{2} *}(l)\right] \hat{h}^{n_{2} *}(l) .
$$

A estatística $W$ tem distribuição assintótica normal com média 0 e variância 1.

7. Com nível de significância de 5\%, calculamos a porporção de vezes em que não rejeitamos a hipótese $H_{0}$.

Na Tabela 6.12 temos os resultados dos testes de hipótese para 3, 7 e 10 categorias e para diferentes tamanhos de amostras $\left(n_{1}=n_{2}\right)$. Observa-se que os níveis de confianças empíricos dos testes aproximam-se do nível de confiância adotado de $95 \%$ com o aumento do tamanho amostral, conforme esperado. 
Tabela 6.6: Teste de hipótese para a comparação entre duas populações até a terceira, sétima e décima categoria - duas amostras.

\begin{tabular}{rrrr}
\hline & \multicolumn{3}{c}{ Categorias } \\
$n_{1}=n_{2}$ & 3 & 7 & 10 \\
\hline 20 & 92,79 & 90,49 & 92,36 \\
50 & 94,33 & 95,11 & 95,4 \\
80 & 95,13 & 94,69 & 94,27 \\
100 & 94,85 & 94,04 & 94,53 \\
200 & 94,88 & 94,65 & 94,65 \\
500 & 94,89 & 94,79 & 95,25 \\
\hline
\end{tabular}

\subsection{Análise na presença de censura}

Nesta seção apresentamos o estudo de simulação para dados multinomiais na presença de censura. Os cálculos dos estimadores e as estatística de testes são iguais aos efetuados na análise sem censura. Portanto, ao descrever os passos para a realização do estudo de simualação na presença de censura estaremos sendo repetitivos em algumas descrições.

1. Definimos valores para a probabilidade de ocorrência do evento de interesse $\zeta^{(p)}(1), \ldots, \zeta^{(p)}(k)$

e para a probabilidade de ocorrência do evento de censura $\eta^{(p)}(1)-\zeta^{(p)}(1), \ldots, \eta^{(p)}(k)-$ $\zeta^{(p)}(k)$, em cada uma das categorias. Estes valores são apresentados na Tabela 6.7. Definimos também valores para a probabilidade do item "sobreviver" até o fim do estudo $\eta^{(p)}(k+1): \eta(4)=0,8262, \eta(8)=0,7697$ e $\eta(11)=0,4909$.

2. A partir desses valores, obtemos os valores verdadeiros da função intensidade $h^{(p)}$ e da função intensidade acumulada $H^{(p)}$ para cada categoria utilizando as equações

$$
h^{(p)}(i)=\frac{\zeta^{(p)}(i)}{\zeta^{(p)}(i)+\ldots+\zeta^{(p)}(k)}
$$

e

$$
H^{(p)}(i)=\sum_{l=1}^{i} h^{(p)}(i)
$$


Tabela 6.7: Valores da distribuição de probabilidade $\zeta$, da probabilidade de censura, $\eta-\zeta$, da função intensidade $h$ e da função intensidade acumulada H para 3, 7 e 10 categorias - com censura.

\begin{tabular}{|c|c|c|c|c|c|c|c|c|c|c|c|c|}
\hline & \multicolumn{4}{|c|}{3 categorias } & \multicolumn{4}{|c|}{7 categorias } & \multicolumn{4}{|c|}{10 categorias } \\
\hline & $\zeta^{(p)}$ & $\eta^{(p)}-\zeta^{(p)}$ & $h^{(p)}$ & $H^{(p)}$ & $\zeta^{(p)}$ & $\eta^{(p)}-\zeta^{(p)}$ & $h^{(p)}$ & $H^{(p)}$ & $\zeta^{(p)}$ & $\eta^{(p)}-\zeta^{(p)}$ & $h^{(p)}$ & $H^{(p)}$ \\
\hline 1 & 0,05 & 0,0020 & 0,05 & 0,05 & 0,05 & 0,0020 & 0,05 & 0,05 & 0,05 & 0,0020 & 0,05 & 0,05 \\
\hline 2 & 0,090 & 0,0018 & 0,0949 & 0,1449 & 0,09 & 0,0018 & 0,0949 & 0,1449 & 0,09 & 0,0018 & 0,0949 & 0,1449 \\
\hline 3 & 0,025 & 0,0050 & 0,0292 & 0,1741 & 0,025 & 0,0050 & 0,0292 & 0,1741 & 0,025 & 0,0050 & 0,0292 & 0,1741 \\
\hline 4 & & & & & 0,02 & 0,0040 & 0,0242 & 0,1983 & 0,02 & 0,0040 & 0,0242 & 0,1983 \\
\hline 5 & & & & & 0,01 & 0,0030 & 0,0125 & 0,2108 & 0,01 & 0,0030 & 0,0125 & 0,2108 \\
\hline 6 & & & & & 0,006 & 0,0045 & 0,0076 & 0,2184 & 0,006 & 0,0045 & 0,0076 & 0,2184 \\
\hline 7 & & & & & 0,005 & 0,0040 & 0,0064 & 0,2248 & 0,005 & 0,0040 & 0,0064 & 0,2248 \\
\hline 8 & & & & & & & & & 0,0034 & 0,1100 & 0,0044 & 0,2293 \\
\hline 9 & & & & & & & & & 0,0034 & 0,1100 & 0,0052 & 0,2344 \\
\hline 10 & & & & & & & & & 0,002 & 0,0500 & 0,2381 & 0,2381 \\
\hline
\end{tabular}

para $i=1, \ldots, k$. Os valores obtidos da função intensidade $h^{(p)}$ e da função intensidade acumulada $H^{(p)}$ também são apresentados na Tabela 6.7 .

3. Consideramos amostras de tamanhos 20,50,80, 100 para avaliarmos a qualidade dos estimadores e 20,50,80,100,200,500 para avaliarmos a qualidade dos testes de hipóteses.

4. O número de iterações foi fixado em 10000.

5. Para cada iteração geramos uma amostra aleatória da variável multinomial $X$ utilizando a distribuição uniforme com parâmetros 0 e 1 .

6. Calculamos os estimadores da função intensidade $h^{(p)}$, da função intensidade acumulada $H^{(p)}$, da função distribuição de probabilidade $\zeta^{(p)}$ e de $\eta^{(p)}-\zeta^{(p)}$.

7. Calculamos o erro quadrático desses parâmetros para cada uma das iterações.

8. Ao final das 10000 iterações, calculamos a média por categoria dos erros quadráticos, obtendo os erros quadráticos médios (EQM). 


\subsubsection{Avaliação da qualidade dos estimadores}

Nas Tabelas 6.8, 6.9 e 6.10 apresentamos o erros quadráticos médios relacionados aos parâmetros do modelo de intensidade multiplicativo. Observamos que o erro quadrático médio destes parâmetros apresentam uma diferença em relação aos valores assumidos a partir da segunda casa decimal. Quando aumentamos o tamanho da amostra de 20 para 80, essa diferença chega à quinta casa decimal. Com isso, concluímos que a partir de amostras de tamanho 80 obtemos boas aproximações dos valores dos estimadores dos parâmetros do modelo de intensidade multiplicativo aos valores assumidos e, assim, verificamos a consistência dos estimadores.

Tabela 6.8: Valores dos erros quadráticos médios associados à função intensidade, à função intensidade acumulada e à distribuição de probabilidade para 3 categorias - com censura.

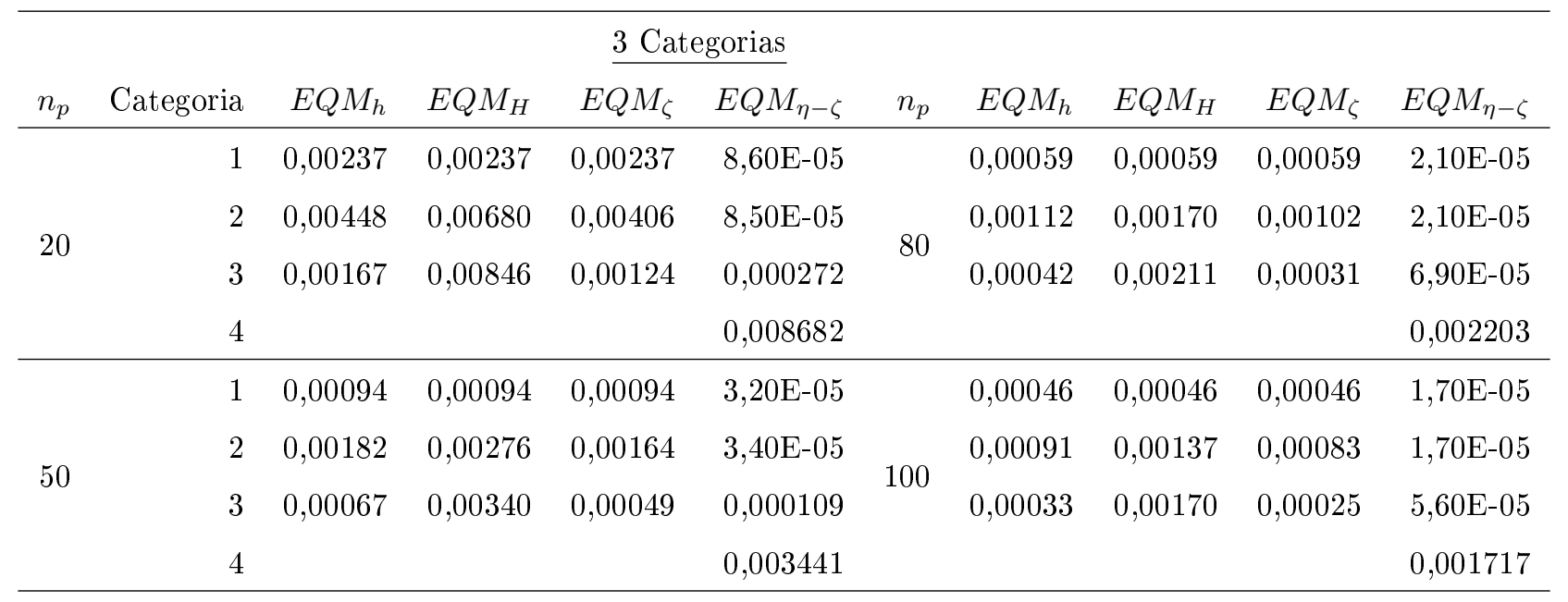


Tabela 6.9: Valores dos erros quadráticos médios associados à função intensidade, à função intensidade acumulada e à distribuição de probabilidade para 7 categorias - com censura.

\begin{tabular}{|c|c|c|c|c|c|c|c|c|c|c|}
\hline \multirow[b]{2}{*}{$n_{p}$} & \multirow[b]{2}{*}{ Categoria } & \multirow[b]{2}{*}{$E Q M_{h}$} & \multicolumn{3}{|c|}{7 Categorias } & \multirow[b]{2}{*}{$n_{p}$} & \multirow[b]{2}{*}{$E Q M_{h}$} & \multirow[b]{2}{*}{$E Q M_{H}$} & \multirow[b]{2}{*}{$E Q M_{\zeta}$} & \multirow[b]{2}{*}{$E Q M_{\eta-\zeta}$} \\
\hline & & & $E Q M_{H}$ & $E Q M_{\zeta}$ & $E Q M_{\eta-\zeta}$ & & & & & \\
\hline \multirow{8}{*}{20} & 1 & 0,0024 & 0,0024 & 0,0024 & $8,10 \mathrm{E}-05$ & \multirow{8}{*}{80} & 0,0006 & 0,0006 & 0,0006 & $2,00 \mathrm{E}-05$ \\
\hline & 2 & 0,0045 & 0,0070 & 0,0041 & $7,80 \mathrm{E}-05$ & & 0,0011 & 0,0017 & 0,0010 & $2,00 \mathrm{E}-05$ \\
\hline & 3 & 0,0017 & 0,0087 & 0,0012 & 0,00027 & & 0,0004 & 0,0022 & 0,0003 & $6,60 \mathrm{E}-05$ \\
\hline & 4 & 0,0015 & 0,0102 & 0,0010 & 0,00025 & & 0,0004 & 0,0025 & 0,0003 & $5,90 \mathrm{E}-05$ \\
\hline & 5 & 0,0008 & 0,0110 & 0,0005 & 0,00018 & & 0,0002 & 0,0027 & 0,0001 & $4,60 \mathrm{E}-05$ \\
\hline & 6 & 0,0005 & 0,0115 & 0,0003 & 0,00030 & & 0,0001 & 0,0028 & 0,0001 & $6,80 \mathrm{E}-05$ \\
\hline & 7 & 0,0004 & 0,0119 & 0,0003 & 0,00024 & & 0,0001 & 0,0029 & 0,0001 & $6,10 \mathrm{E}-05$ \\
\hline & 8 & & & & 0,0113 & & & & & 0,0029 \\
\hline \multirow{8}{*}{50} & 1 & 0,0009 & 0,0009 & 0,0009 & $3,20 \mathrm{E}-05$ & \multirow{8}{*}{100} & 0,0005 & 0,0005 & 0,0005 & $1,60 \mathrm{E}-05$ \\
\hline & 2 & 0,0018 & 0,0027 & 0,0016 & $3,40 \mathrm{E}-05$ & & 0,0009 & 0,0014 & 0,0008 & $1,70 \mathrm{E}-05$ \\
\hline & 3 & 0,0007 & 0,0034 & 0,0005 & 0,000105 & & 0,0003 & 0,0017 & 0,0002 & $5,50 \mathrm{E}-05$ \\
\hline & 4 & 0,0006 & 0,0039 & 0,0004 & $9,10 \mathrm{E}-05$ & & 0,0003 & 0,0020 & 0,0002 & $4,50 \mathrm{E}-05$ \\
\hline & 5 & 0,0003 & 0,0042 & 0,0002 & $6,70 \mathrm{E}-05$ & & 0,0002 & 0,0022 & 0,0001 & $3,50 \mathrm{E}-05$ \\
\hline & 6 & 0,0002 & 0,0044 & 0,0001 & 0,00011 & & $9,40 \mathrm{E}-05$ & 0,002291 & $6,10 \mathrm{E}-05$ & $5,60 \mathrm{E}-05$ \\
\hline & 7 & 0,0002 & 0,0046 & 0,0001 & 0,00010 & & $8,20 \mathrm{E}-05$ & 0,002382 & $5,20 \mathrm{E}-05$ & $5,00 \mathrm{E}-05$ \\
\hline & 8 & & & & 0,0044 & & & & & 0,0022 \\
\hline
\end{tabular}


Tabela 6.10: Valores dos erros quadráticos médios associados à função intensidade, à função intensidade acumulada e à distribuição de probabilidade para 10 categorias - com censura.

\begin{tabular}{|c|c|c|c|c|c|c|c|c|c|c|}
\hline \multirow[b]{2}{*}{$n_{p}$} & \multirow[b]{2}{*}{ Categoria } & \multirow[b]{2}{*}{$E Q M_{h}$} & \multirow[b]{2}{*}{$E Q M_{H}$} & \multicolumn{2}{|c|}{10 Categorias } & \multirow[b]{2}{*}{$n_{p}$} & \multirow[b]{2}{*}{$E Q M_{h}$} & \multirow[b]{2}{*}{$E Q M_{H}$} & \multirow[b]{2}{*}{$E Q M_{\zeta}$} & \multirow[b]{2}{*}{$E Q M_{\eta-\zeta}$} \\
\hline & & & & $E Q M_{\zeta}$ & $E Q M_{\eta-\zeta}$ & & & & & \\
\hline \multirow{11}{*}{20} & 1 & 0,00236 & 0,00236 & 0,00236 & $8,50 \mathrm{E}-05$ & \multirow{11}{*}{80} & 0,00059 & 0,00059 & 0,00059 & $2,00 \mathrm{E}-05$ \\
\hline & 2 & 0,00453 & 0,00682 & 0,00411 & 7,10E-05 & & 0,00112 & 0,00170 & 0,00102 & $2,00 \mathrm{E}-05$ \\
\hline & 3 & 0,00169 & 0,00849 & 0,00125 & 0,00026 & & 0,00041 & 0,00209 & 0,00030 & $6,70 \mathrm{E}-05$ \\
\hline & 4 & 0,00146 & 0,01001 & 0,00101 & 0,00022 & & 0,00035 & 0,00244 & 0,00025 & $5,80 \mathrm{E}-05$ \\
\hline & 5 & 0,00077 & 0,01084 & 0,00050 & 0,00017 & & 0,00019 & 0,00261 & 0,00012 & $4,30 \mathrm{E}-05$ \\
\hline & 6 & 0,00048 & 0,01133 & 0,00030 & 0,00029 & & 0,00012 & 0,00272 & $7,70 \mathrm{E}-05$ & $7,00 \mathrm{E}-05$ \\
\hline & 7 & 0,00041 & 0,01175 & 0,00026 & 0,00025 & & 0,00010 & 0,00284 & $6,50 \mathrm{E}-05$ & $6,30 \mathrm{E}-05$ \\
\hline & 8 & 0,00029 & 0,01197 & 0,00018 & 0,00630 & & $7,00 \mathrm{E}-05$ & 0,00292 & $4,40 \mathrm{E}-05$ & 0,00156 \\
\hline & 9 & 0,00038 & 0,01234 & 0,00024 & 0,00633 & & $9,20 \mathrm{E}-05$ & 0,00302 & $5,70 \mathrm{E}-05$ & 0,00155 \\
\hline & 10 & 0,00029 & 0,01262 & 0,00018 & 0,00305 & & $7,00 \mathrm{E}-05$ & 0,00308 & $4,40 \mathrm{E}-05$ & 0,00077 \\
\hline & 11 & & & & 0,01604 & & & & & 0,00401 \\
\hline \multirow{11}{*}{50} & 1 & 0,00093 & 0,00093 & 0,00093 & $3,10 \mathrm{E}-05$ & \multirow{11}{*}{100} & 0,00047 & 0,00047 & 0,00047 & $1,50 \mathrm{E}-05$ \\
\hline & 2 & 0,00179 & 0,00271 & 0,00162 & $3,10 \mathrm{E}-05$ & & 0,00091 & 0,00140 & 0,00083 & $1,60 \mathrm{E}-05$ \\
\hline & 3 & 0,00066 & 0,00338 & 0,00049 & 0,00011 & & 0,00033 & 0,00174 & 0,00025 & $5,40 \mathrm{E}-05$ \\
\hline & 4 & 0,00056 & 0,00396 & 0,00039 & $9,10 \mathrm{E}-05$ & & 0,00029 & 0,00203 & 0,00020 & $4,40 \mathrm{E}-05$ \\
\hline & 5 & 0,00031 & 0,00429 & 0,00020 & $7,30 \mathrm{E}-05$ & & 0,00016 & 0,00219 & 0,00010 & $3,50 \mathrm{E}-05$ \\
\hline & 6 & 0,00019 & 0,00451 & 0,00012 & 0,00011 & & $9,60 \mathrm{E}-05$ & 0,00227 & $6,20 \mathrm{E}-05$ & $5,40 \mathrm{E}-05$ \\
\hline & 7 & 0,00016 & 0,00467 & $1,00 \mathrm{E}-04$ & $1,00 \mathrm{E}-04$ & & $8,00 \mathrm{E}-05$ & 0,00234 & $5,10 \mathrm{E}-05$ & $4,70 \mathrm{E}-05$ \\
\hline & 8 & 0,00011 & 0,00477 & $6,90 \mathrm{E}-05$ & 0,00246 & & $5,70 \mathrm{E}-05$ & 0,00241 & $3,60 \mathrm{E}-05$ & 0,00123 \\
\hline & 9 & 0,00015 & 0,00492 & $9,20 \mathrm{E}-05$ & 0,00244 & & $7,20 \mathrm{E}-05$ & 0,00248 & $4,50 \mathrm{E}-05$ & 0,00123 \\
\hline & 10 & 0,00012 & 0,00502 & $7,70 \mathrm{E}-05$ & 0,00123 & & $5,70 \mathrm{E}-05$ & 0,00252 & $3,60 \mathrm{E}-05$ & 0,00062 \\
\hline & 11 & & & & 0,00630 & & & & & 0,00315 \\
\hline
\end{tabular}




\subsubsection{Teste de hipótese}

Nesta seção iremos avaliar a qualidade dos desempenhos dos testes de hipóteses, para uma e duas populações.

\section{Uma população}

Da mesmas forma que no caso sem censura, o desempenho do teste para comparação de duas populações é avaliado em função da proporção de vezes em que não rejeitamos a hipótese nula, dado que simulamos sob a hipótese $H_{0}: H^{(p)}=H_{0}^{(p)}$, ao nível de confiança de $95 \%$.

Para realizar este estudo de simulação, devemos seguir as indicações apresentadas na seção anterior até o sexto passo. A partir daí, o procedimento é o seguinte.

7. Utilizando os estimadores $\hat{H}^{n_{p}}$ e $\hat{\eta}^{n_{p}}$ calculamos as estatísticas do teste para uma população para cada iteração. A estatística é dada por

$$
W_{0}^{H}=\frac{\sqrt{n_{p}}\left[\hat{H}^{n_{p}}(i)-H_{0}^{\left(n_{p}\right)}(i)\right]}{\sqrt{\sum_{l=1}^{i}\left[1-\hat{h}^{\left(n_{p}\right)}(l)\right] \hat{h}^{\left(n_{p}\right)}}(l) / \hat{\theta}_{l}^{\left(n_{p}\right)}},
$$

em que $\hat{\theta}^{\left(n_{p}\right)}(l)=\hat{\eta}^{n_{p}}(l)+\ldots+\hat{\eta}^{n_{p}}(k+1)$. A estatística $W_{0}^{H}$ tem distribuição normal com média 0 e variância 1 .

8. Com nível de significância de 5\%, calculamos a porporção de vezes em que não rejeitamos a hipótese $H_{0}$.

Na Tabela 6.11 temos os resultados dos testes de hipótese para 3, 7 e 10 categorias e para diferentes tamanhos de amostras $\left(n_{p}\right)$. Observa-se que os níveis de confianças empíricos dos testes aproximam-se do nível de confiância adotado de 95\% com o aumento do tamanho amostral, conforme esperado. 
Tabela 6.11: Teste de hipótese para a função acumualada até a terceira, sétima e décima categoria - uma amostra.

\begin{tabular}{cccc}
\hline & \multicolumn{3}{c}{ Categorias } \\
$n_{p}$ & 3 & 7 & 10 \\
\hline 20 & 92,85 & 90,49 & 92,36 \\
50 & 94,32 & 94,11 & 94,40 \\
80 & 94,66 & 94,69 & 94,27 \\
100 & 94,69 & 94,04 & 94,53 \\
200 & 94,80 & 94,65 & 94,65 \\
500 & 95,01 & 94,79 & 95,25 \\
\hline
\end{tabular}

\section{Duas populações}

Realizamos testes de comparação entre o comportamento das funções intensidades acumuladas das duas populações até a $3^{\circ}, 7^{\circ}$ e $10^{\circ}$ categorias. Os passos para este estudo de simulação são apresentados a seguir:

1. Assumimos valores para a probabilidade de ocorrência do evento de interesse em cada uma das categorias, para a probabilidade de ocorrência dos eventos de censura e para a probabilidade de um item "sobreviver" até o fim do estudo. Os valores são os mesmos assumidos para a avaliação do estimadores.

2. Consideramos amostras de tamanhos 20,50,80,100,200 e 500 para as duas populações. Neste estudo de simulação, comparamos apenas amostras de tamanhos iguais.

3. O número de iterações foi fixado em 10000.

4. Para cada iteração geramos duas amostras aleatórias utilizando a distribuição uniforme com parâmetros 0 e 1 .

5. Calculamos os estimadores das funções intensidades $h^{(1)}$ e $h^{(2)}$, da função intensidade acumulada $H^{(1)}$ e $H^{(2)}$ por categoria para cada uma das iterações utilizando as seguintes 
equações

$$
\hat{h}^{n_{p}}(i)=\frac{\Delta N^{n_{p}}(i, .)}{V^{n_{p}}(i, .)}, \quad \hat{H}^{n_{p}}(i)=\sum_{l=1}^{i} \frac{\Delta N^{n_{p}}(l, .)}{V^{n_{p}}(l, .)}
$$

para $p=1,2$.

6. Utilizando os estimadores calculamos o valor da estatística de teste para comparação entre as duas populações, dada por

$$
W=\frac{\sum_{l=1}^{i}\left(\frac{n_{1}+n_{2}}{n_{1} n_{2}}\right)^{1 / 2} \frac{V^{n_{2}}(l, .) V^{n_{1}}(l, .)}{V(l, .)}\left[\hat{h}^{n_{1}}(l)-\hat{h}^{n_{2}}(l)\right]}{\sqrt{\hat{\sigma}^{2}(i)}}
$$

em que $V(l,)=.V^{n_{2}}(l,)+.V^{n_{2}}(l,$.$) e$

$$
\hat{\sigma}^{2}(i)=\sum_{l=1}^{i} \frac{U^{n_{1}, n_{2} 2}(l, .)}{V^{n_{1}}(l, .)}\left[1-\hat{h}^{n_{1}}(l)\right] \hat{h}^{n_{1}}(l)+\sum_{l=1}^{i} \frac{U^{n_{1}, n_{2} 2}(l, .)}{V^{n_{2}}(l, .)}\left[1-\hat{h}^{n_{2}}(l)\right] \hat{h}^{n_{2}}(l) .
$$

A estatística $W$ tem distribuição assintótica normal com média 0 e variância.

7. Com nível de significância de 5\%, calculamos a porporção de vezes em que não rejeitamos a hipótese $H_{0}$.

Na Tabela 6.12 temos os resultados dos testes de hipótese para 3, 7 e 10 categorias e para diferentes tamanhos de amostras $\left(n_{1}=n_{2}\right)$. Observa-se que os níveis de confianças empíricos dos testes aproximam-se do nível de confiância adotado de $95 \%$ com o aumento do tamanho amostral, conforme esperado. 
Tabela 6.12: Teste de hipótese para a comparação entre duas populações até a terceira, sétima e décima categoria - duas amostras.

\begin{tabular}{rrrr}
\hline & \multicolumn{3}{c}{ Categorias } \\
$n_{1}=n_{2}$ & 3 & 7 & 10 \\
\hline 20 & 92,79 & 90,49 & 92,36 \\
50 & 94,33 & 95,11 & 95,4 \\
80 & 95,13 & 94,69 & 94,27 \\
100 & 94,85 & 94,04 & 94,53 \\
200 & 94,88 & 94,65 & 94,65 \\
500 & 94,89 & 94,79 & 95,25 \\
\hline
\end{tabular}

\subsection{Conclusão}

Os resultados obtidos indicam que os estimadores dos parâmetros do modelo de intensidade mutiplicativo têm vício desprezível comprovando a consistência dos estimadores. Notamos também que os testes de hipóteses para uma e duas populações forneceram taxas de não rejeição da hipótese nula bem próximas ao nível de confiança de $95 \%$. 


\section{Capítulo 7}

\section{Aplicação}

\subsection{Introdução}

Consideremos o conjunto de dados de alunos de graduação da USP (Fonte: JúpiterWeb) descrito na Seção 1.1. O interesse neste capítulo é analisar o tempo de evasão (em semestres) desses alunos. Para isso associamos a cada aluno uma variável aleatória multinomial que determina o número de semestres até a evasão, caso o aluno tenha evadido, ou o número de semestres até a ocorrência de algum dos eventos de censura, migração, falecimento ou conclusão, e analisamos seu comportamento ao longo do tempo e sua relação com fatores determinantes, como sexo, área e período do curso.

Na primeira seção foram realizadas análises descritivas. Na segunda seção os dados foram analisados utilizando o modelo de intensidade multiplicativo e a teoria de testes de hipóteses desenvolvidos nessa dissertação e apresentados nos Capítulos 3 e 5, respectivamente.

\subsection{Análise Descritiva da Evasão dos Alunos de Graduação da USP}

Consideremos apenas os alunos ingressantes por vestibular entre 2000 e 2004. Conforme visto na Seção 1.1, os alunos foram divididos em 5 classes, segundo a última ocorrência observada no sistema Júpter ao fim do estudo, que se deu em dezembro de 2008. As 5 últimas ocorrências 
são evasão, conclusão, falecimento, migração e em andamento. Na Tabela 7.1 temos o número de alunos que se econtravam em cada uma dessas categorias ao fim do segundo semestre de 2008.

Tabela 7.1: Quantidade de alunos em cada uma das classes de última ocorrência (em andamento, conclusão, evasão, falecimento, migração) por ano.

\begin{tabular}{lrrrrrr}
\hline & \multicolumn{5}{c}{ Ano de ingresso } & \\
\cline { 3 - 6 } Última ocorrência & 2000 & 2001 & 2002 & 2003 & 2004 & Total \\
\hline Em andamento & 255 & 422 & 921 & 1900 & 3932 & 7430 \\
Conclusão & 5148 & 5445 & 5465 & 5055 & 3343 & 24456 \\
Evasão & 1481 & 1248 & 1278 & 1179 & 1102 & 6288 \\
Falecimento & 12 & 7 & 11 & 9 & 5 & 44 \\
Migração & 311 & 336 & 318 & 381 & 388 & 1734 \\
\hline Nro ingressantes & 7207 & 7458 & 7993 & 8524 & 8770 & 39952 \\
\hline
\end{tabular}

Podemos observar que, dos alunos ingressantes em 2000, 5148 concluíram, 1481 evadiram, 12 faleceram, 311 migraram e 255 estavam em andamento, até o fim do segundo semestre de 2008.

O evento de interesse dentre as últimas ocorrências é a evasão e um dos objetivos é estudar seu comportamento ao longo do tempo. Para isso, observe que um aluno ingressante em 2004 teve menos semestres para evadir do que um aluno ingressante em 2000, 2001, 2002 ou 2003. Desta forma, para realizarmos uma análise comparativa da evasão ao longo dos anos devemos observar o comportamento dos alunos apenas até o décimo semestre. Portanto, não é correto utilizarmos os dados como apresentados na Tabela 7.1 para verificarmos o comportamento da evasão ao longo do tempo. Na Tabela 7.2 temos o número de ingressantes e a quantidade de evasão até o $10^{\circ}$ semestre por ano, e na Figura 7.1 temos o gráfico da proporção de evasão até o $10^{\circ}$ semestre por ano de ingresso. 
Tabela 7.2: Número e proporção de alunos ingressantes entre 2000 e 2004 que evadiram até o $10^{\circ}$ semestre após o ingresso.

\begin{tabular}{lrrrrr}
\hline Ano de ingresso & 2000 & 2001 & 2002 & 2003 & 2004 \\
\hline Evasão & 1222 & 1022 & 1133 & 1123 & 1094 \\
Ingressantes & 7207 & 7458 & 7993 & 8524 & 8770 \\
\hline Proporção & 0,1696 & 0,1370 & 0,1418 & 0,1318 & 0,1247 \\
\hline
\end{tabular}

\section{Proporção de evasão ao longo dos anos}

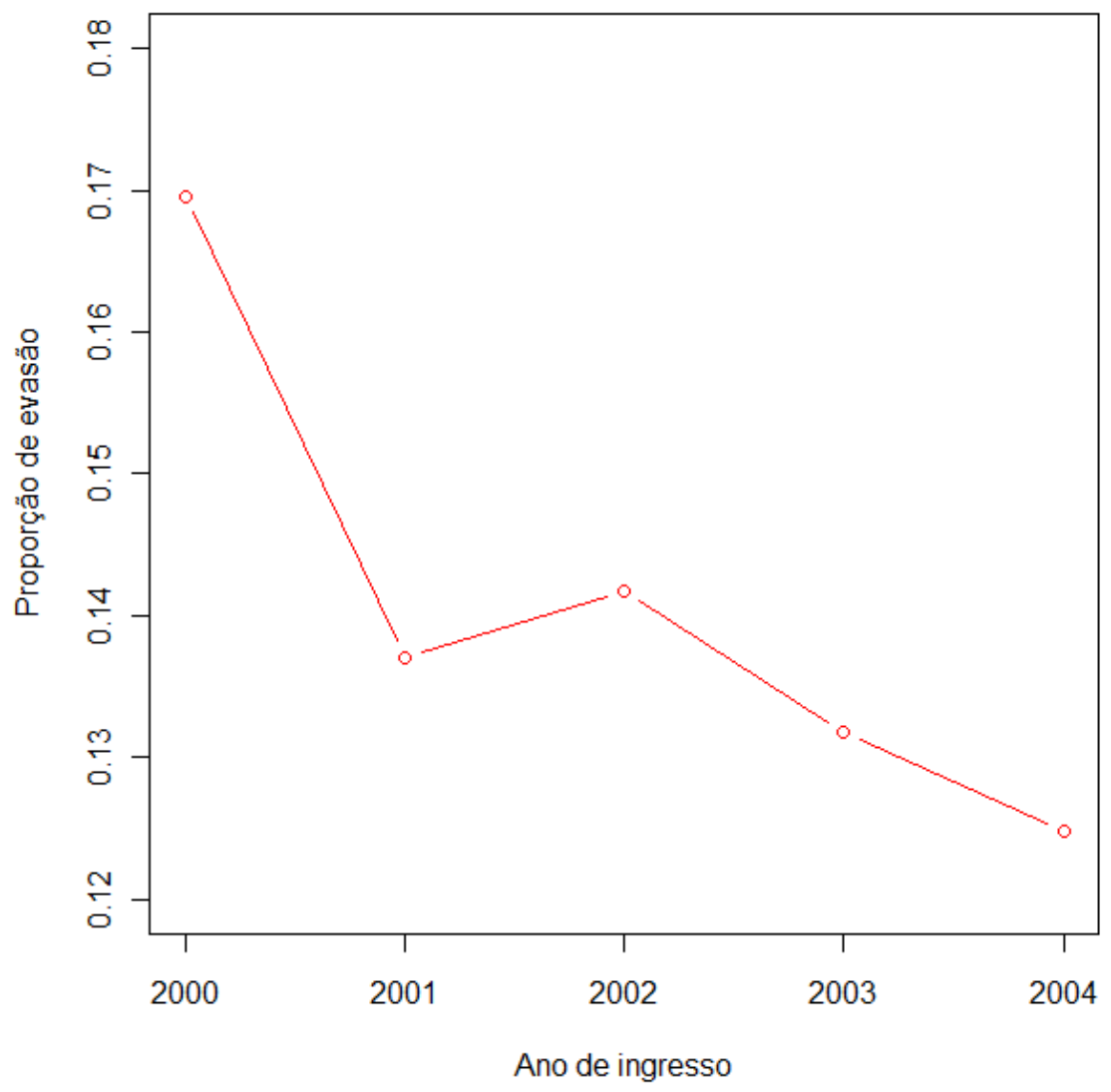

Figura 7.1: Proporção de evasão dos alunos ingressantes em 2000, 2001, 2002, 2003 e 2004 até o $10^{\circ}$ semestre.

Pelo gráfico apresentado na Figura 7.1, verificamos que a evasão vem diminuindo ao longo dos anos. Para verificar se a diferença na evasão ao longo dos anos é significativa, consideremos uma modelo de regressão logística. Os resultados estão na Tabela 7.3. Podemos ver vemos que 
existe diferença significativa entre o ano 2000 e todos os demais, e que a evasão é maior no ano 2000, se comparado com os demais anos.

Tabela 7.3: Resultado do modelo de regressão logística para comparação da evasão ao longo dos anos.

\begin{tabular}{rrrrr}
\hline & Estimador & Desvio Padrão & Estatística do teste & Valor descritivo do teste \\
\hline Intercepto & $-1,58877$ & 0,03139 & $-50,612$ & $2,00 \mathrm{E}-16$ \\
2001 & $-0,25138$ & 0,04604 & $-5,461$ & $4,75 \mathrm{E}-08$ \\
2002 & $-0,21207$ & 0,04488 & $-4,726$ & $2,29 \mathrm{E}-06$ \\
2003 & $-0,29684$ & 0,04484 & $-6,619$ & $3,61 \mathrm{E}-11$ \\
2004 & $-0,35949$ & 0,04505 & $-7,979$ & $1,47 \mathrm{E}-15$ \\
\hline
\end{tabular}

Na Tabela 7.4 temos o número de alunos ingressantes em 2000, 2001, 2002, 2003 ou 2004 que evadiram em cada um dos 10 semestres observados. O semestre com maior número de evasão, dentre os 10 que o aluno tem para evadir, é o $2^{\circ}$ semestre, com 29,7\% da evasão total dos 10 semestres. Aproximadamente $67 \%$ dos alunos evadiram até o $3^{\circ}$ semestre. Na Figura 7.2, um gráfico de pareto apresenta essas informações.

Tabela 7.4: Número de alunos que evadiram por semestre.

\begin{tabular}{cccc}
\hline Semestre & Freqüência & Porcentagem & Porc. Acumalada \\
\hline 2 & 1772 & 29,70 & 29,70 \\
1 & 1616 & 27,08 & 56,78 \\
3 & 560 & 9,38 & 66,16 \\
5 & 416 & 6,97 & 73,14 \\
4 & 398 & 6,67 & 79,81 \\
7 & 312 & 5,23 & 85,03 \\
6 & 307 & 5,14 & 90,18 \\
9 & 220 & 3,69 & 93,87 \\
8 & 213 & 3,57 & 97,44 \\
10 & 153 & 2,56 & 100,00 \\
\hline
\end{tabular}




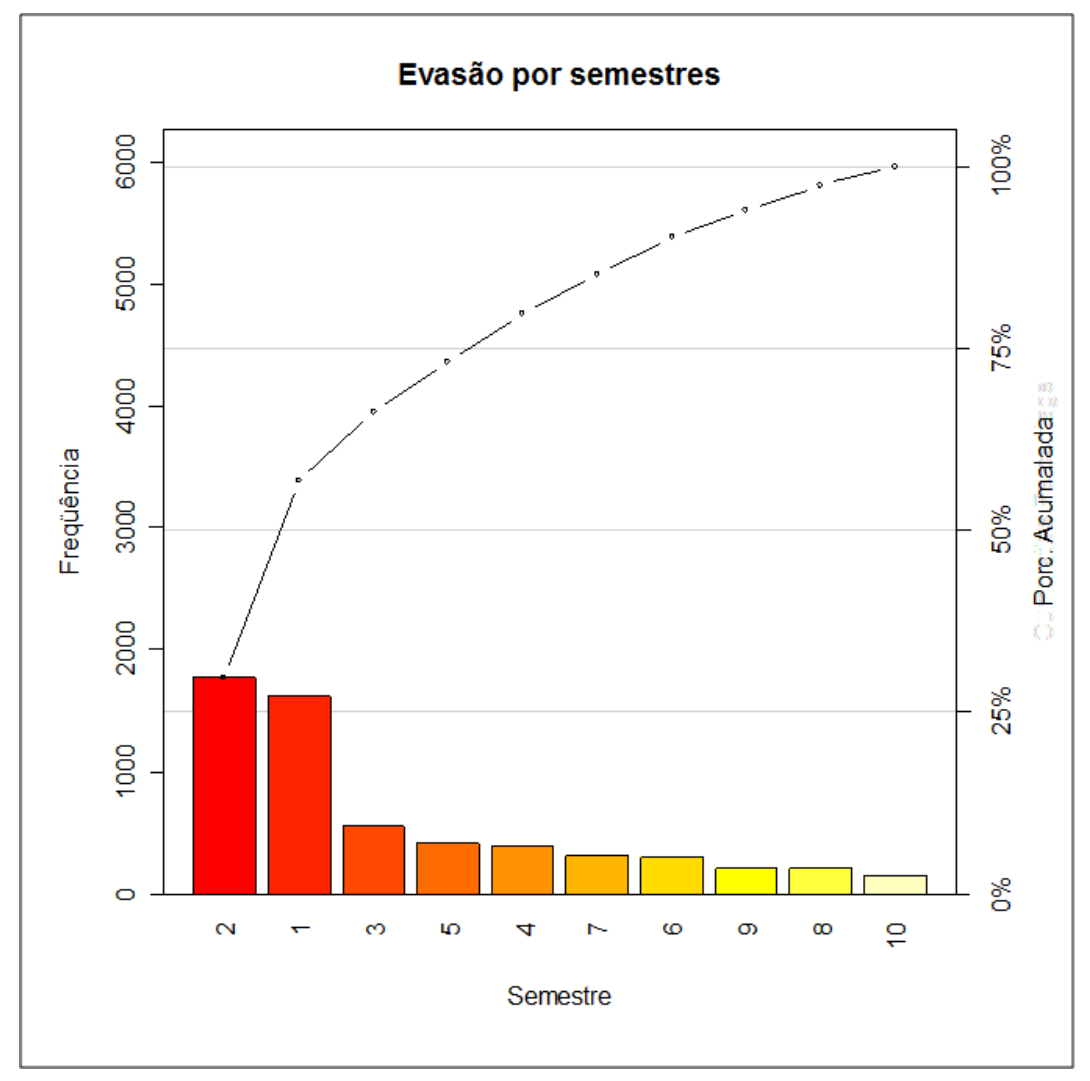

Figura 7.2: Evasão

Há evidência de que variáveis como sexo, período, campus, área estão associadas a ocorrência da evasão e, por isso, serão analisadas a seguir.

Inicialmente analisamos a evasão por sexo. Na Tabela 7.5 temos a proporção de evasão por sexo. A proporção foi calculada em relação ao número de ingressantes daquele sexo no respectivo ano. Na Figura 7.3 vemos que a evasão entre as mulheres é proporcionalmente menor do que entre os homens durante os anos estudados e, em ambos os casos, a evasão diminui ao longo do tempo. 
Tabela 7.5: Proporção de evasão por sexo ao longo dos anos.

\begin{tabular}{crrrrrr}
\hline & \multicolumn{3}{c}{ Feminino } & \multicolumn{3}{c}{ Masculino } \\
Ano de ingresso & Ingressantes & Evasão & Proporção & Ingressantes & Evasão & Proporção \\
\hline 2000 & 3029 & 507 & 0,1674 & 4178 & 974 & 0,2331 \\
2001 & 3262 & 399 & 0,1223 & 4196 & 849 & 0,2023 \\
2002 & 3467 & 420 & 0,1211 & 4562 & 858 & 0,1896 \\
2003 & 3750 & 397 & 0,1059 & 4774 & 782 & 0,1638 \\
2004 & 3684 & 360 & 0,0977 & 5086 & 742 & 0,1459 \\
\hline
\end{tabular}

Proporção de evasão por sexo ao longo dos anos

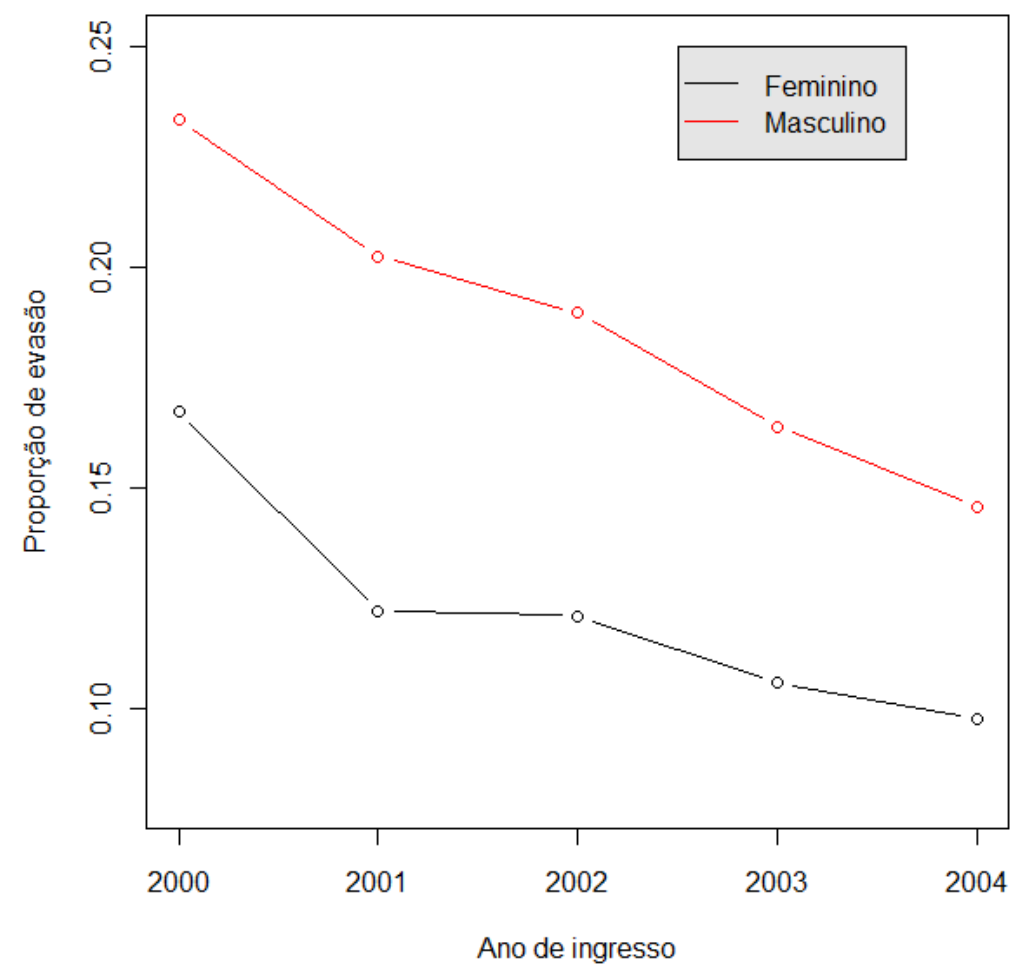

Figura 7.3: Proporção de evasão ao longo dos anos - sexo.

Na Tabela 7.6 apresentamos os valores relativos a evasão por área. Podemos ver que a evasão entre os alunos de biólogicas é proporcionalmente menor do que entre os alunos de humanas ou alunos de exatas.

A proporção de evasão por ano relacionada ao período do curso se encontra na Tabela 7.7. 
Tabela 7.6: Proporção de evasão por área, biológicas, exatas e humanas ao longo dos anos.

\begin{tabular}{crrrrrrrrrr}
\hline & \multicolumn{3}{c}{ Biológicas } & \multicolumn{3}{c}{ Exatas } & \multicolumn{3}{c}{ Humanas } \\
Ano de ingresso & Ingres. & Evasão & Prop. & Ingres. & Evasão & Prop. & Ingres. & Evasão & Prop. \\
\hline 2000 & 1753 & 139 & 0,0793 & 1994 & 419 & 0,2101 & 3460 & 923 & 0,2668 \\
2001 & 1880 & 104 & 0,0553 & 2063 & 476 & 0,1823 & 3515 & 768 & 0,2185 \\
2002 & 2006 & 133 & 0,0663 & 2262 & 382 & 0,1689 & 3725 & 763 & 0,2048 \\
2003 & 2065 & 101 & 0,0489 & 2687 & 385 & 0,1433 & 3772 & 693 & 0,1837 \\
2004 & 2129 & 130 & 0,0611 & 2828 & 371 & 0,1312 & 3813 & 601 & 0,1576 \\
\hline
\end{tabular}

Proporção de evasão por área ao longo dos anos

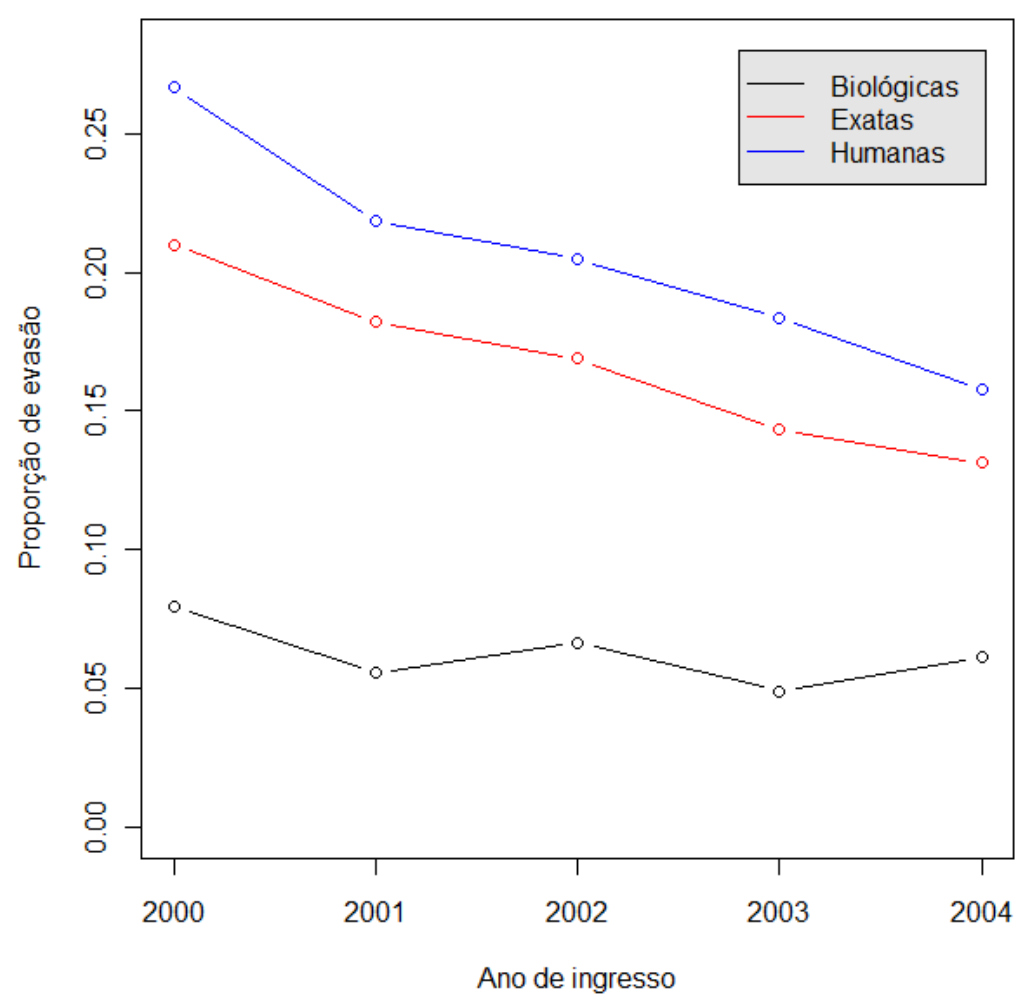

Figura 7.4: Proporção de evasão ao longo dos anos - área.

Da mesma forma, a proporção foi calculada em relação ao número de ingressantes em cada período no respectivo ano. Na Figura 7.5 apresentamos o gráfico da proporção de evasão por período ao longo dos anos. 
Tabela 7.7: Proporção de evasão por período ao longo dos anos.

\begin{tabular}{|c|c|c|c|c|c|c|c|c|c|}
\hline \multirow[b]{2}{*}{ Ano de ingresso } & \multicolumn{3}{|c|}{$\underline{\text { Diurno }}$} & \multicolumn{3}{|c|}{ Integral } & \multicolumn{3}{|c|}{$\underline{\text { Matutino }}$} \\
\hline & Ingres. & Evasão & Prop. & Ingres. & Evasão & Prop. & Ingres. & Evasão & Prop. \\
\hline 2000 & 643 & 159 & 0,2473 & 3032 & 309 & 0,1019 & 763 & 157 & 0,2058 \\
\hline 2001 & 688 & 125 & 0,1817 & 3035 & 259 & 0,0853 & 760 & 155 & 0,2039 \\
\hline 2002 & 740 & 127 & 0,1716 & 3145 & 242 & 0,0769 & 772 & 120 & 0,1554 \\
\hline 2003 & 948 & 128 & 0,1350 & 3390 & 228 & 0,0673 & 767 & 115 & 0,1499 \\
\hline \multirow[t]{2}{*}{2004} & 1111 & 110 & 0,0990 & 3442 & 251 & 0,0729 & 735 & 100 & 0,1361 \\
\hline & \multicolumn{3}{|c|}{ Noturno } & \multicolumn{3}{|c|}{ Vespertino } & & & \\
\hline Ano de ingresso & Ingressantes & Evasão & Proporção & Ingressantes & Evasão & Proporção & & & \\
\hline 2000 & 2432 & 757 & 0,3113 & 337 & 106 & 0,3145 & & & \\
\hline 2001 & 2627 & 612 & 0,2330 & 348 & 97 & 0,2787 & & & \\
\hline 2002 & 2960 & 685 & 0,2314 & 376 & 104 & 0,2766 & & & \\
\hline 2003 & 3067 & 617 & 0,2012 & 352 & 91 & 0,2585 & & & \\
\hline 2004 & 3115 & 546 & 0,1753 & 367 & 95 & 0,2589 & & & \\
\hline
\end{tabular}

Vimos em cada um dos gráficos e tabelas apresentados nesta seção uma aparente diferença na proporção de evasão por sexo, área e período. No entanto, apenas com as informações apresentadas não podemos verificar se essas diferença são significativas. Para isso, na próxima seção, aplicamos o modelo de intensidade multiplicativo ao conjunto de dados de alunos de graduação da USP e realizamos testes de hipóteses para verificar se as diferenças são significativas. 
Proporção de evasão por período ao longo dos anos

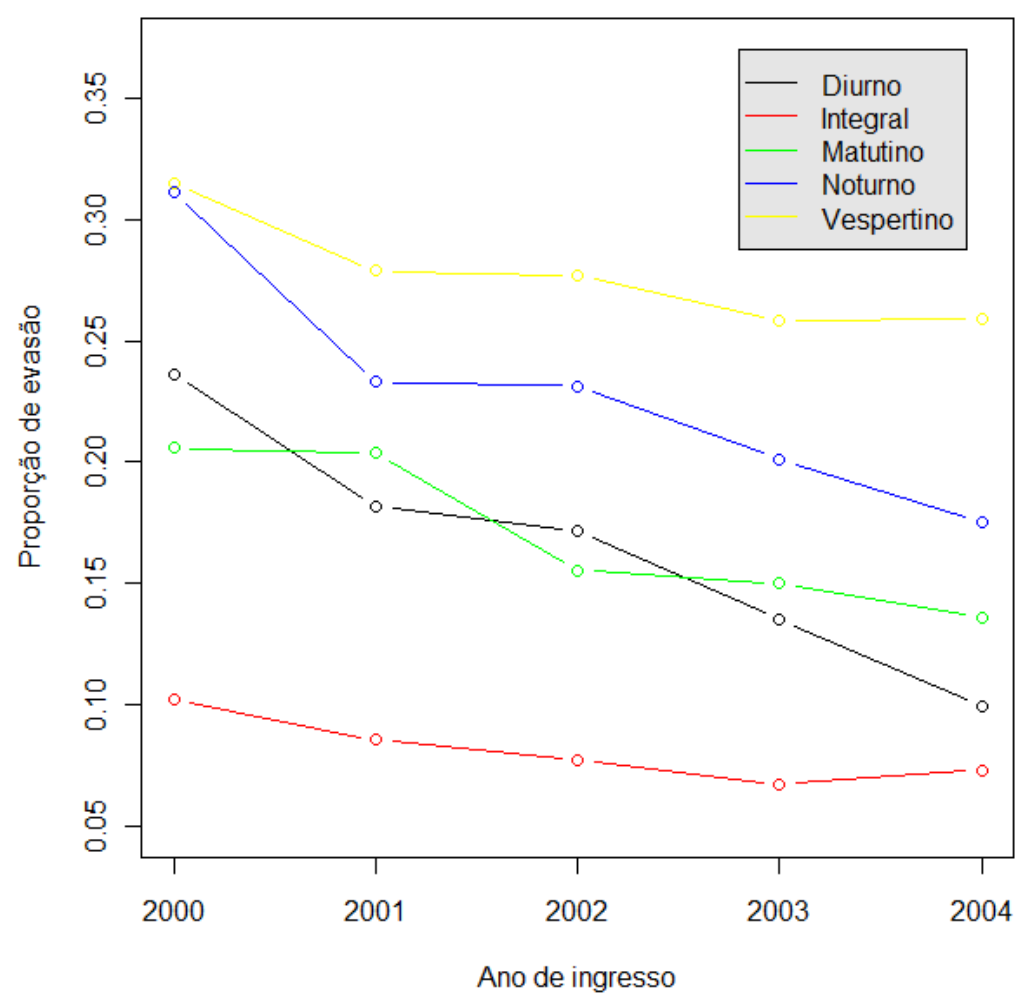

Figura 7.5: Proporção de evasão ao longo dos anos - período.

\subsection{Aplicação do Modelo de Intensidade Multiplicativo}

Nesta seção aplicamos o modelo de intensidade multiplicativo apresentado na Seção 3 aos dados dos alunos de graduação da Universidade de São Paulo. Na seção anterior podemos verificar através do modelo clássido de regressão logística que existe diferença significativa da evasão entre os anos de ingresso e que a evasão vem diminuindo significativamente ao longo dos anos. Utilizando o modelo de intensidade multiplicativo não só conseguimos confirmar esses resultados como também conseguimos identificar se essa diferença entre os anos de ingresso é resultado de uma queda geral da evasão ou se é resultado de uma mudança de comportamento entre semestres. Enquanto o modelo de regressão logística comprara apenas a evasão geral acumulada até o último semestre observado, o modelo de intensidade multiplicativo compara o comportamento da evasão ao longo dos semestres.

Consideremos os alunos ingressantes por vestibular nos anos 2000, 2001, 2003 e 2004. Como 
discutido na Seção 7.2, para fazermos uma análise comparativa da evasão ao longo dos anos consideramos apenas os alunos que evadiram até o $10^{\circ}$ semestre.

A variável aleatória multinomial $X^{(p)}$, assumindo valores $\{1, \ldots, 11\}$ com distribução de probabilidade $\eta^{(p)}$, representa o semestre da última ocorrência de um aluno ingressante no ano $p$, para $p=1, \ldots, 5$ correspondendo aos anos 2000, 2001, 2002, 2003, 2004. Consideremos uma amostra aleatória de tamanho $n_{p}$ desta variável, $X_{1}^{(1)}, \ldots X_{n_{p}}^{(p)}$, em que $n_{p}$ é o número de alunos ingressantes no ano $p$. A variável $X_{m}^{(p)}$, com $m=1, \ldots, n_{p}$, representa o número de semestres até a última ocorrência do aluno $m$ ingressante no ano $p$ e pode ser escrita da seguinte forma

$$
X_{m}^{(p)}=W_{m}^{(p)} \wedge C_{m}^{(p)}
$$

em que $W_{m}^{(p)}$ e $C_{m}^{(p)}$ são variáveis aleatórias multinomiais assumindo valores $\{1, \ldots, 11\}$. Essas variáveis são caracterizadas da seguinte forma,

$$
\begin{gathered}
W_{m}^{(p)}=\left\{\begin{array}{l}
i, \quad \text { se o aluno evadiu no semestre } i, \quad i=1, \ldots, 10 ; \\
11, \quad \text { se o aluno não evadiu até o } 10^{\circ} \text { semestre. }
\end{array}\right. \\
C_{m}^{(p)}=\left\{\begin{array}{l}
i, \quad \text { se o aluno migrou, concluiu ou faleceu no semestre } i, i=1, \ldots, 10 ; \\
11, \quad \text { se não ocorreu nenhum dos eventos de censura até } 10^{\circ} \text { semestre. }
\end{array}\right.
\end{gathered}
$$

Para esta aplicação, o processo $N^{n_{p}}(i,$.$) definido na Seção 3.3$ pela seguinte equação

$$
N^{n_{p}}(i, .)=\sum_{m=1}^{n_{p}} N_{m}^{(p)}(i, .)
$$

indica quantos alunos ingressantes no ano $p$ evadiram até o semestre $i, i=1, \ldots, 10$. Portanto, $\Delta N^{n_{p}}(i,)=.N^{n_{p}}(i,)-.N^{n_{p}}(i-1,$.$) indica o número de alunos que evadiram no semestre i$. O processo $V^{n_{p}}(i,$.$) representa o número de alunos ingressantes no ano p$ sob risco no $i$-ésimo semestre.

A seguir, utilizando o modelo de intensidade multiplicativo, comparamos a evasão ao longo dos anos e sua relação com as variáveis sexo, período e área.

\subsubsection{Comparação entre anos}

Nas Tabelas 7.8 e 7.9 as colunas intituladas "Sob risco" e "Evasão" correspondem, respectivamente, ao número de alunos sob risco e que evadiram em cada semestre. A coluna "Outros" 
corresponde a quantidade de vezes por semestre em que ocorreu os eventos de censura migração, falecimento e transferência. Utilizando esse valores, obtemos os estimadores dos parâmetros do modelo de intensidade multiplicativo.

Para exclarecer como são efetuados os cálculos para se obter os estimadores dos parâmetros do modelo de intensidade multiplicativo, consideremos como exemplo o ano de $2003(p=4)$. Temos que $\Delta N^{(4)}(1,)=$.212 é o número de alunos que evadiram no $1^{o}$ semestre e $\Delta N^{(4)}(2,)=$. 228 é o número de alunos que evadiram no $2^{o}$ semestre. $V^{(4)}(1,)=$.8522 é o número de alunos sob risco no semestre 1 . No primeiro semestre um evento de censura ocorreu apenas uma única vez. Portanto, o número de alunos sob risco no segundo semestre é $V^{(4)}(2,)=.8522-(212+1)=$ 8309.

Através destes valores e da equação 3.13 obtemos o valor estimado da função intensidade $\hat{h}^{n_{4}}$ referente ao ano de 2003 e ao semestre 1,

$$
\hat{h}^{n_{4}}(1)=\frac{\Delta N^{n_{4}}(1, .)}{V^{n_{4}}(1, .)}=\frac{212}{8522}=0,02487
$$

Para os demais anos e semestres os cálculos são efetuados de forma análoga.

Depois de calculados os valores de $\hat{h}^{n_{p}}$, obtemos as estimativas da função intensidade acumulada e da distribuição de probabilidade, utilizando as equações

$$
\hat{H}^{n_{p}}(i)=\sum_{l=1}^{i} \hat{h}^{n_{p}}(i)
$$

e

$$
\hat{\zeta}^{n_{p}}(i)=\hat{h}^{n_{p}}(i) \prod_{l=1}^{i-1} \hat{h}^{n_{p}}(l)
$$

Nas Tabelas 7.10, 7.11 e 7.12 temos as estimativas da função intensidade, da função intensidade acumulada, da probabilidade de evasão e da probabilidade acumulada por ano para cada um dos 10 semestres analisados. 
Tabela 7.8: Número de alunos que estavam sob risco, que evadiram, ou que tiveram outro tipo de última ocorrência em cada um dos 10 semestres analisados, por ano de ingresso.

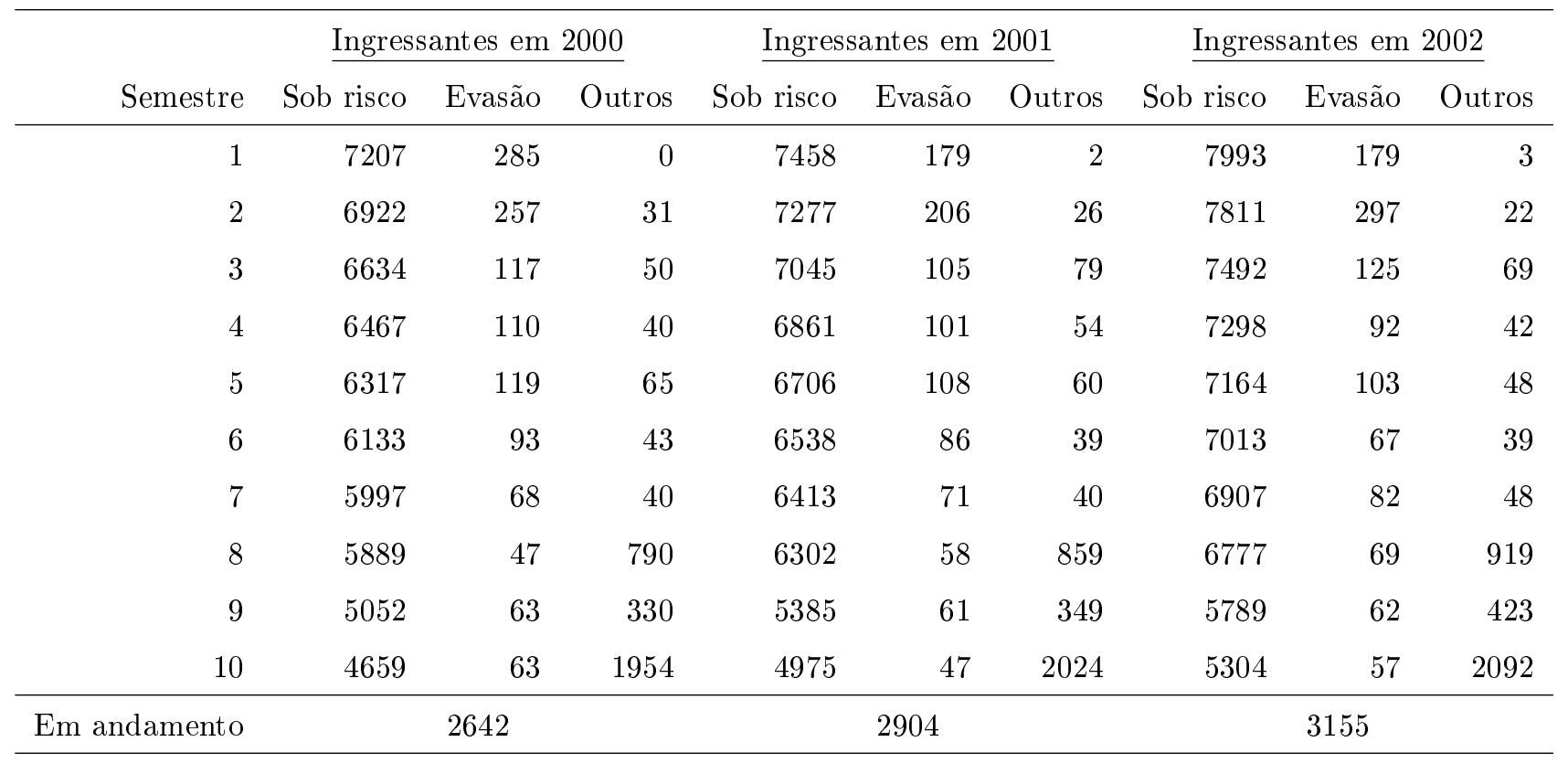

Tabela 7.9: Número de alunos que estavam sob risco, que evadiram, ou que tiveram outro tipo de última ocorrência em cada um dos 10 semestres analisados, por ano de ingresso.

\begin{tabular}{rrrrrrrr}
\hline & \multicolumn{3}{c}{ Ingressantes em 2003 } & & \multicolumn{3}{c}{ Ingressantes em 2004 } \\
\cline { 3 - 7 } Semestre & Sob risco & Evasão & Outros & Sob risco & Evasão & Outros \\
\hline 1 & 8524 & 212 & 1 & 8770 & 194 & 0 \\
2 & 8311 & 228 & 33 & 8576 & 279 & 13 \\
3 & 8050 & 117 & 90 & 8284 & 103 & 82 \\
4 & 7843 & 104 & 60 & 8099 & 98 & 44 \\
5 & 7679 & 100 & 52 & 7957 & 130 & 65 \\
6 & 7527 & 60 & 45 & 7762 & 92 & 45 \\
7 & 7422 & 105 & 48 & 7625 & 90 & 67 \\
8 & 7269 & 79 & 1021 & 7468 & 54 & 1009 \\
9 & 6169 & 79 & 405 & 6405 & 47 & 433 \\
10 & 5685 & 39 & 2141 & 5925 & 7 & 1956 \\
\hline Em andamento & 3505 & & & 3964 & \\
\hline
\end{tabular}


Tabela 7.10: Estimativas da função intensidade, da função intensidade acumulada, da probabilidade de evasão e da probabilidade acumulada em cada um dos 10 semestres analisados, por ano de ingresso, 2000 e 2001.

\begin{tabular}{rrrrrrrrrr}
\hline & \multicolumn{4}{c}{ Ingressantes em 2000 } & \multicolumn{3}{c}{ Ingressantes em 2001 } \\
\cline { 8 - 11 } Semestre $(l)$ & $h(l)$ & $H(l)$ & $\zeta(l)$ & Acum. & $h(l)$ & $H(l)$ & $\zeta(l)$ & Acum. \\
\hline 1 & 0,0395 & 0,0395 & 0,0395 & 0,0395 & 0,0240 & 0,0240 & 0,0240 & 0,0240 \\
2 & 0,0371 & 0,0767 & 0,0357 & 0,0752 & 0,0283 & 0,0523 & 0,0276 & 0,0516 \\
3 & 0,0169 & 0,0936 & 0,0156 & 0,0908 & 0,0145 & 0,0668 & 0,0138 & 0,0654 \\
4 & 0,0156 & 0,1092 & 0,0142 & 0,1050 & 0,0139 & 0,0807 & 0,0130 & 0,0784 \\
5 & 0,0169 & 0,1261 & 0,0151 & 0,1201 & 0,0148 & 0,0955 & 0,0136 & 0,0920 \\
6 & 0,0132 & 0,1393 & 0,0117 & 0,1318 & 0,0118 & 0,1073 & 0,0107 & 0,1027 \\
7 & 0,0096 & 0,1489 & 0,0083 & 0,1401 & 0,0097 & 0,1170 & 0,0087 & 0,1114 \\
8 & 0,0066 & 0,1556 & 0,0057 & 0,1458 & 0,0079 & 0,1249 & 0,0070 & 0,1184 \\
9 & 0,0099 & 0,1654 & 0,0084 & 0,1543 & 0,0093 & 0,1342 & 0,0082 & 0,1267 \\
10 & 0,0092 & 0,1747 & 0,0078 & 0,1621 & 0,0067 & 0,1409 & 0,0058 & 0,1325 \\
\hline
\end{tabular}

Tabela 7.11: Estimativas da função intensidade, da função intensidade acumulada, da probabilidade e da probabilidade acumulada em cada um dos 10 semestres analisados, por ano de ingresso, 2002 e 2003.

\begin{tabular}{rrrrrrrrr}
\hline \multicolumn{4}{c}{ Ingressantes em 2002 } & \multicolumn{3}{c}{ Ingressantes em 2003 } \\
\hline Semestre $(l)$ & $h(l)$ & $H(l)$ & $\zeta(l)$ & Acum. & $h(l)$ & $H(l)$ & $\zeta(l)$ & Acum. \\
\hline 1 & 0,0224 & 0,0224 & 0,0224 & 0,0224 & 0,0249 & 0,0249 & 0,0249 & 0,0249 \\
2 & 0,0380 & 0,0604 & 0,0372 & 0,0596 & 0,0274 & 0,0523 & 0,0268 & 0,0516 \\
3 & 0,0163 & 0,0767 & 0,0153 & 0,0749 & 0,0142 & 0,0665 & 0,0134 & 0,0651 \\
4 & 0,0118 & 0,0885 & 0,0109 & 0,0858 & 0,0125 & 0,0790 & 0,0117 & 0,0767 \\
5 & 0,0131 & 0,1016 & 0,0120 & 0,0978 & 0,0120 & 0,0909 & 0,0110 & 0,0878 \\
6 & 0,0085 & 0,1102 & 0,0077 & 0,1055 & 0,0072 & 0,0981 & 0,0065 & 0,0943 \\
7 & 0,0104 & 0,1205 & 0,0093 & 0,1148 & 0,0125 & 0,1106 & 0,0113 & 0,1056 \\
8 & 0,0088 & 0,1293 & 0,0078 & 0,1226 & 0,0094 & 0,1200 & 0,0084 & 0,1141 \\
9 & 0,0089 & 0,1382 & 0,0078 & 0,1303 & 0,0106 & 0,1306 & 0,0094 & 0,1235 \\
10 & 0,0076 & 0,1458 & 0,0066 & 0,1369 & 0,0049 & 0,1355 & 0,0043 & 0,1277 \\
\hline
\end{tabular}


Tabela 7.12: Estimativas da função intensidade, da função intensidade acumulada, da probabilidade e da probabilidade acumulada em cada um dos 10 semestres analisados, por ano de ingresso, 2004.

\begin{tabular}{rrrrr}
\hline \multicolumn{5}{c}{ Ingressantes em 2004 } \\
\cline { 3 - 6 } Semestre $(l)$ & $h(l)$ & $H(l)$ & $\zeta(l)$ & Acum. \\
\hline 1 & 0,0221 & 0,0221 & 0,0221 & 0,0221 \\
2 & 0,0325 & 0,0547 & 0,0318 & 0,0539 \\
3 & 0,0121 & 0,0668 & 0,0115 & 0,0654 \\
4 & 0,0114 & 0,0782 & 0,0107 & 0,0761 \\
5 & 0,0151 & 0,0933 & 0,0139 & 0,0900 \\
6 & 0,0107 & 0,1040 & 0,0098 & 0,0998 \\
7 & 0,0104 & 0,1144 & 0,0094 & 0,1092 \\
8 & 0,0063 & 0,1207 & 0,0056 & 0,1147 \\
9 & 0,0061 & 0,1268 & 0,0054 & 0,1201 \\
10 & 0,0008 & 0,1277 & 0,0007 & 0,1209 \\
\hline
\end{tabular}

Estimador da função intensidade $\mathrm{h}$

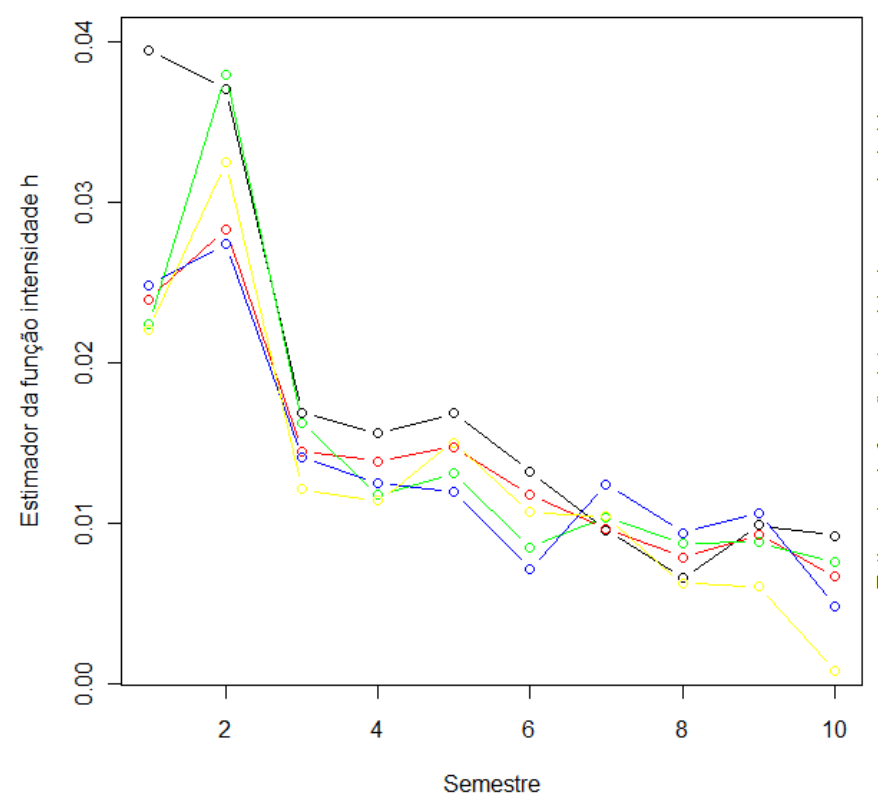

Estimador da função intensidade acumulada $\mathrm{H}$

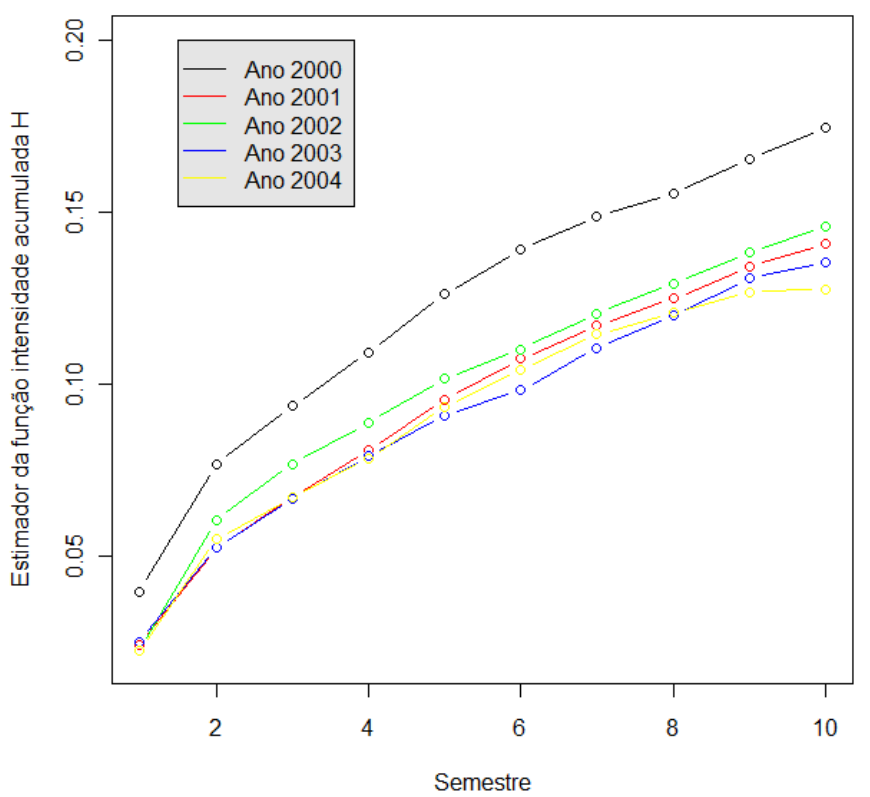

Figura 7.6: Estimadores da função intensidade e da função intensidade acumualada por semestres ao longo dos anos. 


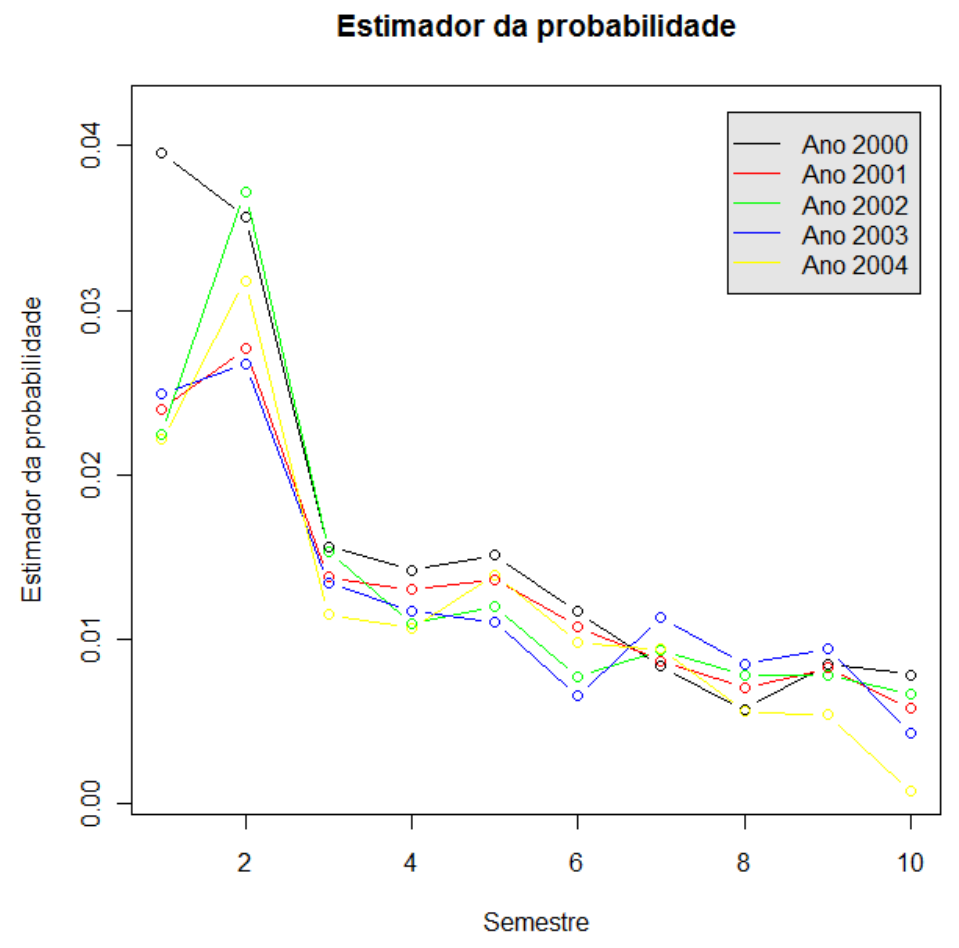

Figura 7.7: Probabilidade de evasão por semestre de alunos ingressantes em 2000, 2001, 2002 e 2003. 


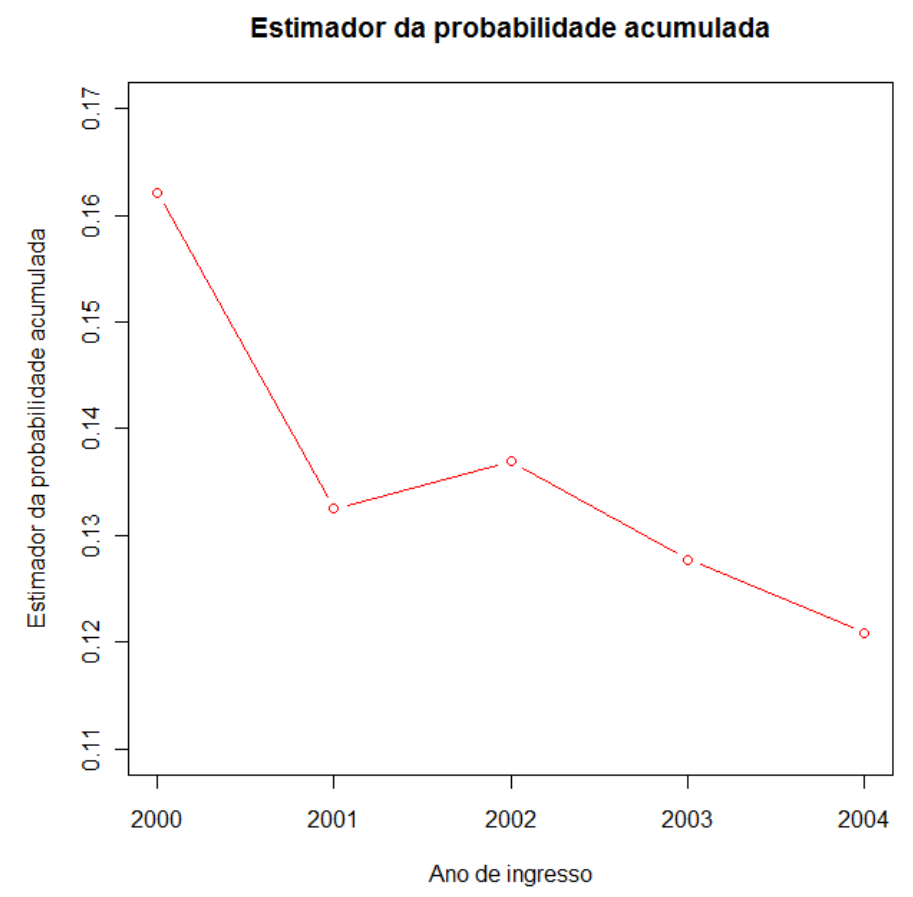

Figura 7.8: Probabilidade de evasão por semestre de alunos ingressantes em 2000, 2001, 2002 e 2003.

Para compararmos a evasão entre os anos de ingresso, utilizamos a estatística de logrank apresentada na Capítulo 5 para comparação de duas populações. Nosso interesse é comparar o comportamento da evasão entre alunos ingressantes em dois anos diferentes. Assim, nosso interesse é testar as seguintes hipóteses

$$
\left\{\begin{array}{l}
H_{0}: H^{(t)}=H^{(p)} \\
H_{1}: H^{(t)} \neq H^{(p)}
\end{array}\right.
$$

A estatística de teste utilizada é dada por

$$
W=\sum_{l=1}^{i} U^{n_{t}, n_{p}}(l, .)\left[\hat{h}^{n_{t}}(l)-\hat{h}^{n_{p}}(l)\right]
$$

em que

$$
U^{n_{t}, n_{p}}(l, .)=\sqrt{\left(\frac{n_{t}+n_{p}}{n_{t} n_{p}}\right)} \frac{V^{n_{t}}(l, .) V^{n_{p}}(l, .)}{V(l, .)}
$$

A estatística $W$ tem distribuição normal com média 0 e variância

$$
\hat{\sigma}^{2}(i)=\sum_{l=1}^{i} \frac{U^{n_{t}, n_{p} 2}(l, .)}{V^{n_{t}}(l, .)}\left[1-\hat{h}^{n_{t}}(l)\right] \hat{h}^{n_{t}}(l)+\sum_{l=1}^{i} \frac{U^{n_{t}, n_{p} 2}(l, .)}{V^{n_{p}}(l, .)}\left[1-\hat{h}^{n_{p}}(l)\right] \hat{h}^{n_{p}}(l) .
$$


Com isso, obtemos as estatísticas de testes resultantes da comparação entres os anos e os respectivos valores descritivos do teste. Estes valores são apresentados na Tabela 7.13.

Tabela 7.13: Testes de hipóteses de comparação dos anos.

\begin{tabular}{rrr}
\hline & Estatística & Valor descritivo do teste \\
\hline $2000-2001$ & 5,17 & $2,31 \mathrm{E}-07$ \\
$2000-2002$ & 4,49 & $7,29 \mathrm{E}-06$ \\
$2000-2003$ & 6,21 & $5,09 \mathrm{E}-10$ \\
$2000-2004$ & 7,51 & $5,98 \mathrm{E}-14$ \\
$2001-2002$ & $-0,79$ & 0,427 \\
$2001-2003$ & 0,92 & 0,358 \\
$2001-2004$ & 2,20 & 0,028 \\
$2002-2003$ & 1,76 & 0,079 \\
$2002-2004$ & 3,06 & 0,0022 \\
$2003-2004$ & 1,32 & 0,187 \\
\hline
\end{tabular}

Como realizamos 10 testes de comparação dois a dois, utilizaremos o critério de Bonferroni para definirmos o nível de significância das comparações. Com isso, admitimos um nível de significância de 0,005 .

Com este nível de significância, concluímos que existe diferença siginificativa na evasão entre o ano 2000 e todos os demais anos, pois os valores descritivos dos testes são menores que 0,005. Além disso, as estatísticas de teste são positivas, o que implica que a evasão é sempre maior para alunos ingressantes em 2000 quando comparada a alunos ingressantes nos demais anos. Observando a Figura 7.6, podemos verificar que a diferença da evasão entre 2000 e os demais anos não é só resultante de uma queda na evasão ao longo dos anos, mas também de uma mudança de comportamento da evasão durante os 10 semestres analisados. Observando a Figura 7.9, podemos ver que para o ano 2000 temos o mais alto índice de evasão no primeiro semestre, decaindo no segundo e mais ainda no terceiro. No entanto, para os anos 2001 e 2004, a evasão no segundo semestre é maior do que no primeiro.

Há diferença siginificativa também na evasão entre os anos 2002 e 2004 pois o valor descritivo do teste é menor do que 0,005. Além disso, como a estatística de teste é positiva, concluímos 

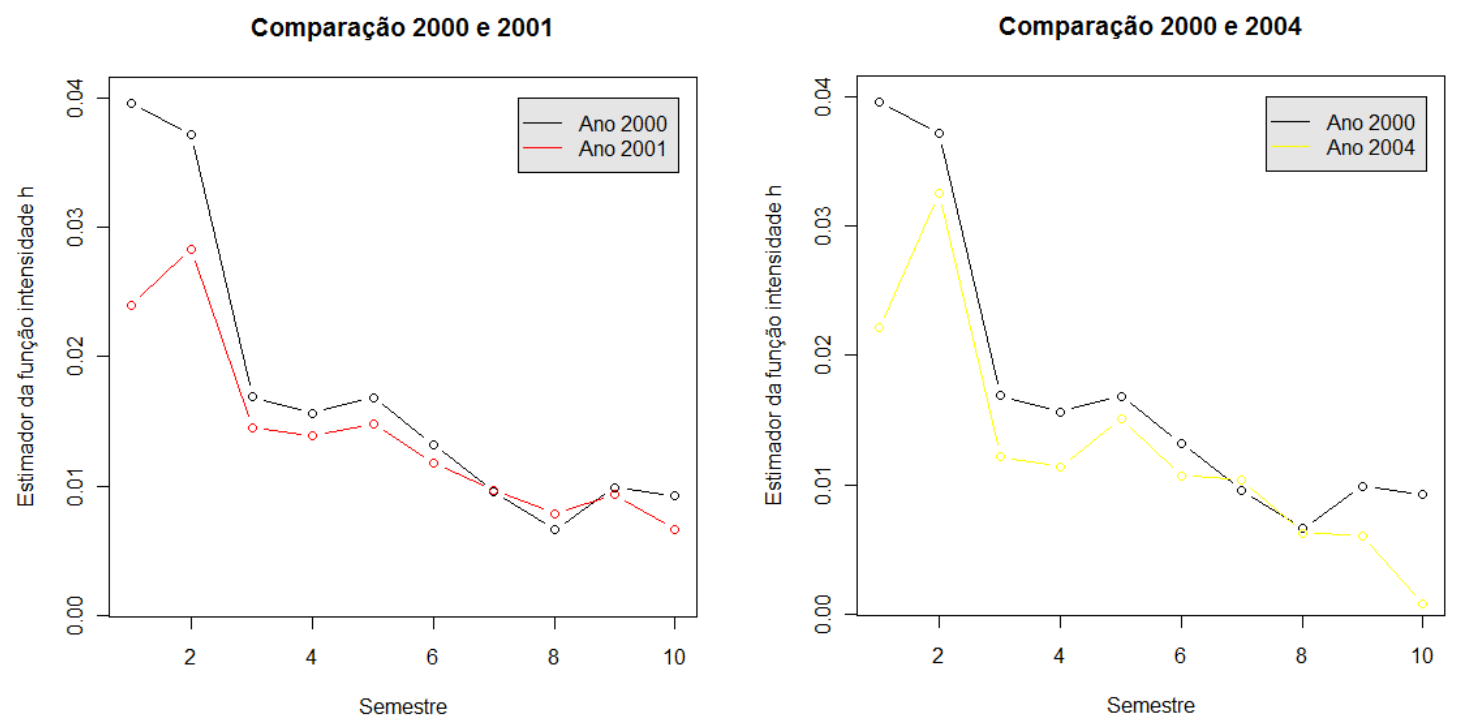

Figura 7.9: Probabilidade de evasão por semestre de alunos ingressantes em 2000, 2001, 2002 ou 2003.

que a evasão decaiu de 2002 para 2004 e, observando a Figura 7.6, confirmamos que a diferença é resultante apenas dessa queda da evasão pois não há mudança de comportamento ao longo dos 10 semestres para os ingressos em 2002 e 2004. Entre os demais anos, não existe diferença significativa.

\subsubsection{Comparação entre sexos}

Realizamos agora uma análise comparativa da evasão entre sexo feminino e masculino. Na Tabela 7.14 temos as estimativas da função intesnsidade, da função intensidade acumulada e da distribuição de probabilidade para sexo masculino e feminino em cada uma das 10 categorias. Na Figura 7.10, temos os gráficos da função intensidade e da função intensidade acumulada para os sexos.

Nosso interesse é verificar se existe diferença significativa da evasão entre homens e mulheres. Para isso, vamos testar as seguintes hipóteses

$$
\left\{\begin{array}{l}
H_{0}: H^{(1)}=H^{(2)} \\
H_{1}: H^{(1)} \neq H^{(2)}
\end{array}\right.
$$

em que $H^{(1)}$ é a função intensidade referente à população de mulheres e $H^{(2)}$ é a função inten- 
Tabela 7.14: Estimadores da função intensidade, da função intensidade acumulada, da probabilidade e da probabilidade acumulada em cada um dos 10 semestres analisados por sexo.

\begin{tabular}{rrrrrrrrr}
\hline \multicolumn{1}{c}{ Feminino } \\
1 & $h(l)$ & $H(l)$ & $\zeta(l)$ & Acumulada & $h(l)$ & $H(l)$ & $\zeta(l)$ & Acumulada \\
\hline 1 & 0,01849 & 0,01849 & 0,01849 & 0,01849 & 0,02949 & 0,02949 & 0,02949 & 0,02949 \\
2 & 0,02270 & 0,04119 & 0,02228 & 0,04077 & 0,03962 & 0,06911 & 0,03845 & 0,06794 \\
3 & 0,01239 & 0,05358 & 0,01189 & 0,05265 & 0,01664 & 0,08575 & 0,01551 & 0,08345 \\
4 & 0,00760 & 0,06118 & 0,00720 & 0,05986 & 0,01283 & 0,09858 & 0,01176 & 0,09521 \\
5 & 0,00942 & 0,07061 & 0,00886 & 0,06872 & 0,01430 & 0,11287 & 0,01294 & 0,10814 \\
6 & 0,00565 & 0,07626 & 0,00526 & 0,07398 & 0,00850 & 0,12138 & 0,00759 & 0,11573 \\
7 & 0,00564 & 0,08190 & 0,00522 & 0,07920 & 0,00912 & 0,13050 & 0,00807 & 0,12379 \\
8 & 0,00461 & 0,08651 & 0,00425 & 0,08345 & 0,00603 & 0,13653 & 0,00529 & 0,12908 \\
9 & 0,00462 & 0,09113 & 0,00423 & 0,08768 & 0,00633 & 0,14286 & 0,00552 & 0,13459 \\
10 & 0,00321 & 0,09434 & 0,00293 & 0,09061 & 0,00437 & 0,14723 & 0,00378 & 0,13837 \\
\hline
\end{tabular}

Função intensidade - sexo

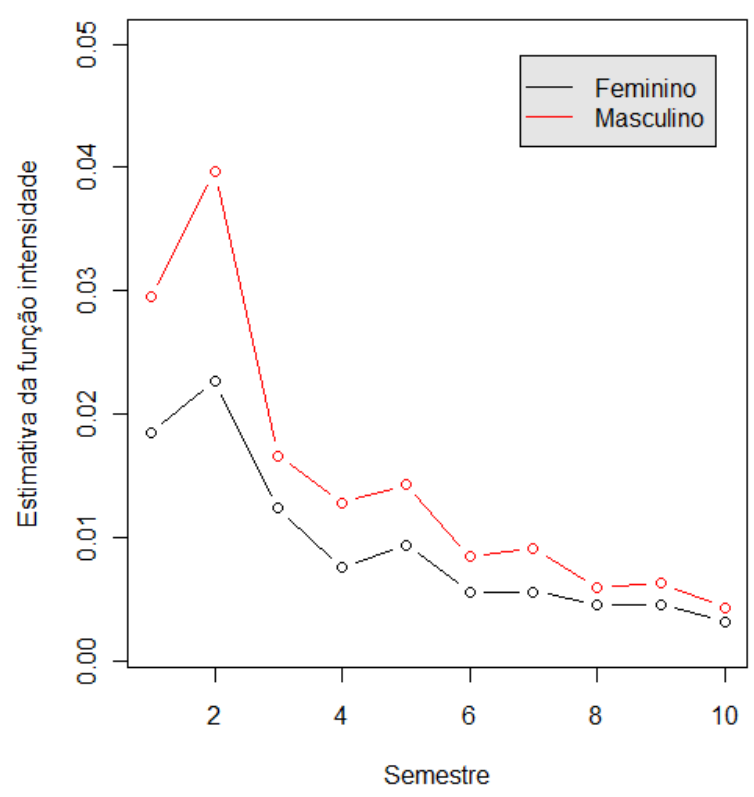

Função intensidade acumulada - sexo

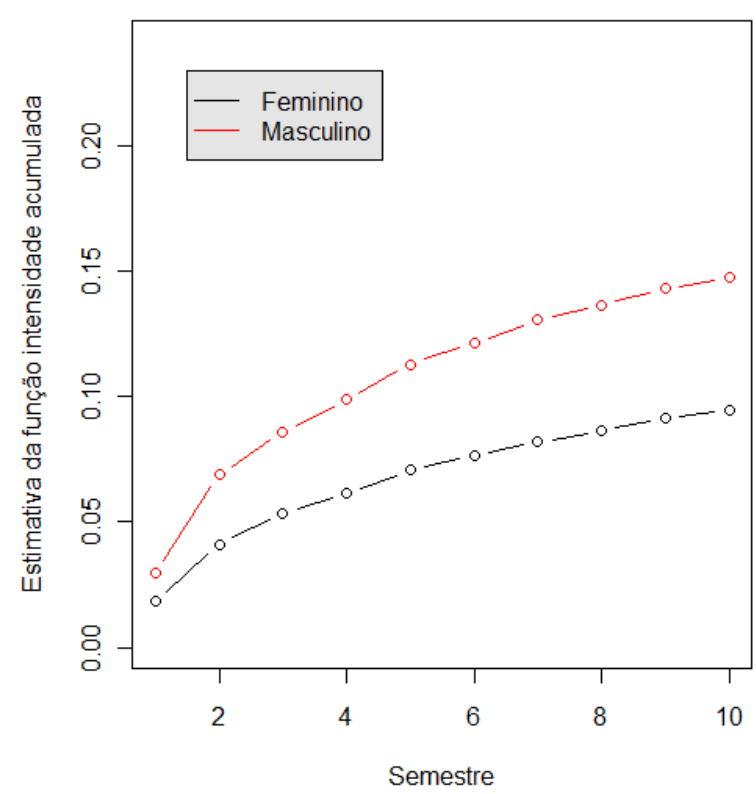

Figura 7.10: Estimadores da função intensidade e da função intensidade acumualada por semestres - sexo. 
sidade acumulada referente à população de homens. Para testar estas hipóteses utilizamos a estatística de teste

$$
W=\sum_{l=1}^{i} U^{n_{1}, n_{2}}(l, .)\left[\hat{h}^{n_{1}}(l)-\hat{h}^{n_{2}}(l)\right]
$$

em que

$$
U^{n_{1}, n_{2}}(l, .)=\sqrt{\left(\frac{n_{1}+n_{2}}{n_{1} n_{2}}\right)} \frac{V^{n_{1}}(l, .) V^{n_{2}}(l, .)}{V(l, .)}
$$

A estatística $W$ que tem distribuição normal com média 0 e variância

$$
\hat{\sigma}^{2}(i)=\sum_{l=1}^{i} \frac{U^{n_{1}, n_{2} 2}(l, .)}{V^{n_{1}}(l, .)}\left[1-\hat{h}^{n_{1}}(l)\right] \hat{h}^{n_{1}}(l)+\sum_{l=1}^{i} \frac{U^{n_{1}, n_{2} 2}(l, .)}{V^{n_{2}}(l, .)}\left[1-\hat{h}^{n_{2}}(l)\right] \hat{h}^{n_{2}}(l) .
$$

Obtemos o resultado apresentado na Tabela 7.15. Concluímos, portanto, que existe diferença significativa da evasão entre homens e mulheres, e que a evasão entre mulheres é menor do que entre os homens. Além disso, observando o grafico 7.10, vemos que não há mudança de comportamento ao longo dos 10 semestres entre homens e mulheres. Portanto, a diferença está apenas no fato da evasão ser menor entre mulheres.

Tabela 7.15: Teste de comparação entre sexos.

\begin{tabular}{lrr}
\hline Sexo & Estatística & Valor descritivo do teste \\
\hline Feminino/Maculino & $-22,24$ & $1,29 \mathrm{E}-109$ \\
\hline
\end{tabular}

\subsubsection{Comparação entre períodos}

Agora, realizamos teste de comparação entre os períodos de cursos. Os períodos existentes são:

- Diurno: curso pode ser ministrado de manhã e de tarde.

- Integral: curso pode ser ministrado durante todo o dia: manhã, tarde e noite.

- Matutino: curso ministrado apenas de manhã.

- Noturno: curso ministrado apenas a noite.

- Vespertino: curso ministrado apenas a tarde. 
Nas Tabelas 7.16, 7.17 e 7.18 temos as estimativas da função intensidade, da função intensidade acumulada e da probabilidade para cada um dos períodos.

Tabela 7.16: Estimativas da função intensidade, da função intensidade acumulada, da probabilidade e da probabilidade acumulada em cada um dos 10 semestres analisados por periodo Diurno, integral.

\begin{tabular}{rrrrrrrrr}
\hline \multicolumn{4}{c}{ Diurno } & \multicolumn{5}{c}{ Integral } \\
$l$ & $h(l)$ & $H(l)$ & $\zeta(l)$ & Acumulada & $h(l)$ & $H(l)$ & $\zeta(l)$ & Acumulada \\
\hline 1 & 0,0211 & 0,0211 & 0,0211 & 0,0211 & 0,0086 & 0,0086 & 0,0086 & 0,0086 \\
2 & 0,0311 & 0,0522 & 0,0305 & 0,0516 & 0,0145 & 0,0230 & 0,0143 & 0,0229 \\
3 & 0,0132 & 0,0655 & 0,0126 & 0,0641 & 0,0090 & 0,0320 & 0,0088 & 0,0317 \\
4 & 0,0081 & 0,0736 & 0,0076 & 0,0717 & 0,0051 & 0,0371 & 0,0049 & 0,0366 \\
5 & 0,0126 & 0,0862 & 0,0117 & 0,0834 & 0,0075 & 0,0446 & 0,0072 & 0,0438 \\
6 & 0,0064 & 0,0926 & 0,0059 & 0,0893 & 0,0034 & 0,0480 & 0,0033 & 0,0471 \\
7 & 0,0070 & 0,0996 & 0,0064 & 0,0957 & 0,0046 & 0,0526 & 0,0043 & 0,0514 \\
8 & 0,0051 & 0,1046 & 0,0046 & 0,1002 & 0,0027 & 0,0553 & 0,0026 & 0,0540 \\
9 & 0,0059 & 0,1105 & 0,0053 & 0,1055 & 0,0035 & 0,0588 & 0,0033 & 0,0573 \\
10 & 0,0048 & 0,1154 & 0,0043 & 0,1099 & 0,0023 & 0,0611 & 0,0022 & 0,0595 \\
\hline
\end{tabular}

O interesse aqui é verificar se existe diferença significativa entre os períodos e identificar em quais períodos a evasão é maior. Para isso, testamos as hipóteses

$$
\left\{\begin{array}{l}
H_{0}: H^{(t)}=H^{(p)} \\
H_{1}: H^{(t)} \neq H^{(p)}
\end{array}\right.
$$

em que $t$ e $p$ correspondem a quaisquer diferentes períodos. Utilizando a estatística já apresentada nesta seção para comparação dos anos, obtemos os resultados apresentados na Tabela 7.19 .

Utilizando o critério de Bonferroni, adotamos um nível de significância de 0,005. Com isso, concluímos que apenas entre os período noturno e vespertino não existe diferença significativa. Entre todos os outros há diferença significativa. Observando a Figura 7.11 concluímos que as diferenças existentes são resultantes apenas da queda de evasão entre os período e não de uma variação no comportamento ao longo dos semestres. Concluímos também que a evasão é maior nos períodos noturno e vespertino, seguido dos períodos matutino, diurno e integral. 
Tabela 7.17: Estimadores da função intensidade, da função intensidade acumulada, da probabilidade e da probabilidade acumulada em cada um dos 10 semestres analisados por período Matutino, vespertino.

\begin{tabular}{rrrrrrrrr}
\hline & \multicolumn{9}{c}{ Matutino } & & \multicolumn{3}{c}{ Vespertino } \\
\cline { 6 - 9 } \\
\hline 1 & 0,0302 & 0,0302 & 0,0302 & 0,0302 & 0,0446 & 0,0446 & 0,0446 & 0,0446 \\
2 & 0,0381 & 0,0683 & 0,0370 & 0,0672 & 0,0458 & 0,0905 & 0,0438 & 0,0884 \\
3 & 0,0149 & 0,0832 & 0,0139 & 0,0811 & 0,0297 & 0,1202 & 0,0271 & 0,1155 \\
4 & 0,0117 & 0,0949 & 0,0107 & 0,0918 & 0,0173 & 0,1375 & 0,0153 & 0,1308 \\
5 & 0,0133 & 0,1082 & 0,0121 & 0,1039 & 0,0159 & 0,1534 & 0,0139 & 0,1447 \\
6 & 0,0080 & 0,1162 & 0,0071 & 0,1110 & 0,0096 & 0,1630 & 0,0082 & 0,1529 \\
7 & 0,0053 & 0,1215 & 0,0047 & 0,1157 & 0,0115 & 0,1746 & 0,0098 & 0,1627 \\
8 & 0,0048 & 0,1262 & 0,0042 & 0,1200 & 0,0084 & 0,1829 & 0,0070 & 0,1697 \\
9 & 0,0051 & 0,1313 & 0,0045 & 0,1244 & 0,0081 & 0,1910 & 0,0067 & 0,1764 \\
10 & 0,0028 & 0,1341 & 0,0024 & 0,1268 & 0,0064 & 0,1974 & 0,0052 & 0,1816 \\
\hline
\end{tabular}

Tabela 7.18: Estimativas da função intensidade, da função intensidade acumulada, da probabilidade e da probabilidade acumulada em cada um dos 10 semestres analisados por período Noturno.

\begin{tabular}{rrrrr}
\hline \multicolumn{5}{c}{ Noturno } \\
$l$ & $h(l)$ & $H(l)$ & $\zeta(l)$ & Acumulada \\
\hline 1 & 0,0384 & 0,0384 & 0,0384 & 0,0384 \\
2 & 0,0483 & 0,0868 & 0,0465 & 0,0849 \\
3 & 0,0195 & 0,1063 & 0,0179 & 0,1028 \\
4 & 0,0162 & 0,1225 & 0,0145 & 0,1173 \\
5 & 0,0164 & 0,1389 & 0,0145 & 0,1318 \\
6 & 0,0114 & 0,1503 & 0,0099 & 0,1417 \\
7 & 0,0114 & 0,1617 & 0,0098 & 0,1515 \\
8 & 0,0085 & 0,1702 & 0,0072 & 0,1587 \\
9 & 0,0077 & 0,1779 & 0,0065 & 0,1652 \\
10 & 0,0053 & 0,1832 & 0,0045 & 0,1696 \\
\hline
\end{tabular}

\subsubsection{Comparação entre áreas}

Nesta seção comparamos a evasão entre as áreas biológicas, humanas e exatas. Nas Tabelas 7.20 e 7.21 temos as estimativas da função intensidade, da função intensidade acumulada e da 

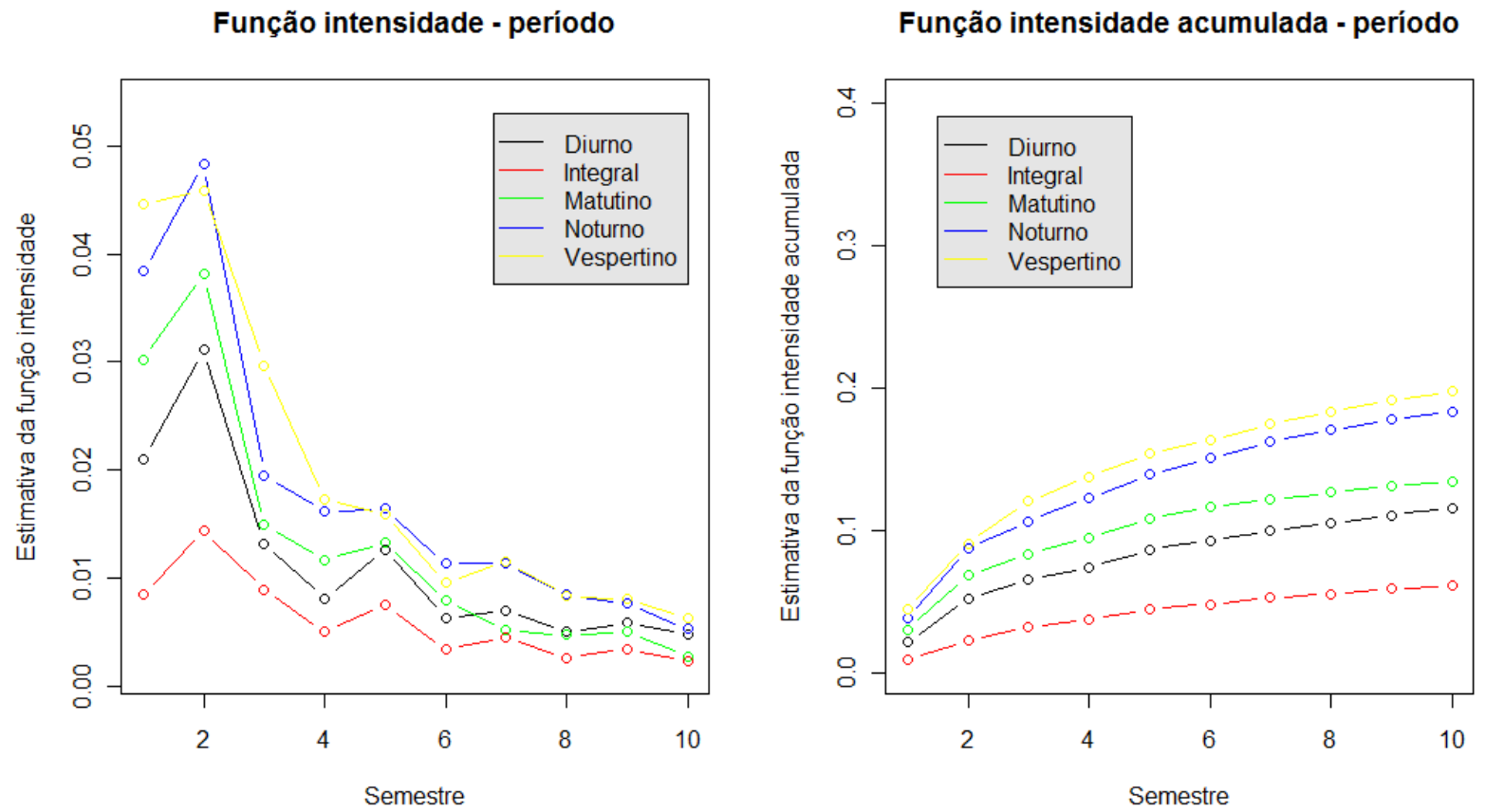

Figura 7.11: Estimadores da função intensidade e da função intensidade acumualada por semestres - período.

Tabela 7.19: Teste de comparação entre períodos.

\begin{tabular}{lrr}
\hline Período & Estatística & Valor descritivo do teste \\
\hline Diurno/Integral & 14,41 & $4,44 \mathrm{E}-47$ \\
Diurno/Matutino & $-3,81$ & 0,00014177 \\
Diurno/Noturno & $-15,90$ & $6,57 \mathrm{E}-57$ \\
Diurno/Vespertino & $-11,03$ & $2,68 \mathrm{E}-28$ \\
Integral/Matutino & $-17,53$ & $8,98 \mathrm{E}-69$ \\
Integral/Noturno & $-42,94$ & $0,00 \mathrm{E}+00$ \\
Integral/Vespertino & $-20,19$ & $1,12 \mathrm{E}-90$ \\
Matutino/Noturno & $-10,18$ & $2,41 \mathrm{E}-24$ \\
Matutino/Vespertino & $-8,02$ & $1,06 \mathrm{E}-15$ \\
Noturno/Vespertino & $-2,04$ & $4,17 \mathrm{E}-02$ \\
\hline
\end{tabular}

distribuição de probabilidade para cada um dos 10 semestres. 
Tabela 7.20: Estimativas da função intensidade, da função intensidade acumulada, da probabilidade e da probabilidade acumulada em cada um dos 10 semestres analisados por área Biológicas, humanas.

\begin{tabular}{rrrrrrrrr}
\hline \multicolumn{1}{c}{ Biológicas } & & \multicolumn{4}{c}{ Humanas } \\
Semestre & $h(l)$ & $H(l)$ & $\zeta(l)$ & Acumulada & $h(l)$ & $H(l)$ & $\zeta(l)$ & Acumulada \\
\hline 1 & 0,00795 & 0,00795 & 0,00795 & 0,00795 & 0,03588 & 0,03588 & 0,03588 & 0,03588 \\
2 & 0,01214 & 0,02009 & 0,01204 & 0,01999 & 0,03979 & 0,07566 & 0,03836 & 0,07423 \\
3 & 0,00750 & 0,02758 & 0,00735 & 0,02734 & 0,01700 & 0,09267 & 0,01574 & 0,08998 \\
4 & 0,00444 & 0,03202 & 0,00432 & 0,03166 & 0,01382 & 0,10649 & 0,01258 & 0,10256 \\
5 & 0,00615 & 0,03818 & 0,00596 & 0,03761 & 0,01448 & 0,12097 & 0,01299 & 0,11555 \\
6 & 0,00256 & 0,04074 & 0,00246 & 0,04008 & 0,00881 & 0,12978 & 0,00780 & 0,12335 \\
7 & 0,00338 & 0,04411 & 0,00324 & 0,04332 & 0,00890 & 0,13868 & 0,00780 & 0,13114 \\
8 & 0,00152 & 0,04564 & 0,00146 & 0,04478 & 0,00691 & 0,14559 & 0,00600 & 0,13715 \\
9 & 0,00234 & 0,04798 & 0,00224 & 0,04701 & 0,00701 & 0,15260 & 0,00605 & 0,14320 \\
10 & 0,00196 & 0,04994 & 0,00187 & 0,04888 & 0,00503 & 0,15763 & 0,00431 & 0,14751 \\
\hline
\end{tabular}

Tabela 7.21: Estimativas da função intensidade, da função intensidade acumulada, da probabilidade e da probabilidade acumulada em cada um dos 10 semestres analisados por área Exatas

\begin{tabular}{rrrrr}
\hline \multicolumn{5}{c}{$\underline{\text { Exatas }}$} \\
Semestre & $h(l)$ & $H(l)$ & $\zeta(l)$ & Acumulada \\
\hline 1 & 0,02116 & 0,02116 & 0,02116 & 0,02116 \\
2 & 0,03711 & 0,05827 & 0,03632 & 0,05748 \\
3 & 0,01744 & 0,07570 & 0,01644 & 0,07392 \\
4 & 0,01065 & 0,08635 & 0,00986 & 0,08378 \\
5 & 0,01370 & 0,10005 & 0,01255 & 0,09633 \\
6 & 0,00889 & 0,10894 & 0,00803 & 0,10436 \\
7 & 0,00922 & 0,11816 & 0,00825 & 0,11262 \\
8 & 0,00653 & 0,12468 & 0,00579 & 0,11841 \\
9 & 0,00606 & 0,13074 & 0,00534 & 0,12375 \\
10 & 0,00363 & 0,13437 & 0,00318 & 0,12693 \\
\hline
\end{tabular}

O interesse é testar as seguintes hipóteses

$$
\left\{\begin{array}{l}
H_{0}: H^{(t)}=H^{(p)} \\
H_{1}: H^{(t)} \neq H^{(p)}
\end{array}\right.
$$



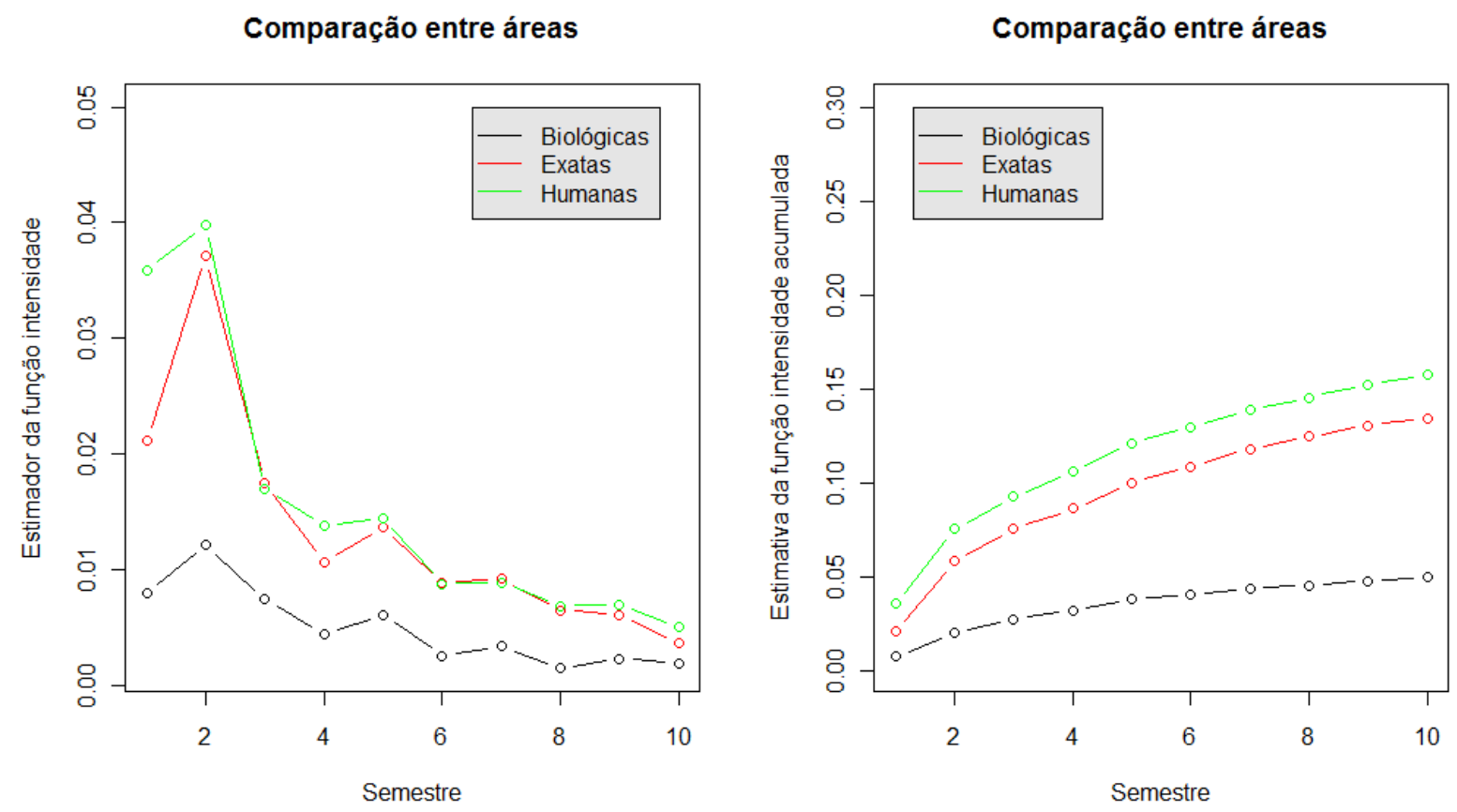

Figura 7.12: Estimadores da função intensidade e da função intensidade acumualada por semestres - área.

Para isso, utilizamos a estatística de teste apresentada nesta seção para comparação entre os anos. Os resultados encontram-se na Tabela 7.22. Utilizando o critério de Bonferroni, adotamos um nível de significância de 0,017. Conluímos que existe diferença significativa entre todas as áreas e que a evasão é maior em humanas, seguido de exatas e por último biológicas. Observando a Figura 7.12, concluímos que a diferença resultante entre humanas e exatas é também conseqüência de uma mudança de comportamento entre os semestres, além de uma queda na evasão. Para humanas a evasão no primeiro semestre é alta sendo pouca a diferença entre esta e a evasão no segundo semestre. Para exatas a evasão no primeiro semestre é bem menor do que no segundo semestre.

Entre biológicas e humanas, e biológicas e exatas a diferença reside especificamente na alta queda da evasão. 
Tabela 7.22: Teste de comparação entre áreas.

\begin{tabular}{lrr}
\hline Área & Estatística & Valor descritivo do teste \\
\hline Biológicas/Exatas & $-30,19$ & $3,55 \mathrm{E}-200$ \\
Biológicas/Humanas & $-42,02$ & $0,00 \mathrm{E}+00$ \\
Exatas/Humanas & $-7,81$ & $5,91 \mathrm{E}-09$ \\
\hline
\end{tabular}

\subsubsection{Portal Evasão}

Ao decorrer deste trabalho desenvolvemos o Portal Evasão. O Portal Evasão, é um sistema interno da USP, onde podemos efetuar análises estatísticas descritivas e específicas a respeito dos alunos de graduação da USP. As análises são efetuadas em linguagem R. O desenvolvimento do sistema ocorreu junto a alunos de pós-graduação da área de Ciências de Computação do ICMC.

No Portal Evasão, até o momento, temos análises básicas como distribuição de freqüência, gráfico de pareto e tabela cruzada, e análises específicas como, tendência da evasão, motivo da evasão e tempo até a conclusão do curso.

O sistema entrou no ar em outubro de 2008 e abaixo temos algumas imagens de sua primeira versão. 


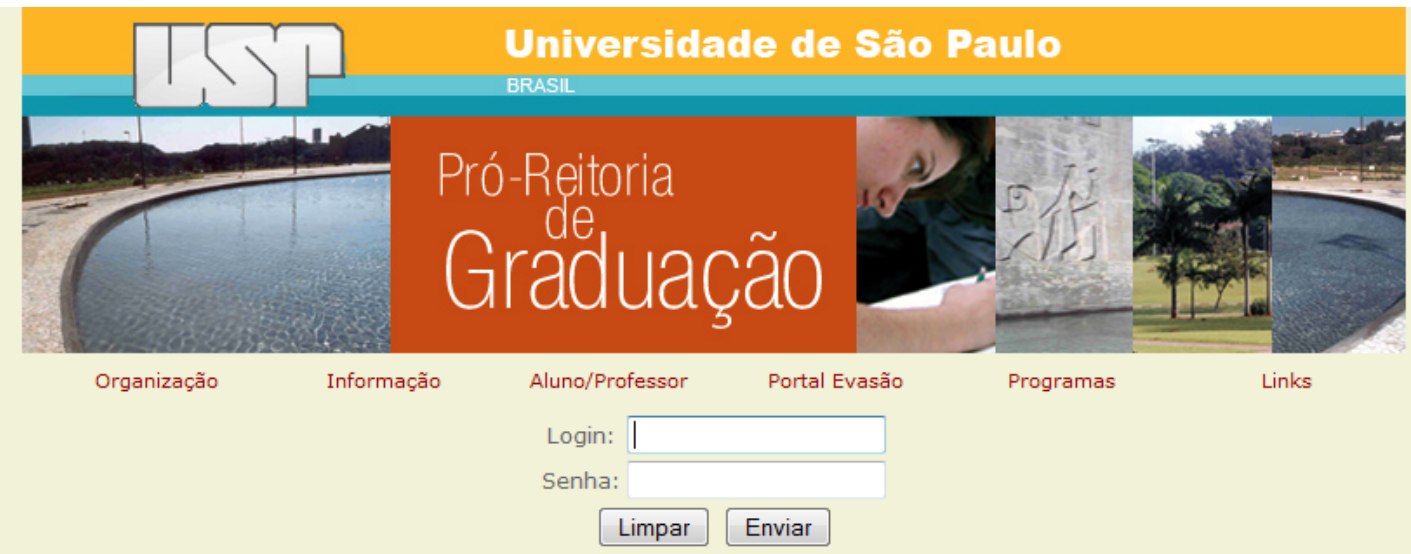

Figura 7.13: Página de login.

Geral

Manual do usuário

Ferramentas Básicas

Ferramentas Especificas

\section{SAEG - Sistema de Análise Estatisticas da Graduação}

::Visão Geral::

\section{Resumo da base de dados (gerada pelo DI em NA)}

Ingressantes por vestibular entre 1999 e 2008 : 87521

Campi: 7

Cursos: 109

Alunos Ativos: 48165
Institutos: 43

Habilitações: 233

Evasões em 2008: 520

Figura 7.14: Página principal. 


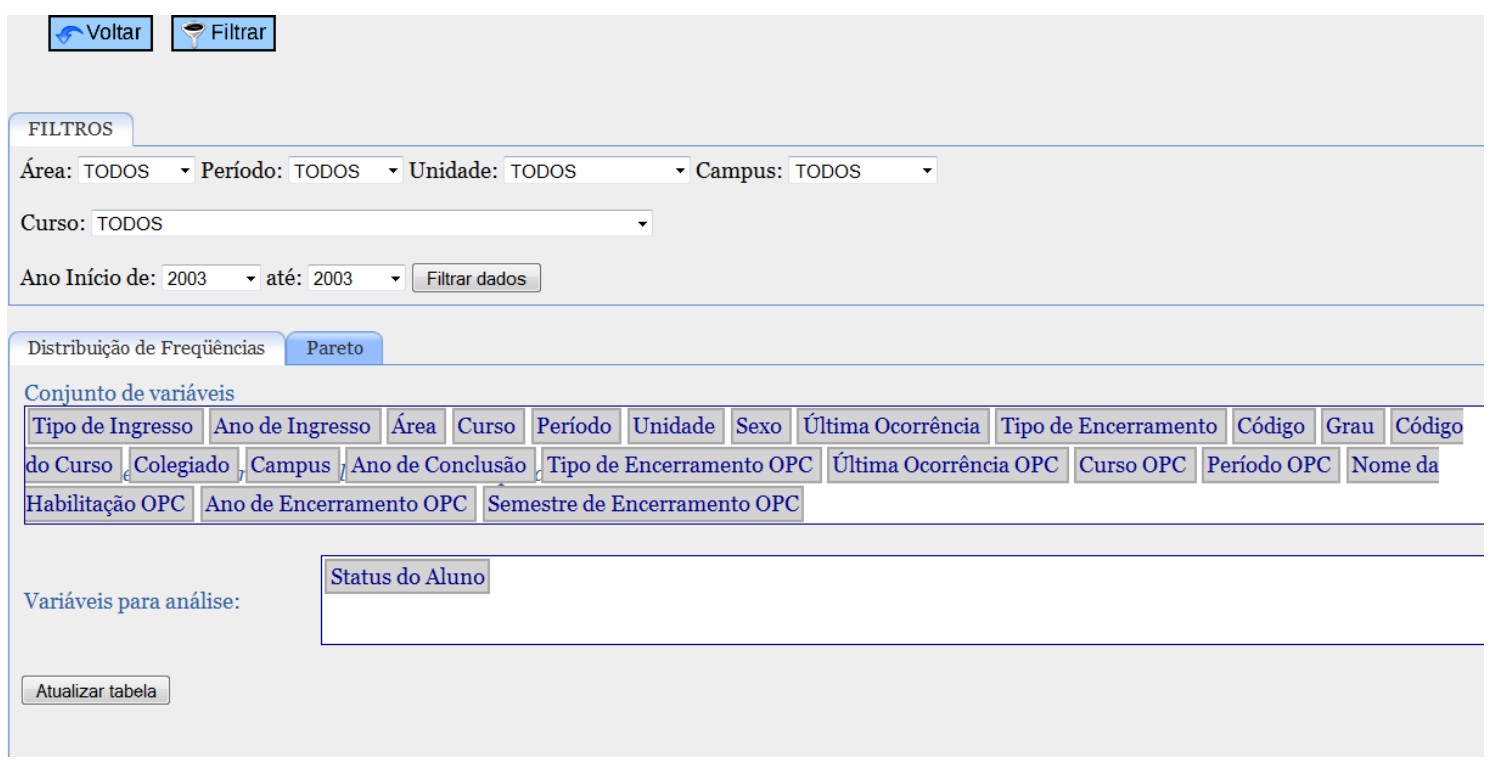

Figura 7.15: Análise de distribuição de freqüência e filtros possiveis de sereme feitos.

Variáveis para análise:

Status do Aluno

Atualizar tabela

Tabela: Distribuição de Frequiências

\begin{tabular}{|rccc|}
\hline Linha & \multicolumn{1}{c}{ Fator } & Freqüência & Porcentagem \\
\hline 1 & Ativos & 48165 & 55.03 \\
\hline 2 & Concluiram & 25983 & 29.69 \\
\hline 3 & Evadiram & 10296 & 11.76 \\
\hline 4 & Faleceram & 59 & 0.07 \\
\hline 5 & Migraram & 2964 & 3.39 \\
\hline 6 & NULL & 54 & 0.06 \\
\hline 7 & Total & 87521 & 100 \\
\hline
\end{tabular}

Figura 7.16: Resultado obtido na distribuição de freqüência.

\subsection{Conclusão}

Analisando os dados para alunos ingressantes entr 2000 e 2004, verificamos que, de forma geral, a evasão vem diminuindo significativamente ao longo dos anos. Concluímos que existe o fator período do curso influência no comportamento da evasão que é maior nos períodos noturno e vespertino (não há diferença entre esse dois), seguido dos períodos matutino, diurno e integral. Comparando os sexos, a evasão é sempre maior entre os homens. A área do curso 
também influência na evasão, e é maior para humanas, seguido de exatas e biológicas. 


\section{Capítulo 8}

\section{Conclusão e Trabalhos Futuros}

Neste trabalho propomos um modelo que é uma extensão do modelo de intensidade multiplicativo desenvolvido por Aalen (1978) e apresentado em Fleming \& Harrington (2005), para variáveis aleatórias multinomiais, com as seguintes características:

- Categorias nulas;

- Razão de chances não proporcionais; e

- Dados na presença de censura.

O modelo sugerido é também adequado para análise de dados multinomiais que atendem às hipóteses necessárias no modelo de regressão multinomial clássico.

Estimamos os parâmetros do modelo utilizando o critério de martingale. Demonstramos que os estimadores da função intensidade, da função intensidade acumulada e da distribuição de probabilidade são estimadores consistentes. Verificamos esse resultados realizando um estudo de simulação.

Propomos testes de hipóteses para comparação dos parâmetros para uma e duas populações. A estatística de teste para comparação de duas populações com dados censurados, apresentada neste trabalho, foi proposta por Mantel (1966) e é baseada na estatística de Mantel-Haenszel para tabelas de contigências estratificadas. Esta estatística ficou conhecida como estatística de logrank e é provavelmente a mais utilizada como estatística de teste para comparação de duas populações com dados censurados. Realizamos estudos de simulação e verificamos que estes 
testes forneceram taxas de não rejeição da hipótese nula bem próximas ao nível de confiança de $95 \%$, para tamanhos amostrais a partir de 80 .

Finalmente, aplicamos o modelo proposto para analisar o comportamento da evasão dos alunos de graduação da Universidade de São Paulo. Analisando os dados para alunos ingressantes entr 2000 e 2004, verificamos que, de forma geral, a evasão vem diminuindo significativamente ao longo dos anos. Concluímos que o fator período do curso influencia no comportamento da evasão e que é maior nos períodos noturno e vespertino (não há diferença entre esse dois), seguido dos períodos matutino, diurno e integral. Comparando os sexos, a evasão é sempre maior entre os homens. A área do curso também influência na evasão e é maior para humanas, seguido de exatas e biológicas.

Como proposta de trabalho futuros, listamos os seguintes itens:

- Extensão da teoria assintótica de integrais estocásticas para diversas populações.

- Extensão da teoria assintótica de teste de hipótese para diversas populações.

- Análise da variância assintótica associada à estatística de logrank.

- Comparação das estatísticas de logrank e Gehan-Wilcoxon sugerida em Fleming \& Harrington (2005) para comparação de diversas populações. 


\section{Apêndice A}

\section{Espaço de Cantor}

Algumas demonstrações associadas à construção do espaço de probabilidade para acomodar uma variável aleatória multinomial.

Lema A.1. A classe $\mathcal{H}$ separa pontos em $S^{\infty}$, ou seja, dados $x$ e y dois pontos distintos pertencentes a $S^{\infty}$ existe um conjunto $\Lambda \in \mathcal{H}$ tal que

$$
\mathbb{1}_{\{\Lambda\}}(x) \neq \mathbb{1}_{\{\Lambda\}}(y) .
$$

Demonstração: Tomemos dois elementos distintos, $x$ e $y$, em $S^{\infty}$. Temos que esses elementos são da seguinte forma

$$
\begin{aligned}
& x=\left(\alpha_{1}, \alpha_{2}, \ldots, \alpha_{k}, \ldots\right), \quad \alpha_{i}=0 \text { ou } 1 \\
& y=\left(\beta_{1}, \beta_{2}, \ldots, \beta_{k}, \ldots\right), \quad \beta_{i}=0 \text { ou } 1, \quad i=1,2, \ldots, k, \ldots
\end{aligned}
$$

Como $x$ e $y$ são distintos, então $x$ e $y$ diferem em pelo menos uma coordenada, digamos na $j$-ésima coordenada. Assim, $\alpha_{j} \neq \beta_{j}$ e, conseqüentemente,

$$
\pi_{j}^{-1}\left(\left\{\alpha_{j}\right\}\right) \neq \pi_{j}^{-1}\left(\left\{\beta_{j}\right\}\right) .
$$

Pelo resultado 1 ,

$$
\pi_{j}^{-1}\left(\left\{\alpha_{j}\right\}\right) \cap \pi_{j}^{-1}\left(\left\{\beta_{j}\right\}\right)=\emptyset .
$$

Desta forma, basta tomarmos $\Lambda=\pi_{j}^{-1}\left(\left\{\alpha_{j}\right\}\right)$ para termos

$$
\mathbb{1}_{\{\Lambda\}}(x)=1 \neq \mathbb{1}_{\{\Lambda\}}(y)=0 .
$$


Lema A.2. A classe $\mathcal{H}$ de subconjuntos de $S^{\infty}$ é compacta, isto é, para toda seqüência $\left\{C_{n}\right\} \in$ $\mathcal{H}$ tal que $\bigcap_{n=1}^{\infty} C_{n}=\emptyset$, existe $n_{0} \in \mathbb{N}$ tal que

$$
\bigcap_{n=1}^{n_{0}} C_{i}=\emptyset
$$

Demonstração: Consideremos a seqüência $\left\{C_{n}\right\}_{n} \in \mathcal{H}$ tal que

$$
\bigcap_{n=1}^{\infty} C_{n}=0 .
$$

Assim, existem pelo menos dois elementos da seqüência $\left\{C_{n}\right\}_{n}$ que diferem em pelo menos uma coordenada. Ou seja, existem $r, s \in \mathbb{N}$ tais que

$$
C_{r}=\pi_{k}^{-1}(\{1\}) \text { e } C_{s}=\pi_{k}^{-1}(\{0\})
$$

para algum $k \in \mathbb{N}$. Tomemos $n_{0}=\max \{r, s\}$. Assim,

$$
\bigcap_{n=1}^{n_{0}} C_{n}=\emptyset
$$

$\Lambda$

Lema A.3. A classe $\Delta$ separa pontos em $S^{\infty}$.

Demonstração: Sejam $A$ e $B$ elementos distintos de $\Delta$. Ou seja,

$$
A=\pi_{v_{1}^{A}}^{-1}\left(\left\{\omega_{v_{1}^{A}}\right\}\right) \cap \ldots \cap \pi_{v_{n}^{A}}^{-1}\left(\left\{\omega_{v_{n}^{A}}\right\}\right)
$$

e

$$
B=\pi_{v_{1}^{B}}^{-1}\left(\left\{\omega_{v_{1}^{B}}\right\}\right) \cap \ldots \cap \pi_{v_{n}^{B}}^{-1}\left(\left\{\omega_{v_{n}^{B}}\right\}\right)
$$

$\operatorname{com} \pi_{v_{k}^{A}}^{-1}\left(\left\{\omega_{v_{k}^{A}}\right\}\right) \neq \pi_{v_{k}^{B}}^{-1}\left(\left\{\omega_{v_{k}^{B}}\right\}\right)$ para algum $k \in \mathbb{N}$. Como já vimos, $\mathcal{H}$ separa pontos. Logo, existe $\Lambda \in \mathcal{H}$ tal que

$$
\pi_{v_{k}^{A}}^{-1}\left(\left\{\omega_{v_{k}^{A}}\right\}\right) \in \Lambda \text { e } \pi_{v_{k}^{B}}^{-1}\left(\left\{\omega_{v_{k}^{B}}\right\}\right) \in ? ? \Lambda .
$$

Assim, $A \in \Lambda$ e $B \in$ ?? $\Lambda$. Mais do que isso, existe $\Lambda_{1} \subset \Delta\left(\Lambda_{1} \subset \Lambda\right)$ tal que $A \in \Lambda_{1}$, mas $B \in ? ? ? \Lambda_{1}$. Portanto, $\Delta$ separa pontos. 
Lema A.4. $\Delta$ é uma classe compacta.

Demonstração: Seja $\left\{\Delta_{n}\right\}_{n} \subset \Delta$ uma seqüência de conjuntos tais que

$$
\bigcap_{n=1}^{\infty} \Delta_{n}=\emptyset
$$

sendo

$$
\Delta_{n}=\pi_{v_{1}^{n}}^{-1}\left(\left\{\omega_{v_{1}^{n}}\right\}\right) \cap \ldots \cap \pi_{v_{k_{n}}^{n}}^{-1}\left(\left\{\omega_{v_{k_{n}}^{n}}\right\}\right), \quad \pi_{v_{i}^{n}}^{-1}\left(\left\{\omega_{v_{i}^{n}}\right\}\right) \in \mathcal{H}, \quad i=1, \ldots, k_{n}, \quad n \in \mathbb{N} .
$$

Assim,

$$
\bigcap_{n=1}^{\infty} \Delta_{n}=\bigcap_{n=1}^{\infty}\left(\bigcap_{i=1}^{k_{n}} \pi_{v_{i}^{n}}^{-1}\left(\left\{\omega_{v_{i}^{n}}\right\}\right)\right) .
$$

Com isso, $\bigcap_{n=1}^{\infty} \Delta_{n}$ é interseção enumerável de elementos de $\mathcal{H}$. Portanto, podemos escrever esta interseção como

$$
\bigcap_{n=1}^{\infty} \Delta_{n}=\bigcap_{n=1}^{\infty} C_{n}, \quad C_{n} \in \mathcal{H} .
$$

Como $\mathcal{H}$ é classe compacta, existe $n_{0} \in \mathbb{N}$ tal que

$$
\bigcap_{n=1}^{n_{0}} \Delta_{n}=\bigcap_{n=1}^{n_{0}} C_{n}=\emptyset \text {. }
$$

Proposição A.1. A classe $\mathcal{C}$ é compacta.

Demonstração: Consideremos em $\mathcal{C}$ uma seqüência de conjuntos $\left\{C_{n}\right\}_{n \in \mathbb{N}}$ com a p.f.i. Para provar que $\mathcal{C}$ é uma classe compacta devemos verificar que $\bigcap_{i=1}^{\infty} C_{i} \neq \emptyset$. Agora, pela definição da classe $\mathcal{C}$ temos que

$$
C_{j}=A_{j 1} \cup A_{j 2} \cup \ldots \cup A_{j k_{j}}, \quad \forall j \text { e } A_{j_{s}} \in \Delta, \quad s=1, \ldots, k_{j} .
$$

Como $\Delta$ é classe compacta, basta obter uma seqüênia $A_{1 i_{1}}, A_{2 i_{2}}, \ldots$ com a p.i.f., porque $\bigcap_{j=1}^{\infty} C_{j i_{j}} \subseteq \bigcap_{j=1}^{\infty} A_{j}$. A partir do Lema ?? obtemos esta seqüência, construindo-a indutivamente:

$$
C_{1 i_{1}}, C_{2 i_{2}}, \ldots, C_{n i_{n}}, A_{n+1}, A_{n+2}
$$




\section{Referências Bibliográficas}

Aalen, O. O. (1978). Nonparametric Inference for a Family of Counting Processes. Annals of Statistics, 6, 701-726.

Begg, C. \& Gray, R. (1984). Calculations of polychotomous logistic regression estimates using individualized regressions. Biometrika, 71, 1-18.

Fleming, T. \& Harrington, D. (2005). Counting Processes and Survival Analysis. Wiley, New Jersey.

Helland, I. S. (1982). Central Limit Theorems for Martingales with Discrete or Continuous Time. Scand J Statist, , 9, 79-94.

Hosmer, D. \& Lemeshow, S. (1989). Applied Logistic Regression. Wiley, New york, first edition.

Leao, D., Fragoso, M., Val, J. \& Andrade, M. (2005). Separable Hausdorff Measurable Randon Spaces. Statistics e Probability Letters, , 71, 347-359.

Mantel, N. (1966). Evaluation of survival data and two new rank order statistics arising in its consideration. Cancer Chemother, 50, 163-70.

McCullagh, P. \& Nelder, J. A. (1989). Generalized Linear Models. Chapman \& Hall, London, second edition.

Rom, M. \& Cohen, A. (1995). Estimation in the polytomous logistic regression model. Jornal of Statistical Planning and Inference., 43, 341-353.

Shiryayev, A. (1984). Probability. Springer-Verlag, New York.

Stout, W. (1970). Almost sure Convergence. Academic Press, Inc.(London) Ltd., New York. 